\title{
The computational architecture of the human auditory cortex
}

Citation for published version (APA):

Santoro, R. (2015). The computational architecture of the human auditory cortex. [Doctoral Thesis, Maastricht University]. Maastricht University. https://doi.org/10.26481/dis.20150306rs

Document status and date:

Published: 01/01/2015

DOI:

10.26481/dis.20150306rs

Document Version:

Publisher's PDF, also known as Version of record

\section{Please check the document version of this publication:}

- A submitted manuscript is the version of the article upon submission and before peer-review. There can be important differences between the submitted version and the official published version of record.

People interested in the research are advised to contact the author for the final version of the publication, or visit the DOI to the publisher's website.

- The final author version and the galley proof are versions of the publication after peer review.

- The final published version features the final layout of the paper including the volume, issue and page numbers.

Link to publication

\footnotetext{
General rights rights.

- You may freely distribute the URL identifying the publication in the public portal. please follow below link for the End User Agreement:

www.umlib.nl/taverne-license

Take down policy

If you believe that this document breaches copyright please contact us at:

repository@maastrichtuniversity.nl

providing details and we will investigate your claim.
}

Copyright and moral rights for the publications made accessible in the public portal are retained by the authors and/or other copyright owners and it is a condition of accessing publications that users recognise and abide by the legal requirements associated with these

- Users may download and print one copy of any publication from the public portal for the purpose of private study or research.

- You may not further distribute the material or use it for any profit-making activity or commercial gain

If the publication is distributed under the terms of Article $25 \mathrm{fa}$ of the Dutch Copyright Act, indicated by the "Taverne" license above, 
The Computational Architecture of the Human Auditory Cortex

\author{
Roberta Santoro
}


(c) Roberta Santoro, Maastricht 2015.

All rights reserved. No part of this publication may be reproduced, stored in a retrieval system or transmitted in any form or by any means, electronic, mechanical, photocopying, recording or otherwise, without prior written permission of the publisher.

Printed by CPI-Wöhrmann Print Service - Zutphen

ISBN 978-94-6203-747-2 


\title{
The Computational Architecture of the Human
}

\author{
Auditory Cortex
}

\section{DISSERTATION}

to obtain the degree of Doctor at the Maastricht University, on the authority of the Rector Magnificus, Prof.dr. L.L.G Soete in accordance with the decision of the Board of Deans, to be defended in public on Friday $6^{\text {th }}$ March 2015 at 10:00 hours

by

Roberta Santoro 
Supervisor

Prof. dr. Elia Formisano

Co-supervisor

Dr. Federico De Martino

Dr. Michelle Moerel (University of Minnesota, USA)

\section{Assessment Committee}

Prof. dr. Bernadette M Jansma (Chair)

Prof. dr. Fabrizio Esposito (University of Salerno, Italy)

Prof. dr. Jack Gallant (University of California, Berkeley, USA)

Dr. Kâmil Uludağ 


\section{Contents}

1 General Introduction $\quad 7$

2 Encoding of Natural Sounds at Multiple Spectral and 25 Temporal Resolutions in the Human Auditory Cortex

3 Spectral and temporal properties of auditory cortical 69 regions and their relation to the acoustic discriminability of natural sounds

4 Re-synthesis of real-life sounds from non-invasively measured auditory cortical response patterns

$5 \quad$ Summary and Conclusions

6 Knowledge valorization

Acknowledgements

Publications

Curriculum Vitae 

Chapter 1

General Introduction 


\section{Introduction}

7 AM. A disturbing ringing tone goes off: it is time to start the day. The sound of water running in the shower tells you that somebody was faster than you. When you knock on the door of the bathroom, Walt's usual "First come, first serve!" makes you smile. You switch on the radio and your favorite song is playing.

This realistic scenario illustrates how sounds are an integral part of our daily lives. We are constantly exposed to sounds from widely disparate sources and, more importantly, our brain is able to use these sounds to accomplish remarkably complex tasks, such as recognizing somebody's voice, understanding speech, enjoying music or locating the source of a sound. How can our brain extract such a rich amount of information based on the sounds that we hear? Before we can answer the question of how high-level auditory perceptual tasks are accomplished, we need to understand the nature of the low-level sensory representation of incoming sounds (i.e. which acoustic dimensions - or features - are encoded). One can think of the human auditory system as a sophisticated engineering system that takes sounds as input and generates auditory percepts as output. Engineering systems that perform operations on complex inputs rely on a front end that receives the input in its original form and transforms it into a signal that is better suited for generating the final output. Similarly, the auditory system must transform incoming sounds into representations that form the basis of auditory perception. While much is known about the early stages of this transformation at the auditory periphery, the representational mechanisms of incoming sounds at the later, cortical stages are less well understood. The research described in this thesis aims at extending our knowledge of how complex, natural sounds are encoded within the human auditory cortex.

Much of our knowledge about the cortical representation of the acoustic structure of incoming sounds is based on brain responses to simple synthetic stimuli (e.g. tones or noise bursts). However, natural sounds, i.e. sounds that we encounter in our everyday life, are acoustically much more complex and are characterized by a degree of behavioral relevance and familiarity that is not achievable with synthetic stimuli. As a consequence, cortical responses to simple, artificial stimuli differ from responses elicited by natural sounds (Wang et al., 1995; Theunissen et al., 2000; Bitterman et al., 2008; Laudanski et al., 2012; Theunissen and Elie, 2014). Thus, although highly controllable, simple artificial sounds may not be appropriate for investigating the representational mechanisms involved in the processing of behaviorally relevant sounds. On the other hand, the majority of 
studies that have employed natural sounds have focused on higher processing levels, revealing the contribution of specific regions (e.g. Belin et al., 2000; Lewis et al., 2005; Ahveninen et al., 2006; Altmann et al., 2007) and networks (Formisano et al., 2008; Staeren et al., 2009; Bonte et al., 2014) within the auditory cortex to the representation of semantic/categorical and behaviorally relevant information. But which acoustic features the auditory cortex uses to derive these higher-level representations remains poorly understood.

This thesis combines functional magnetic resonance imaging (fMRI) and computational modeling to investigate how the human auditory cortex encodes complex, ecologically valid sounds. More specifically, the study presented in Chapter 2 compares competing models of auditory representations and explores the topography of voxels' sensitivity to spectral and temporal sound modulations. In Chapter 3, we employ model-based decoding techniques to further investigate the contribution of distinct auditory cortical regions to the sensory encoding of natural sounds. Finally, Chapter 4 implements model-based stimulus reconstruction to re-synthesize arbitrary auditory stimuli based on the fMRI response patterns that they elicit.

The present chapter provides the background for the studies reported in this thesis. First, spectral and temporal modulations are introduced, and previous findings related to their behavioral relevance and cortical representation are reviewed. Then, a description of the signal acquisition and analysis methods employed is provided.

\section{Sound representation at the auditory periphery: spectral and temporal modulations}

The first crucial transformation of incoming sounds takes place at the auditory periphery (from the outer ear until the cochlear nerve) (Goldstein, 2014). Sounds arrive at the ears as pressure waves that propagate through the air. After entering the outer ear, sound waves are transmitted through the middle ear to induce mechanical vibrations in the basilar membrane within the cochlea of the inner ear. Because the stiffness of the basilar membrane changes progressively along its length, the place of its maximum displacement depends on the frequency of the incoming sound. As a result, the different frequency components of a sound are spatially segregated within the cochlea, with high frequencies represented near the base, and low frequencies near the apex. This ordered frequency-to-place mapping is referred to as tonotopy and represents a fundamental organizational 
principle throughout the auditory system, where frequency-selective responses are ubiquitous (Schreiner et al., 2011).

The auditory periphery transforms the input sound from its one-dimensional temporal representation (sound wave) to a two-dimensional time-frequency representation (Figure 1.1). This spectrogram-like representation describes the time varying amplitude of each acoustic frequency and is better suited for highlighting the rich and dynamic spectral structure of natural sounds. In the timefrequency space, natural sounds are characterized by peaks and valleys of amplitude along both the temporal and spectral dimension (Figure 1.2). These amplitude oscillations are referred to as modulations and are not specific to a given temporal or spectral scale, but rather occur over multiple scales (Singh and Theunissen, 2003). For example, in the spectrogram of a speech sample, formants occur on a larger spectral scale (i.e. lower modulation frequency) than the harmonics of the speaker's fundamental frequency. Likewise, temporal modulations at different time scales co-occur; for example, modulations associated with syllabic events are characterized by a longer temporal scale than modulations associated with phonemes transitions (Rosen, 1992).

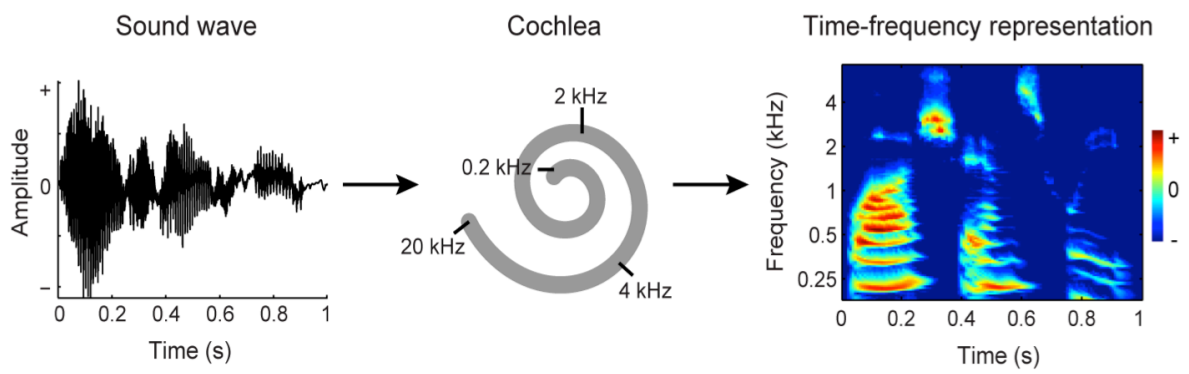

Figure 1.1. Signal transformation performed by the cochlea. Sounds enter our ears as one-dimensional pressure waveforms (left; in the example, the words "Wash and sweep" are uttered by a male speaker). Within the cochlea, the different frequency components of a sound wave are spatially segregated (middle). This results into a time-frequency representation, which depicts the frequency content of the incoming sound and how it changes over time (right; the color code indicates the amplitude of each frequency component at any given time).

Evidence from physiological and psychoacoustic investigations suggests that, in addition to frequency, the cortical representation of natural sounds entails the explicit encoding of their spectral and temporal modulations through dedicated 
modulation-detectors. The following paragraphs provide an overview of the main findings that support this hypothesis.

A

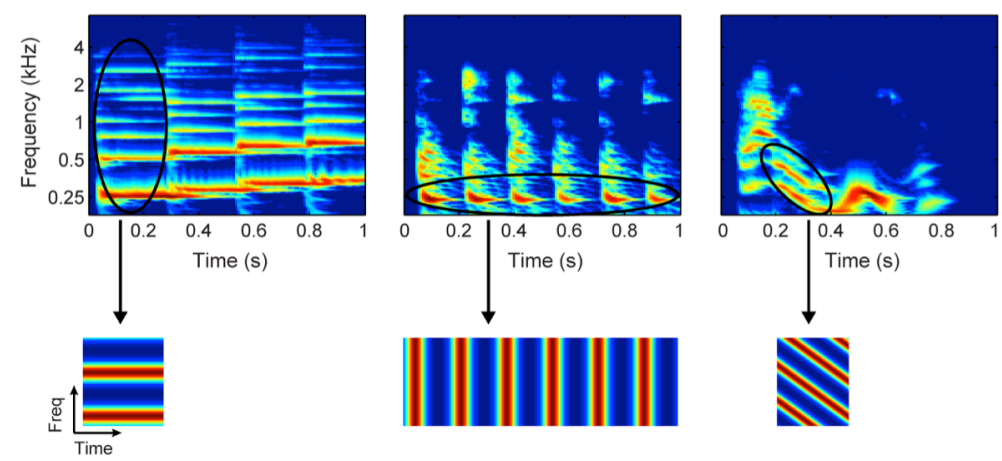

B

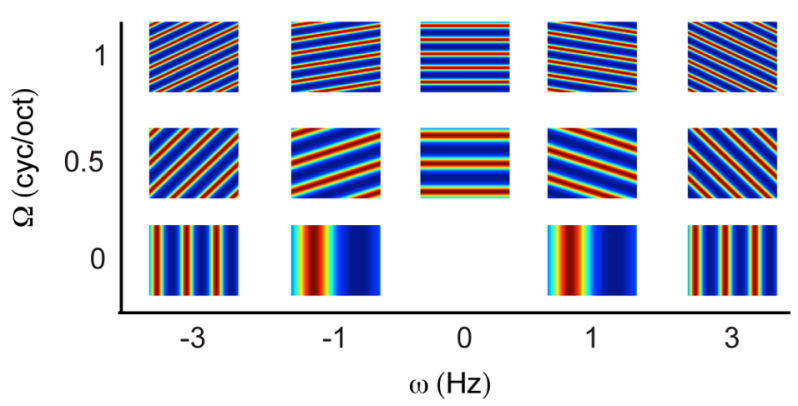

Figure 1.2. Schematic of spectral and temporal modulations. (A) Spectrogram of three exemplary natural sounds used as stimuli in the studies presented in this thesis. Spectrograms are computed using a computational model mimicking early auditory processing (Chi et al., 2005). Black ellipses highlight examples of spectral (left), temporal (middle), and spectro-temporal (right) modulations. Schematics of these modulation patterns are shown under the spectrograms. Spectral modulations are amplitude oscillations along the frequency axis (e.g. speech formants), while temporal modulations are oscillations along the temporal axis (e.g. syllabic structure in speech). Spectro-temporal modulations are combined oscillations over both time and frequency (e.g. formant transitions). (B) Spectral and temporal modulations are defined by the frequency of the oscillation, which determines the spacing between consecutive amplitude peaks. Schematic spectrograms illustrate how the modulation pattern changes with spectral $(\Omega)$ and temporal $(\omega)$ modulation frequencies. Purely temporal and spectral modulations are defined for $\Omega=0$ and $\omega$ $=0$, respectively. By convention, positive and negative temporal modulation frequencies distinguish between downward and upward spectro-temporal modulations. Artificial sounds whose spectrogram resembles the spectral and spectro-temporal modulations depicted here are referred to as static and dynamic ripples, respectively. 


\section{Behavioral relevance of spectral and temporal modulations}

Psychoacoustic studies have investigated human sensitivity to spectral (Green, 1986; Eddins and Bero, 2007) and temporal modulations (Viemeister, 1979; Bacon and Viemeister, 1985; O'Connor et al., 2011), as well as combined spectrotemporal modulations (Chi et al., 1999). These studies implemented a modulation detection (or discrimination) task where the modulation depth (i.e. the peak-tovalley distance) is manipulated in order to derive the listener's modulation detection threshold, that is the minimum modulation depth required to detect changes in the spectrogram amplitude. Detection thresholds are measured over a range of modulation frequencies to yield a modulation transfer function (MTF). Measurements of modulation detection thresholds show that normal-hearing listeners are most sensitive to spectral and temporal modulations at low modulation frequencies. The exact cutoff modulation frequency of this low-pass profile depends on the stimulus type and it ranges between $10-64 \mathrm{~Hz}$ for temporal modulations and $2-4$ cyc/oct for spectral modulations (Chi et al., 1999).

Evidence of the behavioral relevance of spectral and temporal modulations has come from psychoacoustic investigations that show how performance of auditory perceptual tasks correlates with a systematic degradation of the stimulus. A common approach of these studies is to measure task performance in relation to stimuli that have been filtered to remove spectral and/or temporal modulations at specific frequencies. This results in a task-specific MTF that indicates which modulation frequencies are most relevant for the task of interest. When applied to speech intelligibility, this method has revealed that speech comprehension is significantly impaired when low-frequency spectral or temporal modulations are removed (Drullman et al., 1994; Shannon et al., 1995; Liu and Eddins, 2008; Elliott and Theunissen, 2009). Specifically, the MTF for speech intelligibility was shown to be band-pass for temporal modulations and low-pass for spectral modulations, with temporal modulations between 1 and $7 \mathrm{~Hz}$ and spectral modulations $<1$ $\mathrm{cyc} / \mathrm{kHz}$ being the most relevant for maintaining speech comprehension (Elliott and Theunissen, 2009).

\section{Cortical processing of spectral and temporal modulations}

Numerous psychoacoustical and physiological studies have investigated the mechanisms underlying modulation perception by focusing on temporal and spectral modulations alone, or the combination of the two (spectro-temporal modulations). Examples of stimuli that are typically used are amplitude-modulated 
noise or tones for temporal modulations, static ripples for spectral modulations, and dynamic ripples for spectro-temporal modulations (Figure 1.2B). Results from behavioral experiments on selective adaptation (Tansley, 1983), modulation masking (Bacon, 1989; Houtgast, 1989; Ewert and Dau, 2000; Saoji and Eddins, 2007) and perceptual learning (Sabin et al., 2012) suggest that human modulation perception is mediated by a bank of modulation-selective channels, each tuned to a given modulation frequency. Just as the cochlea decomposes the acoustic waveform into its frequency components, the auditory cortex is postulated to decompose the spectrogram into its modulation components via arrays of filters that are tuned to different spectral and temporal modulation frequencies. Channels tuned to low modulation frequencies extract a smooth version of the spectrogram, while channels tuned to high modulation frequencies capture its fine-grained spectral and temporal amplitude variations. Such decomposition into modulation components is analogous to the spatial frequency analysis of incoming images that is performed in the low-level visual cortex (Versnel et al., 1995). Evidence for the filter bank model of modulation processing has come from electrophysiological recordings in animals, which have shown that neurons in the (primary) auditory cortex are tuned to different spectral (e.g. Schreiner and Calhoun, 1994; Shamma et al., 1995; Versnel et al., 1995; Versnel and Shamma, 1998), temporal (see Joris et al., 2004 for a review) and spectro-temporal modulation frequencies (e.g. Kowalski et al., 1996; deCharms, 1998; Depireux et al., 2001). In humans, several studies using simple artificial stimuli have confirmed cortical tuning to modulation frequency (Giraud et al., 2000; Langers et al., 2003; Liégeois-Chauvel et al., 2004; Schönwiesner and Zatorre, 2009; Warrier et al., 2009; Samson et al., 2011; Barton and Venezia, 2012; Overath et al., 2012; Herdener et al., 2013). To date, it is not clear how spectral and temporal modulation tuning relate to each other, as well as to the frequency-selective responses observed throughout the auditory cortex. This issue is addressed in Chapter 2 of this thesis.

A great effort has been made to determine the spatial distribution of modulation tuning properties within and across cortical regions. Topographic maps of stimulus physical dimensions are a well-established organizational principle of the auditory cortex and it has been argued that an ordered spatial distribution of modulation frequencies might exist (Schreiner and Winer, 2007). However, while previous studies found clusters of neuronal populations responding better to low or high modulation frequencies, reports of a topographical representation of modulation frequency preference have not been consistent (for temporal modulations, see e.g. Giraud et al., 2000; Barton and Venezia, 2012; Herdener et 
al., 2013; for spectro-temporal modulations, see Langers et al., 2003; Schönwiesner and Zatorre, 2009). Parallel to the hypothesis of intra-hemispheric topographic maps of modulation frequencies, it has been suggested that a trade-off exists between the hemispheres, with the left and right hemispheres especially suited for the processing of high-frequency temporal and spectral modulations respectively (Zatorre and Belin, 2001; Schönwiesner et al., 2005). To date, the spatial layout of spectral and temporal modulation tuning properties across the auditory cortex remains elusive. The studies presented in Chapters 2 and 3 address this open question.

\section{Investigating the cortical processing of natural sounds}

In the present thesis, the cortical representation of natural sounds in terms of their spectral and temporal modulations is investigated using fMRI. FMRI is a noninvasive neuroimaging technique that provides an indirect measure of neuronal activity (e.g. Logothetis et al., 2001). When neurons are activated by a stimulus or a cognitive task, their metabolic demands increase. This leads to a modulation of the ratio between oxygenated and deoxygenated hemoglobin that is picked up by the fMRI signal, therefore known as blood-oxygen-level dependent (BOLD) hemodynamic response (Ogawa et al., 1992).

Because of its relatively high spatial resolution and extensive brain coverage, fMRI has been extensively used to investigate the sensory representation of sounds in the human auditory cortex. The traditional approach consists in translating research hypotheses into specific stimulus types: a given acoustic feature is posited to be encoded by the auditory cortex and brain activity is measured in response to synthetic stimuli that vary with respect to the feature of interest. For example, cortical tuning to spectral and temporal modulations has been investigated by using e.g. (static and dynamic) ripples and amplitude modulated white noise. As stated at the beginning of this chapter, the main limitation of this approach that uses artificial stimuli lies in its poor ecological validity. Recently, an alternative method has been introduced that allows overcoming this limitation. This new approach had previously been established in electrophysiology and is known under several names, among which are system identification (Wu et al., 2006), encoding (Naselaris et al., 2010), and model-based fMRI (O'Doherty et al., 2007). Contrary to the traditional approach, in system identification the hypothesis about the encoded acoustic features is formalized as a computational model of auditory processing, rather than made explicit in the stimuli (Figure 1.3). In other words, the auditory 
system is probed with natural sounds and the computational model provides a quantitative, explicit description of the way input stimuli are transformed and represented in the $\mathrm{FMRI}$ signal.

\section{Traditional approach}

\section{System identification}

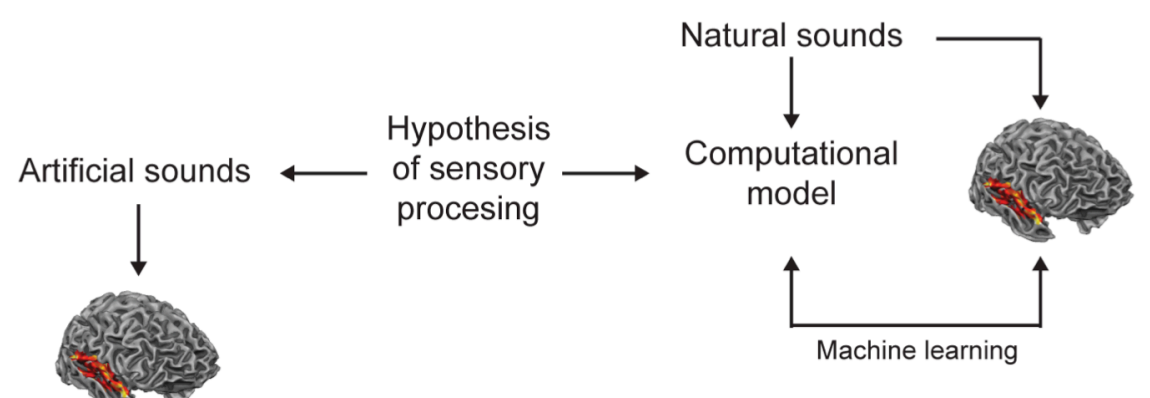

Figure 1.3. Traditional vs system identification approach. In the traditional approach, a hypothesis of sensory processing is translated into artificial sounds that are then used to probe the auditory cortex. Conversely, in the system identification approach, brain activity is measured in response to natural sounds and the hypothesis of sensory processing is quantitatively implemented as a computational model. Machine learning techniques are then used to fit the model against the observed fMRI responses.

A system identification procedure entails the following steps (Naselaris et al., 2010). First, fMRI responses to a large number of natural stimuli are measured. Second, stimuli are processed using a computational model that extracts the features of interest. Subsequently, data are subdivided into independent training and testing sets and machine-learning algorithms are used to estimate an inputoutput functional relationship between the features and the fMRI responses in the training set. Finally, the test set is used to assess the ability of the learned functional relationship to predict data not used during training. Prediction accuracy significantly above chance indicates that the employed computational model is an adequate descriptor of the sensory processing that is reflected in the fMRI signal.

Compared to the traditional approach, system identification has two main advantages. First, it allows investigating auditory sensory processing in an ecological valid setting. Second, alternative hypotheses (i.e. different computational models) can be tested and directly compared using the same dataset. 
System identification can be applied within an encoding or decoding framework. In the encoding approach, an input-output relationship (encoding model) is estimated at each voxel. The voxel's activity is modeled as a linear combination of the features extracted by the model of sensory processing, and the estimated coefficients represent the voxel's sensitivity to these features. Conversely, the decoding approach uses the fMRI signal from multiple voxels to predict individual sensory features. In the present thesis, the multivariate system identification procedure is referred to as model-based feature reconstruction (Chapter 3).

One of the most sophisticated applications of system identification is stimulus reconstruction. The goal of stimulus reconstruction is to synthesize an arbitrary stimulus based on the $\mathrm{fMRI}$ response pattern that it elicits. As such, it constitutes the ultimate proof of our understanding of the sensory input-output transformation. So far, the combination of stimulus reconstruction techniques and fMRI has been restricted to the study of the visual system. In this context, researchers have been able to reconstruct simple geometric patterns and complex natural images in both static (Thirion et al., 2006; Miyawaki et al., 2008; Naselaris et al., 2009; Schoenmakers et al., 2013) and dynamic (Nishimoto et al., 2011) conditions. The study presented in chapter 4 tackles the challenge - so far unexplored - of reconstructing natural sounds based on patterns of $\mathrm{fMRI}$ responses.

\section{A computational model of auditory processing}

The computational model of auditory processing employed in the present thesis describes the processing of three relevant properties of natural sounds: frequency, temporal modulations and spectral modulations. The model has been proposed by (Chi et al., 2005) and is inspired by findings from neurophysiological and psychoacoustical investigations in early and central stages of the mammalian auditory system. The auditory model consists of two main components (Figure 1.4): an early stage that accounts for the transformations that acoustic signals undergo in the early auditory system, from the cochlea to the midbrain; and a cortical stage that simulates the processing of the acoustic input at the level of the (primary) auditory cortex. The spectral analysis performed by the cochlea is mimicked by a bank of 128 overlapping bandpass filters with constant- $Q\left(Q_{10 d B}=3\right)$, equally spaced along a logarithmic frequency axis over a range of 5.3 octaves $(f=180$ $7040 \mathrm{~Hz}$ ). The output of each filter enters a hair cell stage, where it undergoes high-pass filtering, optional non-linear compression and low-pass filtering. A 
midbrain stage models the enhancement of frequency selectivity as a first-order derivative with respect to the frequency axis, followed by a half-wave rectification. Finally, a short-term temporal integration accounts for the loss of phase locking observed in the midbrain. The auditory spectrogram generated by the early stage is further analyzed by the cortical stage, which explicitly encodes the modulation content of the spectrogram. Cortical neurons are modeled as 2-dimensional (2D) modulation selective filters that are tuned to a specific combination of spectral and temporal modulations, and operate over a limited range of frequencies along the tonotopic axis. These filters have constant $\mathrm{Q}$ and are directional, i.e. they respond either to upward or downward frequency sweeps. Computationally, the cortical filter bank performs a complex wavelet decomposition of the auditory spectrogram. The magnitude of such decomposition yields a phase-invariant measure of modulation content. Ultimately, the model's output is a multi-resolution representation of the spectrogram envelope as a function of time, frequency, spectral and temporal modulations, and directionality.

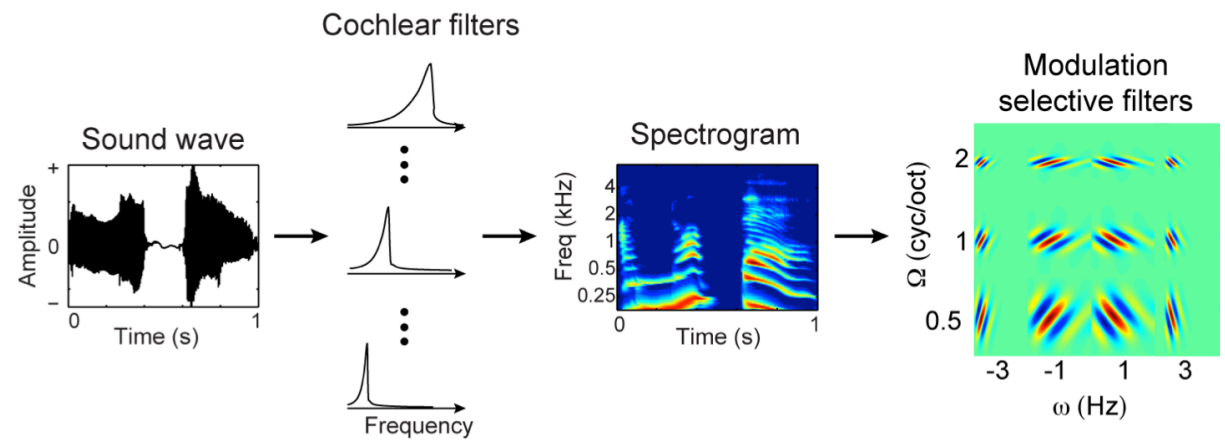

Figure 1.4. Schematic of the computational model of auditory processing. The sound wave enters the peripheral stage, where it is analyzed by a bank of band pass cochlear-like filters (a schematic of the filters' transfer function is depicted in the figure). Further transformations mimicking hair cells and midbrain mechanisms (not shown here - see Chi et al., 2005) generate the auditory spectrogram. Finally, the cortical stage computes the modulation content of the spectrogram using an array of modulation selective filters tuned to different values of spectral and temporal modulations frequencies. Impulse responses are shown for a selection of cortical filters. 


\section{Outline of the thesis}

Chapter 2 employs an encoding approach to investigate the cortical processing of spectral and temporal modulations and its relation to the frequencyspecific responses observed throughout the auditory cortex. Competing implementations of a computational model that incorporates sensitivity to frequency, spectral and temporal modulations are compared. This chapter additionally investigates the spatial distribution of voxels' spectral and temporal modulation tuning properties by computing maps of modulation frequency preference. The spatial layout of spectral and temporal response properties throughout the auditory cortex is further investigated in Chapter 3. The study described in this chapter employs a decoding procedure to characterize the spectral and temporal computational profile of distinct, anatomically defined regions of interest. Compared to the maps of modulation preference described in Chapter 2, the model-based reconstruction approach implemented in Chapter 3 provides a more direct measure of which acoustic features are faithfully encoded within a given cortical region (see Mesgarani et al., 2009, and Chapter 5 for an indepth discussion about this topic). A second aim of Chapter 3 is to assess the relevance of the observed spectral and temporal response properties for the discriminability of natural sounds. Chapter 4 builds on findings and methodological techniques of Chapters 2 and 3 to achieve brain-based re-synthesis of natural sounds based on a time-resolved implementation of a model of spectro-temporal processing. Finally, Chapter 5 summarizes the findings of this thesis, discusses the methods employed, and outlines future research directions.

\section{References}

Ahveninen J, Jääskeläinen IP, Raij $T$, Bonmassar $G$, Devore $S$, Hämäläinen $M$, Levänen S, Lin F-H, Sams M, Shinn-Cunningham BG, Witzel T, Belliveau JW (2006) Task-modulated "what" and "where" pathways in human auditory cortex. Proc Natl Acad Sci U S A 103:14608-14613

Altmann CF, Bledowski C, Wibral M, Kaiser J (2007) Processing of location and pattern changes of natural sounds in the human auditory cortex. Neuroimage 35:1192-1200 
Bacon SP (1989) Modulation masking: Effects of modulation frequency, depth, and phase. J Acoust Soc Am 85:2575

Bacon SP, Viemeister NF (1985) Temporal modulation transfer functions in normalhearing and hearing-impaired listeners. Audiology 24:117-134

Barton B, Venezia J (2012) Orthogonal acoustic dimensions define auditory field maps in human cortex. Proc Natl Acad Sci U S A 109:20738-20743

Belin P, Zatorre RJ, Lafaille P, Ahad P, Pike B (2000) Voice-selective areas in human auditory cortex. Nature 403:309-312

Bitterman Y, Mukamel R, Malach R, Fried I, Nelken I (2008) Ultra-fine frequency tuning revealed in single neurons of human auditory cortex. Nature 451:197201

Bonte M, Hausfeld L, Scharke W, Valente G, Formisano E (2014) Task-dependent decoding of speaker and vowel identity from auditory cortical response patterns. J Neurosci 34:4548-4557

Chi T, Gao Y, Guyton MC, Ru P, Shamma S (1999) Spectro-temporal modulation transfer functions and speech intelligibility. J Acoust Soc Am 106:2719-2732

Chi T, Ru P, Shamma S (2005) Multiresolution spectrotemporal analysis of complex sounds. J Acoust Soc Am 118:887-906

deCharms RC (1998) Optimizing Sound Features for Cortical Neurons. Science (80) 280:1439-1444

Depireux DA, Simon JZ, Klein DJ, Shamma SA, Ding N (2001) Spectro-Temporal Response Field Characterization With Dynamic Ripples in Ferret Primary Auditory Cortex. J Neurophysiol 85:1220-1234.

Drullman R, Festen J, Plomp R (1994) Effect of temporal envelope smearing on speech reception. J Acoust Soc Am 95:1053-1064

Eddins DA, Bero EM (2007) Spectral modulation detection as a function of modulation frequency, carrier bandwidth, and carrier frequency region. J Acoust Soc Am 121:363

Elliott TM, Theunissen FE (2009) The modulation transfer function for speech intelligibility. PLoS Comput Biol 5:e1000302 


\section{Chapter 1}

Ewert SD, Dau T (2000) Characterizing frequency selectivity for envelope fluctuations. J Acoust Soc Am 108:1181-1196

Formisano E, De Martino F, Bonte M, Goebel R (2008) "Who" Is Saying "What"? Brain-Based Decoding of Human Voice and Speech. Sci 322 :970-973

Giraud AL, Lorenzi C, Ashburner J, Wable J, Johnsrude I, Frackowiak R, Kleinschmidt A (2000) Representation of the temporal envelope of sounds in the human brain. J Neurophysiol 84:1588-1598

Goldstein B (2014) Sensation and Perception. Cengage Learning.

Green D (1986) "Frequency" and the Detection of Spectral Shape Change. In: Auditory Frequency Selectivity (Moore BJ, Patterson R, eds), pp 351-359 Nato ASI Series. Springer US.

Herdener M, Esposito F, Scheffler K, Schneider P, Logothetis NK, Uludag K, Kayser C (2013) Spatial representations of temporal and spectral sound cues in human auditory cortex. Cortex 49:2822-2833

Houtgast T (1989) Frequency selectivity in amplitude-modulation detection. J Acoust Soc Am 85:1676

Joris PX, Schreiner CE, Rees A (2004) Neural processing of amplitude-modulated sounds. Physiol Rev 84:541-577

Kowalski N, Depireux DA, Shamma SA (1996) Analysis of dynamic spectra in ferret primary auditory cortex. I. Characteristics of single-unit responses to moving ripple spectra. J Neurophysiol 76:3503-3523.

Langers DRM, Backes WH, Dijk P Van (2003) Spectrotemporal features of the auditory cortex: the activation in response to dynamic ripples. Neuroimage 20:265-275

Laudanski J, Edeline J-M, Huetz C (2012) Differences between Spectro-Temporal Receptive Fields Derived from Artificial and Natural Stimuli in the Auditory Cortex. PLoS One 7:e50539

Lewis JW, Brefczynski JA, Phinney RE, Janik JJ, DeYoe EA (2005) Distinct cortical pathways for processing tool versus animal sounds. J Neurosci 25:5148-5158 
Liégeois-Chauvel C, Lorenzi C, Trébuchon A, Régis J, Chauvel P (2004) Temporal envelope processing in the human left and right auditory cortices. Cereb Cortex 14:731-740

Liu C, Eddins DA (2008) Effects of spectral modulation filtering on vowel identification. J Acoust Soc Am 124:1704-1715

Logothetis NK, Pauls J, Augath M, Trinath T, Oeltermann A (2001) Neurophysiological investigation of the basis of the fMRI signal. Nature 412:150-157

Mesgarani N, David S V, Fritz JB, Shamma S a (2009) Influence of context and behavior on stimulus reconstruction from neural activity in primary auditory cortex. J Neurophysiol 102:3329-3339

Miyawaki Y, Uchida H, Yamashita O, Sato M, Morito Y, Tanabe HC, Sadato N, Kamitani $Y$ (2008) Visual image reconstruction from human brain activity using a combination of multiscale local image decoders. Neuron 60:915-929

Naselaris T, Kay KN, Nishimoto S, Gallant JL (2010) Encoding and decoding in fMRI. Neuroimage:1-11

Naselaris T, Prenger RJ, Kay KN, Oliver M, Gallant JL (2009) Bayesian reconstruction of natural images from human brain activity. Neuron 63:902915

Nishimoto S, Vu AT, Naselaris T, Benjamini Y, Yu B, Gallant JL (2011) Reconstructing Visual Experiences from Brain Activity Evoked by Natural Movies. Curr Biol:1641-1646

O'Connor KN, Johnson JS, Niwa M, Noriega NC, Marshall EA, Sutter ML (2011) Amplitude modulation detection as a function of modulation frequency and stimulus duration: comparisons between macaques and humans. Hear Res 277:37-43

O'Doherty JP, Hampton A, Kim H (2007) Model-based fMRI and its application to reward learning and decision making. Ann N Y Acad Sci 1104:35-53

Ogawa S, Tank DW, Menon R, Ellermann JM, Kim SG, Merkle H, Ugurbil K (1992) Intrinsic signal changes accompanying sensory stimulation: functional brain mapping with magnetic resonance imaging. Proc Natl Acad Sci U S A 89:5951-5955 


\section{Chapter 1}

Overath T, Zhang Y, Sanes DH, Poeppel D (2012) Sensitivity to temporal modulation rate and spectral bandwidth in the human auditory system: fMRI evidence. J Neurophysiol:1-2

Rosen S (1992) Temporal information in speech: acoustic, auditory and linguistic aspects. Philos Trans R Soc Lond B Biol Sci 336:367-373

Sabin AT, Eddins DA, Wright BA (2012) Perceptual Learning Evidence for Tuning to Spectrotemporal Modulation in the Human Auditory System. J Neurosci 32:6542-6549

Samson F, Zeffiro TA, Toussaint A, Belin P (2011) Stimulus complexity and categorical effects in human auditory cortex: an activation likelihood estimation meta-analysis. Front Psychol 1

Saoji AA, Eddins DA (2007) Spectral modulation masking patterns reveal tuning to spectral envelope frequency. J Acoust Soc Am 122:1004-1013

Schoenmakers S, Barth M, Heskes T, van Gerven M (2013) Linear reconstruction of perceived images from human brain activity. Neuroimage 83:951-961

Schönwiesner M, Rübsamen R, von Cramon DY (2005) Hemispheric asymmetry for spectral and temporal processing in the human antero-lateral auditory belt cortex. Eur J Neurosci 22:1521-1528

Schönwiesner M, Zatorre RJ (2009) Spectro-temporal modulation transfer function of single voxels in the human auditory cortex measured with high-resolution fMRI. Proc Natl Acad Sci U S A 106:14611-14616

Schreiner CE, Calhoun BM (1994) Spectral envelope coding in cat primary auditory cortex: Properties of ripple transfer functions. Aud Neurosci:39-61

Schreiner CE, Froemke RC, Atencio CA (2011) Spectral Processing in Auditory Cortex. In: The Auditory Cortex, 1st ed. (Winer JA, Schreiner CE, eds), pp 275-308. Springer.

Schreiner CE, Winer JA (2007) Auditory cortex mapmaking: principles, projections, and plasticity. Neuron 56:356-365

Shamma S, Versnel H, Kowalski N (1995) Ripple Analysis in Ferret Primary Auditory Cortex. I. Response Characteristics of Single Units to Sinusoidally Rippled Spectra. Aud Neurosci 1:233-254 
Shannon R V, Zeng F-G, Kamath V, Wygonski J, Ekelid M (1995) Speech Recognition with Primarily Temporal Cues. Sci $270: 303-304$

Singh NC, Theunissen FE (2003) Modulation spectra of natural sounds and ethological theories of auditory processing. J Acoust Soc Am 114:3394-3411

Staeren N, Renvall H, De Martino F, Goebel R, Formisano E (2009) Sound categories are represented as distributed patterns in the human auditory cortex. Curr Biol 19:498-502

Tansley BW (1983) Time course of adaptation and recovery of channels selectively sensitive to frequency and amplitude modulation. J Acoust Soc Am 74:765

Theunissen E, Sen K, Doupe AJ (2000) Spectral-Temporal Receptive Fields of Nonlinear Auditory Neurons. J Neurosci 20:2315-2331.

Theunissen FE, Elie JE (2014) Neural processing of natural sounds. Nat Rev Neurosci 15:355-366

Thirion B, Duchesnay E, Hubbard E, Dubois J, Poline J-B, Lebihan D, Dehaene S (2006) Inverse retinotopy: inferring the visual content of images from brain activation patterns. Neuroimage 33:1104-1116

Versnel H, Kowalski N, Shamma SA (1995) Ripple Analysis in Ferret Primary Auditory Cortex . III . Topographic Distribution of Ripple Response Parameters. Aud Neurosci 1:271-285.

Versnel H, Shamma S a (1998) Spectral-ripple representation of steady-state vowels in primary auditory cortex. J Acoust Soc Am 103:2502-2514

Viemeister NF (1979) Temporal modulation transfer functions based upon modulation thresholds. J Acoust Soc Am 66:1364-1380

Wang X, Merzenich MM, Beitel R, Schreiner CE (1995) Representation of a speciesspecific vocalization in the primary auditory cortex of the common marmoset: temporal and spectral characteristics. J Neurophysiol 74:2685-2706

Warrier C, Wong P, Penhune V, Zatorre R, Parrish T, Abrams D, Kraus N (2009) Relating structure to function: Heschl's gyrus and acoustic processing. J Neurosci 29:61-69 
Chapter 1

Wu MC-K, David S V, Gallant JL (2006) Complete functional characterization of sensory neurons by system identification. Annu Rev Neurosci 29:477-505

Zatorre RJ, Belin P (2001) Spectral and Temporal Processing in Human Auditory Cortex. Cereb Cortex 11 :946-953 


\section{Chapter 2}

\section{Encoding of Natural Sounds at Multiple Spectral and Temporal Resolutions in the Human Auditory \\ Cortex}

Corresponding publication:

Santoro R, Moerel M, De Martino F, Goebel R, Ugurbil K, Yacoub E, Formisano E (2014) Encoding of Natural Sounds at Multiple Spectral and Temporal Resolutions in the Human Auditory Cortex. PLoS Comput Biol 10(1): e1003412. doi:10.1371/journal.pcbi.1003412 


\section{Abstract}

Functional neuroimaging research provides detailed observations of the response patterns that natural sounds (e.g. human voices and speech, animal cries, environmental sounds) evoke in the human brain. The computational and representational mechanisms underlying these observations, however, remain largely unknown. Here we combine high spatial resolution (3 and 7 Tesla) functional magnetic resonance imaging ( $\mathrm{fMRI}$ ) with computational modeling to reveal how natural sounds are represented in the human brain. We compare competing models of sound representations and select the model that most accurately predicts fMRI response patterns to natural sounds. Our results show that the cortical encoding of natural sounds entails the formation of multiple representations of sound spectrograms with different degrees of spectral and temporal resolution. The cortex derives these multi-resolution representations through frequency-specific neural processing channels and through the combined analysis of the spectral and temporal modulations in the spectrogram. Furthermore, our findings suggest that a spectral-temporal resolution trade-off may govern the modulation tuning of neuronal populations throughout the auditory cortex. Specifically, our fMRI results suggest that neuronal populations in posterior/dorsal auditory regions preferably encode coarse spectral information with high temporal precision. Vice-versa, neuronal populations in anterior/ventral auditory regions preferably encode fine-grained spectral information with low temporal precision. We propose that such a multi-resolution analysis may be crucially relevant for flexible and behaviorally-relevant sound processing and may constitute one of the computational underpinnings of functional specialization in auditory cortex. 


\section{Introduction}

Understanding how natural sounds and scenes are processed in the human auditory cortex remains a major challenge in auditory neuroscience. Current models of auditory cortical processing describe the sound-evoked neural response patterns at the level of preferential regional activations for certain behavioral tasks (e.g. localization vs recognition; Alain et al., 2001; Ahveninen et al., 2006), sound categories (e.g. voices, speech; Belin et al., 2000) and (complex) acoustic features (Lewis et al., 2009; Leaver and Rauschecker, 2010). However, the computational and representational mechanisms underlying these responses remain largely unknown. The overall aim of the present study is to derive a computational model of how natural sounds are encoded in the human brain by combining highresolution $\mathrm{fMRI}$ ( 3 and 7 Tesla) with computational modelling.

Most natural sounds are characterized by modulations of acoustic energy in both the spectral and temporal dimensions (Figure 2.1A). These modulations occur at multiple scales (Singh and Theunissen, 2003) and are crucial for behaviorally relevant auditory processing such as speech intelligibility (Drullman et al., 1994; Shannon et al., 1995; Chi et al., 1999; Elliott and Theunissen, 2009). Psychophysical investigations indicate that humans are able to detect and discriminate modulations that occur in one dimension alone (temporal: Viemeister, 1979; spectral: Green, 1986) as well as combined spectro-temporal modulations (Chi et al., 1999). Similarly, neurophysiological studies in animals and humans have revealed neuronal tuning for temporal modulations (Giraud et al., 2000; Joris et al., 2004; Barton and Venezia, 2012) and spectral modulations (Shamma et al., 1995) alone, and the combination of the two (Kowalski et al., 1996; Depireux et al., 2001; Langers et al., 2003; Woolley et al., 2005; Schönwiesner and Zatorre, 2009). This evidence suggests that spectral and temporal modulations are critical stimulus dimensions for the processing of sounds in the auditory cortex. Just as the cochlea generates multiple "views" of the sound pressure wave at different frequencies, an explicit encoding of spectral and temporal modulations would allow the cortex generating multiple "views" of the sound spectrogram with different degrees of spectral and temporal resolution (Chi et al., 2005) (Figure 2.1B). Multiple simultaneous representations of the same incoming sounds may be crucially relevant for enabling flexible behavior, as different goal-oriented sound processing (e.g. sound localization or identification) may benefit from different types of representations. Furthermore, the representations of sounds at multiple resolutions may provide the computational basis for binding acoustic elements in sound mixtures and solve complex auditory scenes (Elhilali and Shamma, 2008). 
A
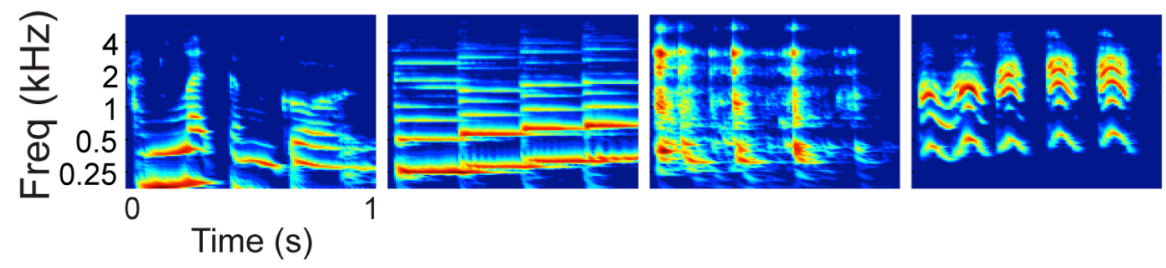

B

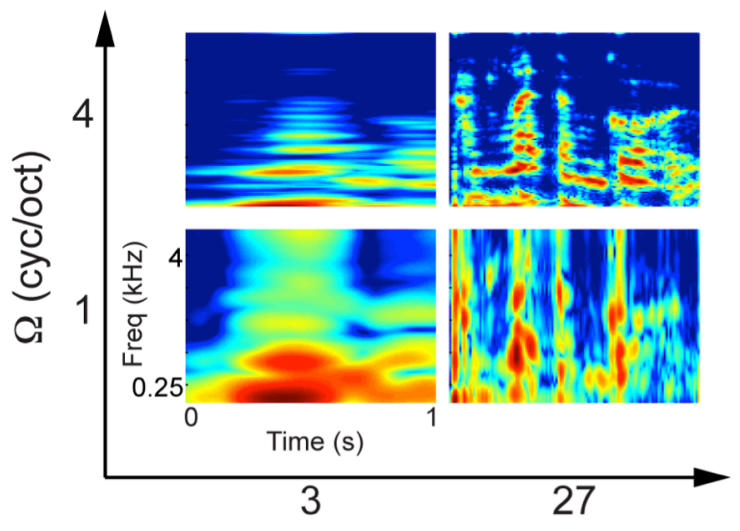

$\omega(\mathrm{Hz})$

Figure 2.1. Sound examples and multi-resolution decomposition. (A) Spectrogram of four exemplary natural sounds used in this study as extracted by the computational model mimicking early auditory processing. Natural sounds exhibit modulations of acoustic energy along both frequency and time. (B) Multi-resolution representation of the leftmost spectrogram of panel A. Different "views" are obtained as output of modulation channels tuned to specific spectral modulation $(\Omega)$ and temporal modulation $(\omega)$ frequencies. Each channel represents the spectrogram with a different combination of spectral and temporal detail.

Despite extensive investigations in a variety of experimental settings, the specific computational mechanisms used by the human auditory cortex to represent energy modulations in the spectrogram of natural sounds are still a matter of speculation. Here, we use an fMRI "encoding" approach (Kay et al., $2008 \mathrm{~b})$ to compare competing computational models of sound representations and select the best model as the one that can predict most accurately fMRI response patterns to natural sounds. We focus on three well-defined aspects of the representation of spectral and temporal modulations: (1) dependency, (2) frequency specificity, and (3) spatial organization. 
Dependency refers to the relation between spectral and temporal processing. The spectrogram of natural sounds is characterized by concurrent spectral and temporal modulations and these sound qualities might be represented jointly or independently of each other. An independent representation implies separate processing mechanisms for spectral and temporal modulations, such that the response to one dimension is invariant to a change in the other dimension. By contrast, a joint representation relies on combined selectivity for the conjunction of spectral and temporal modulations. The joint representation can be modeled as an array of spectro-temporal filters that are selective for combinations of spectral and temporal modulations (Figure S2.1A), whereas the independent representation can be seen as a bank of filters that are selective for either temporal or spectral modulations (Figure S2.1B). In other words, the two models differ with respect to the dimensions employed by the auditory cortex to encode natural sounds (combined spectro-temporal modulations, and spectral and temporal modulations alone, respectively). Testing for the interdependency of spectral and temporal modulation processing has relevant implications, as the superiority of such a model would indicate that results obtained using sounds that only vary along one dimension (e.g. amplitude modulated tones or stationary ripples) cannot be generalized to mechanisms of representation and processing of natural sounds.

The analysis of the spectro-temporal modulation content of the sound spectrogram can be global (2D Fourier transform) or localized (e.g wavelet transform). A global representation indicates integration along the frequency axis, while in a local analysis spectral and temporal modulations are encoded in a frequency-specific fashion. Frequency specific responses are ubiquitous in the auditory cortex; yet it is not clear how this dimension is exploited for the representation of natural sounds. Understanding the nature of the modulation analysis performed by the human auditory cortex can provide insights about the functional role of this representational mechanism.

Finally, the third aspect that we consider is the existence and layout of a large-scale spatial organization of spectro-temporal modulation tuning. Topographic maps of stimulus dimensions are a well-established organizational principle of the auditory cortex (Schreiner and Winer, 2007). In humans, the primary (Formisano et al., 2003) as well as the non-primary (Moerel et al., 2012) auditory cortex contain multiple topographic representations of sound frequency (tonotopic maps). Beyond tonotopy, however, the spatial organization of other sound features remains elusive (Schreiner and Winer, 2007). Our methodological approach provides the possibility to obtain maps of multiple sound features and feature- 
combinations from the same set of $\mathrm{fMRI}$ responses and within the ecologically and behaviorally-relevant context of natural sounds processing. Here, we exploit this possibility to study the regional specificity and the spatial organization of spectrotemporal modulation tuning. Such knowledge can reveal the representational and computational basis underlying the functional specialization of auditory cortical subdivisions.

Our results show that the human brain forms multiple representations of incoming natural sounds at distinct spectral and temporal resolutions. The encoding of spectral and temporal modulations is joint and frequency-specific and is governed by a trade-off between spectral and temporal resolution. Regional variations of voxels modulation preference put forward the hypothesis that the functional specialization of auditory cortical fields can be partially accounted for by their modulation tuning.

\section{Results}

We modeled the data from two fMRI experiments in humans (3T (Moerel et al., 2012) and 7T (De Martino et al., 2013; Moerel et al., 2013)). In both experiments, fMRI responses were recorded from the auditory cortex while subjects ( $n=5$, different for the two experiments) listened to a large set of natural sounds, including speech samples, music pieces, animal cries, scenes from nature, and tool sounds (see Materials and Methods and Supplementary Methods).

\section{Prediction accuracy of the joint frequency-specific MTF-based model}

We applied an "encoding" approach (see (Kay et al., 2008b) and Figure S2.2) and compared several computational models of auditory processing. A first model we tested describes auditory cortical neurons as a bank of frequency-localized filters with joint selectivity for spectral and temporal modulations (see (Chi et al., 2005) and Materials and Methods). Considering that one voxel reflects the mass activity of a great number of neurons, we modelled each voxel's receptive field as a combination of modulation selective filters, each tuned to a different spectral modulation, temporal modulation and frequency (Figure 2.2, panel A). Using a subset of $\mathrm{fMRI}$ data (training), we estimated a modulation transfer function (MTF, Figure 2.2, panel A1) for each voxel (see Figure 2.3 for two MTF examples). We then assessed the ability of this MTF-based model to accurately predict the fMRI responses in new, independent data sets (testing). In the $3 T$ experiment, training and testing data involved a single set of natural sounds, whereas two completely 
distinct sound sets were used for the $7 \mathrm{~T}$ training and testing datasets. We quantified model's prediction accuracy by performing a sound identification analysis (Kay et al., 2008b). Namely, we used the fMRI activity patterns predicted by the estimated models to identify which sound had been heard among all sounds in the test set. Each testing sound was assigned with a score ranging between 0 and 1 and indicating the rank of the correlation between sound's predicted and measured activity patterns ( 0 indicates that the predicted activity pattern for a given stimulus was least similar to the measured one among all test stimuli; 1 indicates correct identification). The overall model's accuracy was obtained as the average score across all test sounds (see Materials and Methods).

For both the $3 \mathrm{~T}$ and $7 \mathrm{~T}$ datasets, the accuracy of the joint frequency-specific MTF-based model was significantly higher than chance (0.5) both at group level (3T: mean $[\mathrm{SE}]=0.66$ [0.02], $\mathrm{p}=0.003 ; 7 \mathrm{~T}$ : mean [SE] $=0.78$ [0.03], $\mathrm{p}=0.002$; twotailed paired t-test; Figure 2.4) and for each individual subject ( $p=0.01$ for subject S4, $p=0.005$ for all other subjects, permutation test; Figure 2.5). Remarkably, for the 7T dataset the joint frequency-specific MTF-based model was able to generalize to stimuli not used for parameter estimation.

Comparison between joint frequency-specific MTF-based model and tonotopy model

FMRI activity from voxels in primary and non-primary auditory regions reflects the tonotopic organization of neural responses. Therefore, as a control analysis we compared the prediction accuracy of the MTF-based model against the prediction accuracy of a tonotopy model, which incorporates the hypothesis that voxels simply reflect information about the frequency content of the stimuli (see Materials and Methods and Figure 2.2, panel C). The tonotopy model performed above chance both at group level (3T: mean $[\mathrm{SE}]=0.62[0.02], \mathrm{p}=0.002 ; 7 \mathrm{~T}$ : mean $[\mathrm{SE}]=$ 0.69 [0.03], $p=0.004$; two-tailed paired t-test; Figure 2.4) and for each individual subject ( $p=0.015$ for subject $S 4, p=0.005$ for all other subjects, permutation test; Figure 2.5). However, the tonotopy model performed significantly worse than the joint frequency-specific MTF-based model (3T: $p=0.009 ; 7 \mathrm{~T}: \mathrm{p}=0.007$; two-tailed paired t-test). The significant improvement of the MTF-based over the tonotopy model indicates that a model accounting for the joint, frequency-specific modulation content of the spectrogram is a better representation of $\mathrm{fMRI}$ responses to natural sounds. 


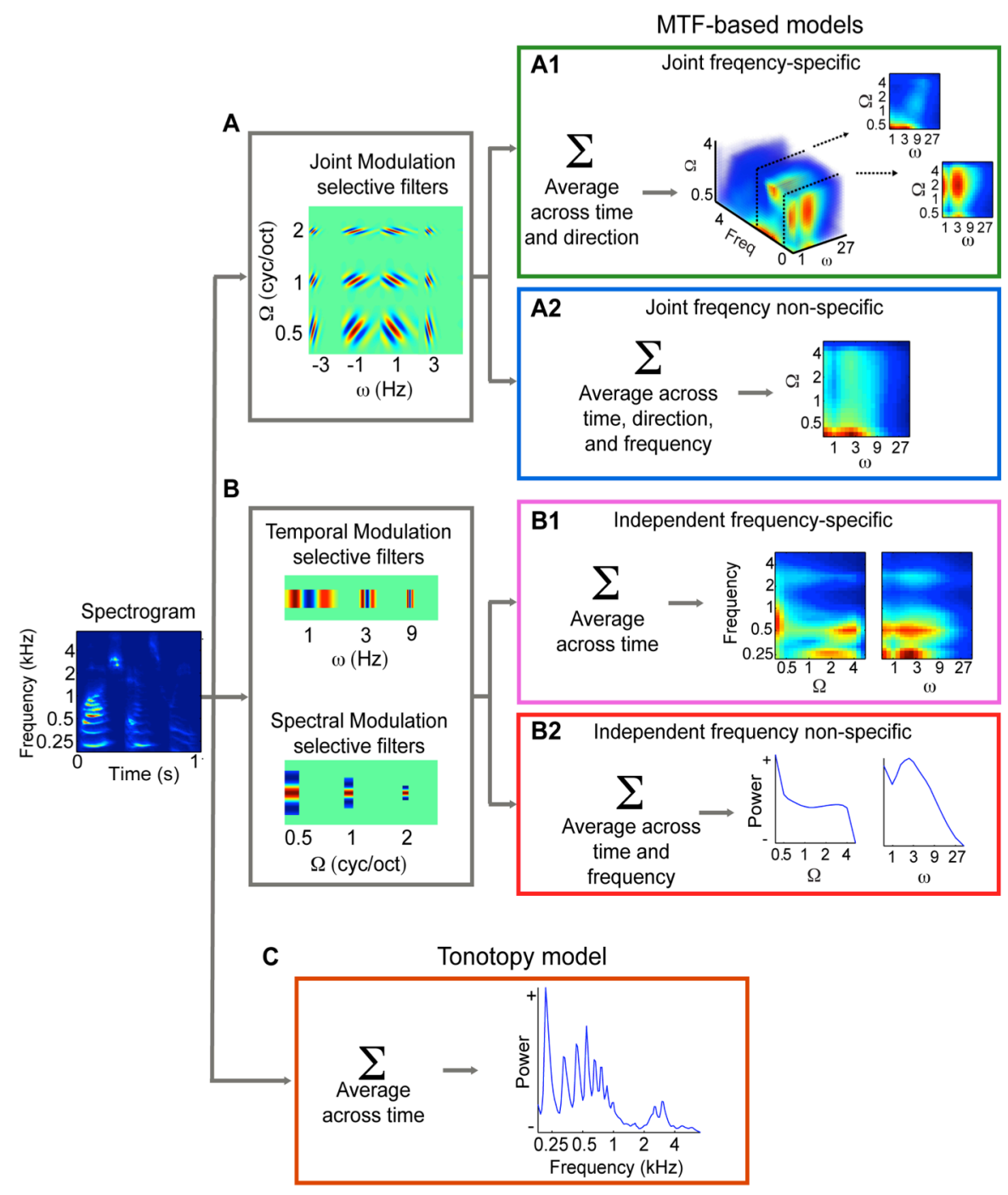

Figure 2.2. Overview of candidate encoding models. (A) Joint modulation selective filters. (A1) Joint frequency specific: the spectrogram is filtered with a bank of modulation selective filters at different spectral modulations $(\Omega)$, temporal modulations $(\omega)$, and direction (upwards/downwards). The output of the filter bank is averaged across time and direction to yield a reduced representation of modulation energy as a function of $\Omega, \omega$, and frequency. The joint frequency-specific MTF-based model predicts that $\mathrm{fMRI}$ responses vary linearly with this representation, i.e. sounds that differ with respect to any of the three dimensions will elicit different responses. (A2) Joint frequency non-specific: the 3D modulation representation is averaged across frequency to yield a global measure of modulation energy. By concatenating modulation and frequency content (not shown here, see tonotopy model), the ... 
... joint frequency non-specific model predicts separate processing for global, joint modulations and frequency. (B) Independent modulation selective filters. (B1) Independent frequency-specific: the spectrogram is filtered with purely spectral and purely temporal modulation selective filters and the output is averaged over time. This yields separate representations of spectral and temporal modulation energy as a function of frequency. The independent frequency-specific model predicts that the response of a voxel dedicated to spectral (temporal) processing will not be affected by a change in temporal (spectral) modulation content. (B2) Independent frequency non-specific: the two separate representations of spectral and temporal modulation energy are averaged across frequency to yield the global spectral and temporal modulation content. This representation is concatenated with the frequency content (not shown here, see tonotopy model) to simulate separate processing for frequency, spectral and temporal modulations. (C) Tonotopy model: the spectrogram is averaged over time and voxels are modeled as frequency selective units, whose response varies linearly with the frequency content of the input stimuli.

Comparison between frequency-specific and non-specific joint MTF-based models

To assess the relevance of frequency-localization in the encoding of joint spectro-temporal modulations, we trained a model that represents frequency and joint modulation content independently of each other (see Materials and Methods and Figure 2.2, panel A2). The joint frequency non-specific MTF-based model performed above chance both at group level (3T: mean [SE] $=0.63$ [0.02], $p=$ 0.004; 7T: mean [SE] $=0.71$ [0.02], $p=0.0003$; two-tailed paired t-test; Figure 2.4) and for each individual subject $(p=0.02$ for subject $S 4, p=0.01$ for subject $S 6, p=$ 0.005 for all other subjects, permutation test). However, the frequency non-specific model performed significantly worse than the frequency-specific MTF-based model (3T: $p=0.002 ; 7 \mathrm{~T}: \mathrm{p}=0.021$; two-tailed paired t-test).

Comparison between joint and independent frequency-specific MTF-based models

In order to quantify the contribution of joint selectivity to identification performance, we trained an independent frequency-specific MTF-based encoding model. We modelled each voxel's receptive field as a combination of purely temporal and purely spectral modulation selective filters, operating in a frequencyspecific fashion (see Materials and Methods and Figure 2.2, panels B and B1). The independent model performed above chance both at group level (3T: mean [SE] = 0.63 [0.01], $p=0.001 ; 7 \mathrm{~T}:$ mean $[\mathrm{SE}]=0.72$ [0.02], $p=0.0007$; two-tailed paired t- 
test; Figure 2.4) and for each individual subject ( $p=0.015$ for subject $S 4, p=0.01$ for subject S7, $p=0.005$ for all other subjects, permutation test). However, the independent model performed significantly worse than the joint MTF-based model (3T: $p=0.012 ; 7 T: p=0.011$; two-tailed paired t-test).
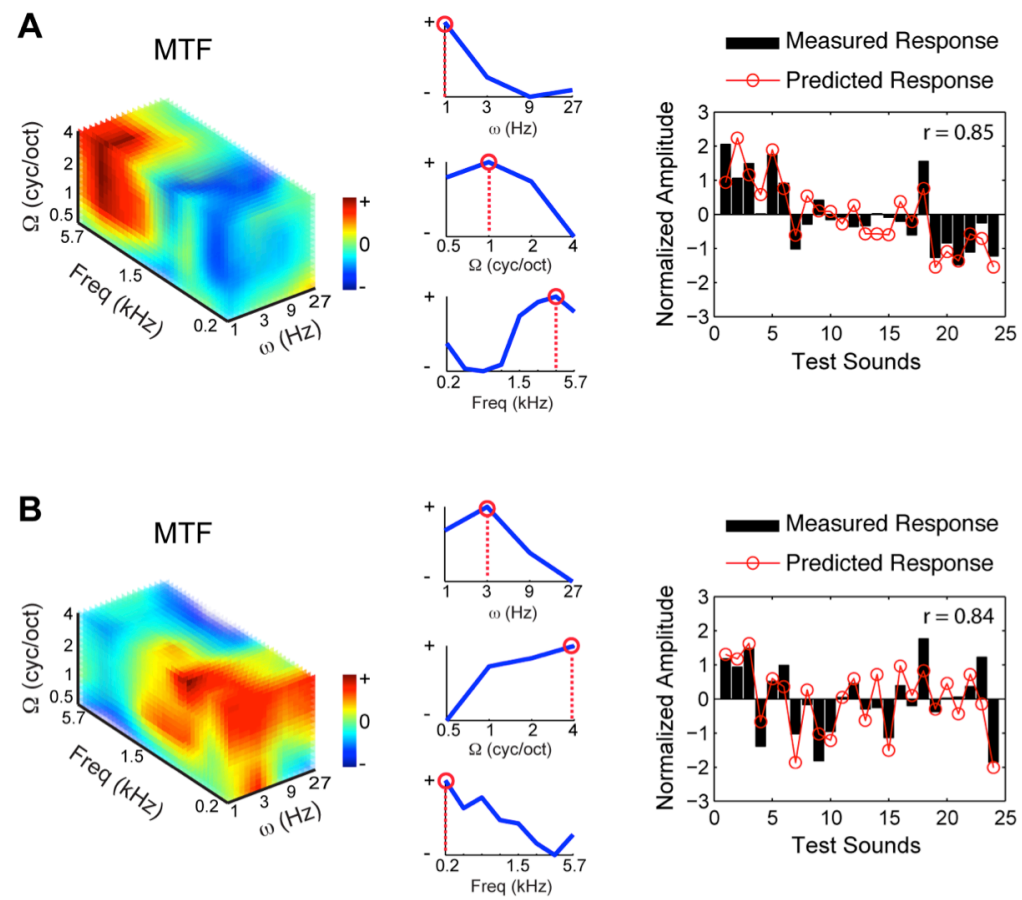

Figure 2.3. Sample MTFs, data and model prediction. (A-B) Left: MTFs as estimated by the joint frequency-specific MTF-based model for two exemplary voxels (subject S10). The color code indicates the voxel's sensitivity to a given combination of frequency, spectral and temporal modulation. MTFs have been interpolated for display purposes. Middle: Marginal response profiles for temporal modulation (top), spectral modulation (middle) and frequency (bottom). Red circles and dashed lines indicate voxels' characteristic spectral modulation, temporal modulation and frequency, computed as the point of maximum of the marginal profiles (see Materials and Methods). Right: Measured and predicted response to the 24 stimuli in the test set. Responses are shown in z-score units. $r$ indicates Pearson's correlation coefficient.

Comparison between joint frequency-specific and independent frequency nonspecific MTF-based models

As an additional control, we tested a model that simulates independent selectivity for spectral modulations, temporal modulations and frequency (see 
Materials and Methods and Figure 2.2, panel B2). The independent frequency nonspecific model performed above chance both at group level (3T: mean [SE] $=0.63$ [0.02], $\mathrm{p}=0.002 ; 7 \mathrm{~T}$ : mean [SE] $=0.71$ [0.02], $\mathrm{p}=0.0008$; two-tailed paired t-test; Figure 2.4) and for each individual subject ( $p=0.01$ for subject $S 1, S 4$ and S9, $p=$ 0.005 for all other subjects, permutation test). However, the independent frequency non-specific model performed significantly worse than the joint frequency-specific MTF-based model (3T: $p=0.011$; 7T: $p=0.016$; two-tailed paired t-test).

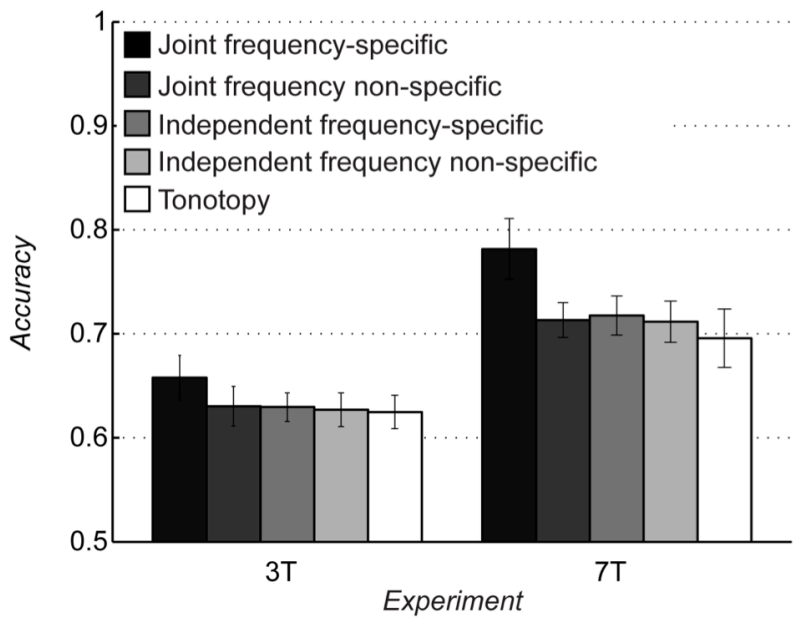

Figure 2.4. Comparison between models. Bars indicate the prediction accuracy (mean $\pm \mathrm{SEM}, \mathrm{N}=5$ ) for the five models in both the $3 \mathrm{~T}$ and $7 \mathrm{~T}$ experiments. The joint frequency-specific MTF-based model showed significantly better prediction accuracy than all other models (see main text). Accuracies are normalized between 0 and 1. Chance level is 0.5 .

\section{Spatial distribution of voxels' tuning properties}

To investigate the cortical topography of voxels tuning properties, we computed maps of voxels characteristic spectral modulation (CSM), temporal modulation (CTM) and frequency (CF). For each feature, the estimated MTF was marginalized across irrelevant dimensions (i.e. spectral and temporal modulations for (F) and the point of maximum of the marginal sum was assigned as the voxel's preferred feature value (see example in Figure 2.3). We obtained maps of CSM, CTM and CF by color-coding the voxels' preferred values and projecting them onto an inflated representation of the subject's cortex (see Materials and Methods). Maps of CF confirmed the presence of multiple tonotopic gradients in primary 
A

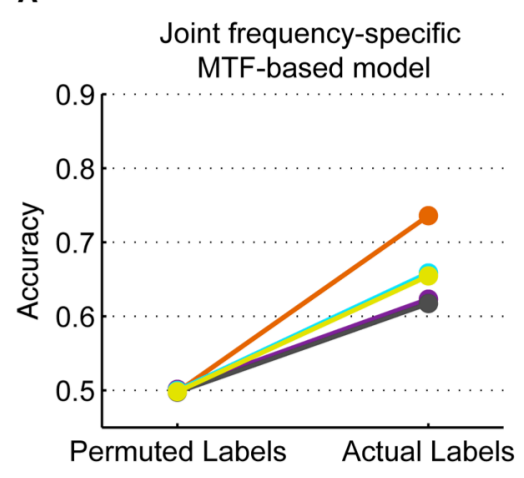

Tonotopy model

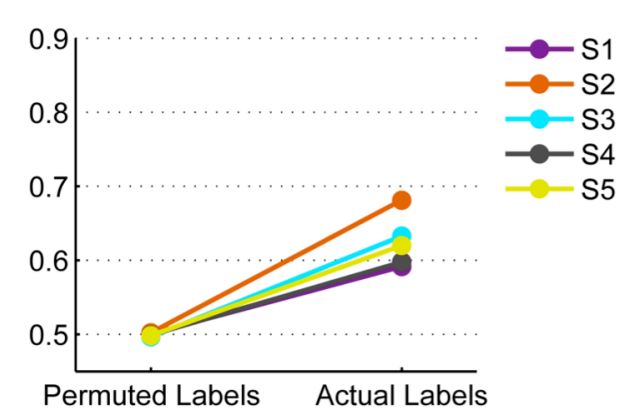

B

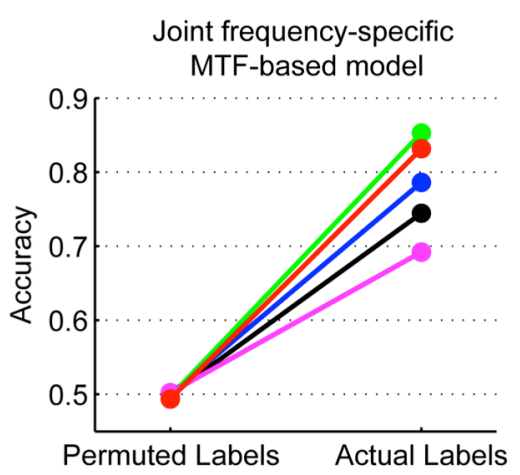

Tonotopy model

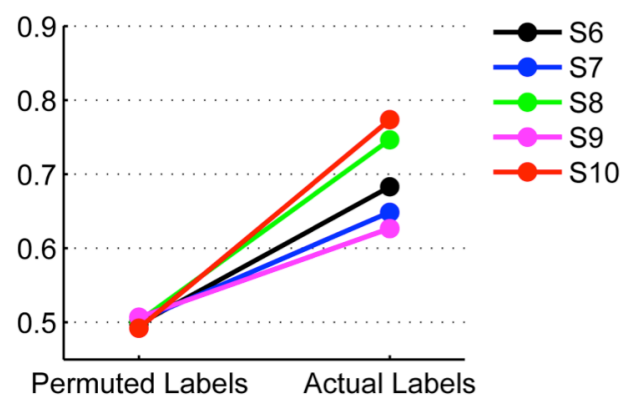

Figure 2.5. Prediction accuracies for individual participants. Accuracies of the joint frequency-specific MTF-based (left) and tonotopy (right) models are reported for the 3T (A) and 7T (B) datasets. Each panel shows the accuracy obtained with correct labels and the accuracy derived by permuting the sound labels before training the model.

auditory regions (Heschl's gyrus - HG) and surrounding superior temporal cortex (Moerel et al., 2012) (Figure S2.3 and S2.4). The spatial distribution of voxels CSM and CTM appeared to be more complex and variable across subjects (Figure 2.6 for the group and Figure S2.5 and S2.6 for all individual subjects). However, the group data and the majority of the individual subjects suggested distinct regional sensitivities to modulation frequencies (see schematic summary in Figure 2.7). In both hemispheres, clusters with a preference for fine spectral modulations (high CSM, purple colors) were primarily and consistently localized along the HG and anterior superior temporal gyrus (STG) (see circles on group maps - Figure 2.6), while clusters with a preference for coarse spectral modulations (low CSM, orange 
color) were mostly located posterior-laterally to $\mathrm{HG}$, on the planum temporal (PT) and on STG (see squares on group maps - Figure 2.6). Bilaterally, a preference for slow temporal modulations (low CTM, orange color) was found along HG and STG, whereas clusters with a preference for fast temporal modulations (high CTM, purple) were observed on the PT, posteriorly to $\mathrm{HG}$ and in a region medially adjacent to HG. Supporting the spatial dissociation between spectral and temporal modulation at map level, we found a significant negative correlation between voxels characteristic spectral and temporal modulation (3T: mean $[\mathrm{SE}]=-0.19$ [0.01], $p=0.02 ; 7 \mathrm{~T}$ : mean $[\mathrm{SE}]=-0.11$ [0.01], $\mathrm{p}=0.01$; group level random effects two-tailed t test, see Materials and Methods).

A
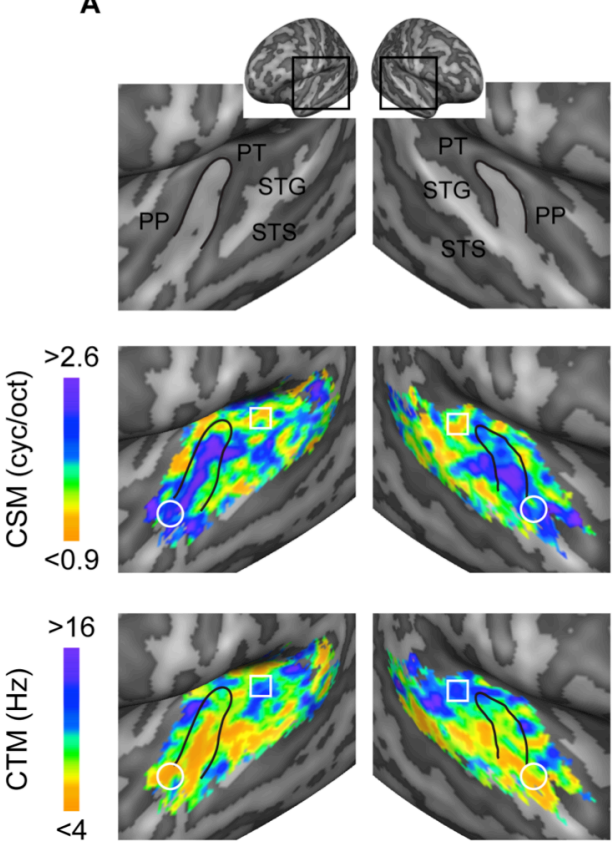

$3 \mathrm{~T}$
B
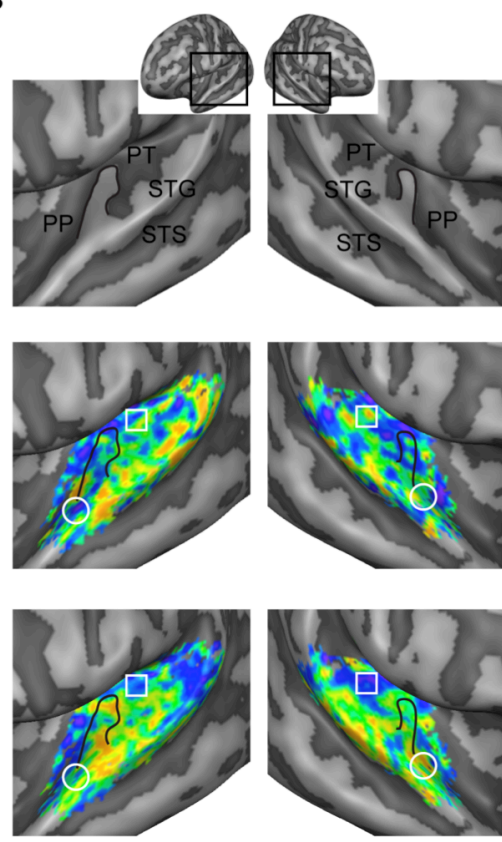

$7 \mathrm{~T}$

Figure 2.6. Group maps of CSM and CTM. Maps are displayed for the 3T (A) and 7T (B) datasets. Top: inflated representation of the group cortex. Maps are shown in the cortical region highlighted by the black square. Middle, Bottom: purple denotes tuning for fine (fast) spectral (temporal) structures; orange denotes tuning for coarse (slow) spectral (temporal) features. The white circle and square outline anterior/ventral and posterior/dorsal auditory regions, respectively. The black line indicates HG. 
CSM

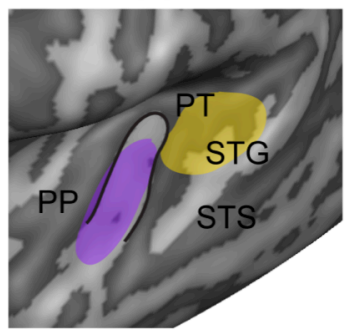

High CSM

Low CSM
CTM

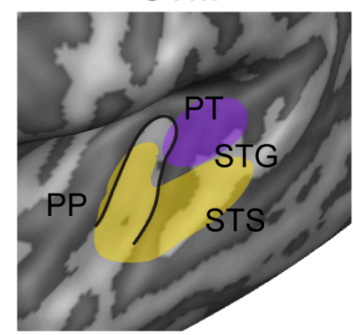

High CTM

Low CTM

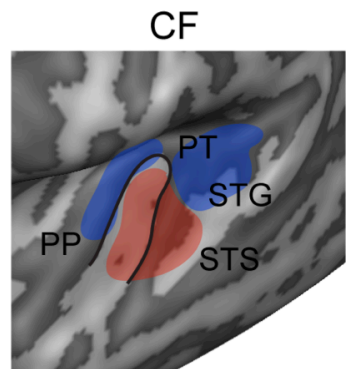

High CF

Low CF

Figure 2.7. Summary of spatial distribution of voxels' tuning properties. The cartoon is a schematic representation of regional preferences for spectral modulation (left), temporal modulation (middle), and frequency (right). The schematic of spectral and temporal modulation preference only summarize the most evident characteristics emerging from the complex spatial pattern of CSM and CTM. The cartoon of frequency preference shows the main tonotopic gradient in regions along and surrounding HG. Cartoon clusters are superimposed over the left hemisphere of the group cortex as derived from the experiment at $3 \mathrm{~T}$. The black line indicates $\mathrm{HG}$.

\section{Discussion}

\section{Mechanisms of spectral and temporal modulation processing}

Our results show that the representation of natural sounds in the human auditory cortex relies on a frequency-specific analysis of combined spectrotemporal modulations. By showing superior performance of the joint MTF-based model over the independent model, we have demonstrated that the hypothesis of independent tuning for spectral (Shamma et al., 1995) and temporal modulations (Jepsen et al., 2008) is insufficient to account for the representation of natural sounds in the human auditory cortex. Furthermore, the frequency-specificity that we revealed indicates that the organization of the auditory cortex according to frequency extends beyond the representation of the spectral content of incoming sounds. We show that, at least for spectro-temporal modulations, the integration along the whole range of frequencies occurs at a later stage than the extraction of the feature itself.

The encoding mechanism that our results support is consistent with a recent study showing that a frequency-specific representation of combined spectro- 
temporal modulations allows the accurate reconstruction of speech in the human posterior superior temporal gyrus (Pasley et al., 2012). The present study generalizes these observations to sounds from natural categories other than speech. Furthermore, our results are in line with psychophysics studies showing that tuning for combined spectro-temporal modulations provides a better account of human behavior during the performance of auditory tasks (Patil et al., 2012; Sabin et al., 2012).

Previous neuroimaging studies had examined the processing of spectral and temporal modulations by measuring the tuning to synthetic stimuli with varying spectral modulation frequency, temporal modulation frequency or the combination of the two. This approach suffers from two main limitations. First, natural sounds are complex stimuli with characteristic statistical regularities (Voss and Clarke, 1975; Attias and Schreiner, 1997; Escabí et al., 2003; Singh and Theunissen, 2003) and it has been suggested that the auditory system is adapted to such regularities in order to efficiently encode sounds in natural settings (Barlow, 1961). Even the most complex synthetic stimuli lack both the statistical structure and the behavioral relevance of natural sounds; therefore there is not guarantee that they engage the auditory cortex in processing that is actually used during the analysis of natural sounds. Second, tuning per se only allows indirect inference on cortical encoding mechanisms: proofing a general computational strategy requires building a model that is able to predict brain responses to a broad range of natural stimuli (Wu et al., 2006). The approach that we followed in the present study allowed overcoming these limitations, therefore providing direct evidence for a specific encoding mechanism. However, two important caveats should be mentioned. First, by estimating a linear mapping between modulation acoustic space and fMRI responses, we only modeled the linear response properties of voxels. One might argue that because of the linear approximation, the use of natural sounds provides no advantage over synthetic stimuli (e.g. dynamic ripples). However, it has been shown that tuning properties of both auditory (Theunissen et al., 2000; Bitterman et al., 2008; Laudanski et al., 2012) and visual (David et al., 2004; Talebi and Baker, 2012) neurons differ significantly under natural and synthetic stimulus condition and that linear models obtained from natural stimuli predict neurons responses significantly better. This shows that natural and synthetic stimuli activate neurons in a different manner and that, despite being an incomplete description, linear models estimated from responses to natural stimuli may be more accurate. We suggest that this is true also for models of voxels receptive fields. Second, it might be possible that some auditory cortical locations are selective to higher-level sound 
attributes (i.e. sound categories) that co-occur with specific spectro-temporal modulations. As a consequence of this co-occurrence, these locations would then be assigned with a preferred temporal and spectral modulation frequency, only in virtue of their category selectivity. To examine the role of category selectivity on our results, we performed additional analyses on the $7 \mathrm{~T}$ dataset and tested a model that included categorical predictors together with the original MTF-based model (Supplementary Methods). The results showed that predictions of new sounds do not improve with the inclusion of categorical information (mean [SE] = 0.76 [0.03]) and that estimated CTM and CSM maps do not change (Figure S2.8). This analysis suggests that category tuning may result from preference to specific lower level features or combination of features. However, it would be important to further investigate this issue and compare responses and voxels receptive fields obtained with both natural and synthetic sounds (see (Moerel et al., 2012) for a similar comparison for frequency responses). Such an investigation is experimentally challenging, as it would require as many stimuli (dynamic ripples) as model parameters used in the present study. However, it could be crucial for understanding the relation between acoustic and perceptual levels of sound representation in the auditory cortex.

\section{Spatial topographies and relation to current functional models}

On the basis of positron emission tomography responses to tone sequences that differed either in the temporal or spectral dimension, Zatorre and Belin (2001) reported a left-hemispheric preference for rapid temporal processing and complementary preference in the right hemisphere for fine-grained spectral analysis. While the analyses we conducted cannot exclude that hemispheric differences exist at regional level, our maps - obtained at a much higher spatial resolution and with natural sounds - suggest a more complex spatial pattern of spectral and temporal modulation preference within each hemisphere. The most evident characteristic is that - in both the hemispheres - regions located posteriorlaterally to HG (see squares in Figure 2.6 and the schematic summary in Figure 2.7) preferably encode coarse spectral information with high temporal precision while regions located along HG or antero-ventrally (see circles in Figure 2.6 and the schematic summary in Figure 2.7) preferably encode fine-grained spectral information with low temporal precision. Both the two previous human neuroimaging studies that investigated tuning for combined spectro-temporal modulations with dynamic ripples (Langers et al., 2003; Schönwiesner and Zatorre, 2009) reported a role of anterior auditory regions in the analysis of fine spectral 
details, which is consistent with our observations, whereas results are less coherent for temporal modulation maps. Again, a direct comparison between maps obtained with dynamic ripples and natural sounds would be required to address this issue.

Our results of spatial topographies for CTM and CTF support the view that the auditory cortex forms multiple (parallel) representations of the incoming sounds at different spectro-temporal resolutions (Bendor and Wang, 2008; Samson et al., 2011). We suggest that this may be relevant for enabling flexible behavior, as different goal-oriented sound processing may benefit from different types of auditory representations. Importantly, this suggestion can be tested empirically in future experiments and studies where (natural) sounds are presented in the context of multiple behavioral tasks.

A spectral-temporal resolution "trade-off" analogous to the one reported here has previously been described for neurons in the inferior colliculus of the cat (Rodríguez et al., 2010a, 2010b) and is in agreement with the low-pass behavior of the MTF of the human auditory cortex (Schönwiesner and Zatorre, 2009) and the psychophysically derived detection thresholds for spectro-temporal modulations (Chi et al., 1999). Furthermore, modulation spectra of natural sounds exhibit a similar trade-off, i.e. natural sounds rarely present both high spectral and high temporal modulation frequencies (Singh and Theunissen, 2003; Elliott and Theunissen, 2009). A match between stimulus statistics and neuronal response properties is generally interpreted as an evidence for the theory of efficient coding (Barlow, 1961; Simoncelli and Olshausen, 2001; Escabí et al., 2003; Woolley et al., 2005; Rodríguez et al., 2010a). Thus, our data provide further support to the idea that the auditory system has adapted in order to efficiently encode the statistical regularities of natural sounds.

\section{Comparing computational models of auditory processing with $\mathrm{fMRI}$}

Besides providing insights into the representation of natural sounds in the human auditory cortex, our results pave the way to future research aiming at testing increasingly complex encoding models of auditory processing. The combination of fMRI and "encoding" techniques has proven to be a successful tool to investigate the representation of natural images in the human visual cortex (Kay et al., 2008b; Naselaris et al., 2009; Nishimoto et al., 2011), as well as to predict the brain activity associated with the meaning of words (Mitchell et al., 2008). In the auditory domain, the application of such powerful method has lagged behind. We 
have recently demonstrated that "encoding" makes it possible to detect the spectral tuning of voxels in the human auditory cortex from fMRI responses to natural sounds (Moerel et al., 2012, 2013; De Martino et al., 2013). In the present study, we show that models embedding more complex representations than frequency selectivity can be learned from fMRI activity. The challenge for future studies is to explore more sophisticated voxels receptive field models. Here we only considered voxels tuning along three stimulus dimensions (frequency, spectral modulations and temporal modulations). However, natural sounds vary in a higher dimensional acoustic space and interactions with parameters not considered here might occur.

Interestingly, we consistently observed higher prediction accuracy for the 7T compared to the $3 \mathrm{~T}$ dataset (Figure 2.4), despite the fact that at $7 \mathrm{~T}$ the model was trained and tested on independent sound ensembles (while different presentations of the same sounds were used for the $3 \mathrm{~T}$ data set). We interpret this difference as a result of the interplay between two important factors, namely the number of stimuli and the functional contrast to noise ratio (CNR). The larger amount of different sounds employed in the $7 \mathrm{~T}$ experiment has probably increased the variance along the dimensions represented by the model; this, together with the higher CNR and the higher spatial specificity achieved at 7T, has likely led to a more accurate model estimation, which in turn has resulted in higher prediction accuracy. These observations provide important guidelines for the design of future experiments in this framework.

It should be mentioned that in our study, accuracy based on percent correct was significantly above chance ([12.5\%, 12.5\%, 16.7\%, 20.8\%, 25\%] for subjects S6S10 for the best performing model at $7 \mathrm{~T}$; chance $=4.2 \%$ ), but still quite small compared to the outstanding results reported in similar encoding studies in the visual domain (e.g. (Kay et al., 2008b)). However, the distribution of ranks was skewed towards 1 (correct identification), indicating that for most sounds the correlation between predicted and measured response was ranked very high (e.g. second or third). The lower percent correct performance for sound identification can be ascribed to a variety of reasons. It might be due to the lower functional CNR, as BOLD responses observed in the auditory cortex are substantially lower than those in the visual cortex, probably because of the effects of the scanner noise (Gaab et al., 2007). Furthermore, our clustered fMRI acquisition with a silent gap between scans limits the number of sounds used for training/testing the model (compared e.g. to the number of images in (Kay et al., 2008b)). Finally, the model of receptive field based on spectro-temporal modulations might be too simple for 
allowing distinguishing two acoustically similar sounds (e.g. two speech sounds).

Although the proposed combination of high field $\mathrm{fMRI}$ with the encoding approach is valuable for testing well-defined hypotheses on sound processing in the human brain, there are intrinsic limitations. A voxel - even at the high spatial resolution achievable with $7 \mathrm{~T} \mathrm{fMRI}$ - samples a large number of neurons and the relation between the measured BOLD signal and the neural activation is only partly understood. Results based on BOLD fMRI (and thus fMRI encoding) reflect a complex mixture of neuronal (spiking and synaptic activity, excitation, inhibition) as well as neurovascular phenomena. In particular, neural inhibition may be associated with both positive and negative BOLD, depending on the specific neural network configuration (Logothetis, 2008). Understanding the neuronal dynamics underlying our fMRI observations would thus require combining electrophysiological (at single-cell and neuronal population level) and fMRI investigations in animal models (Logothetis et al., 2001) and/or humans (Bitterman et al., 2008).

In summary, our study represents a first demonstration of how fMRI data and "encoding" techniques can be successfully combined to test competing computational models of auditory processing and to concurrently estimate response properties of cortical locations along multiple dimensions within an ecologically valid framework. Also, by using a biologically inspired computational model, we pave the way for linking electrophysiology in animals and non-invasive research in humans.

\section{Materials and Methods}

\section{Ethics Statement}

The Ethical Committee of the Faculty of Psychology and Neuroscience at Maastricht University and the Institutional Review Board for human subject research at the University of Minnesota granted approval for the study at 3T and 7T respectively.

\section{Experimental procedure}

Subjects, stimuli, experimental design, MRI parameters, and data preprocessing have been reported in previous publications from our group (Moerel et al., 2012, 2013; De Martino et al., 2013) (see Supplementary Methods). In the following, the most relevant details of the experimental design will be briefly 
described.

We used 60 (168) recordings of natural sounds for the 3T (7T) experiment. Stimuli included human vocal sounds (both speech and non-speech, e.g., baby cry, laughter, coughing), animal cries (e.g., dog, cat, horse), musical instruments (e.g., piano, flute, drums), scenes from nature (e.g., rain, wind, thunder), and tool sounds (e.g., keys, scissors, vacuum cleaner). Sounds were sampled at $16 \mathrm{kHz}$ and their duration was cut at $1000 \mathrm{~ms}$. Sound onset and offset were ramped with a $10 \mathrm{~ms}$ linear slope, and their energy (RMS) levels were equalized.

The 3T and 7T experiments consisted of 3 and 8 runs, respectively; in the 3T (7T) experiment, each run lasted approximately 25 (10) minutes. In the 7T experiment, data were subdivided into six train runs and two test runs. In the train runs, 144 of the 168 stimuli were presented with 3 repetitions overall (i.e. each sound was presented in 3 of the 6 train runs). The remaining 24 sounds were presented in the test runs and repeated 3 times per run.

Sounds were presented in the silent gap between acquisitions with a randomly assigned inter-stimulus interval of 2, 3, or 4 TRs - plus an additional random jitter. Zero trials (trials where no sound was presented; $10 \%$ of the trials in the $3 \mathrm{~T}$ experiment; $6 \%$ (5\%) of the trials in train (test) runs in the $7 \mathrm{~T}$ experiment), and catch trials (trials in which the sound which was just heard was presented; $6 \%$ of the trials in the 3T experiment; $6 \%(3 \%)$ of the trials in train (test) runs in the 7T experiment) were included. Subjects responded with a button press when a sound was repeated. Catch trials were excluded from the analysis.

Joint frequency-specific MTF-based model

The stimulus representation in the modulation space was obtained as the output of a biologically inspired model of auditory processing (Chi et al., 2005), which explicitly encodes the modulation content of a sound spectrogram. The auditory model consists of two main components: an early stage that accounts for the transformations that acoustic signals undergo in the early auditory system, from the cochlea to the midbrain; and a cortical stage that simulates the processing of the acoustic input at the level of the (primary) auditory cortex (see Chapter 1 for a detailed description of the model).

We derived the auditory spectrogram and its modulation content using the "NSLTools" package (available at http://www.isr.umd.edu/Labs/NSL/Software.htm) and customized Matlab code (The MathWorks Inc.). Sounds' spectrograms were generated using a bank of 128 overlapping bandpass filters with constant- $Q\left(Q_{10 d B}\right.$ 
= 3) mimicking the auditory periphery. Filters were equally spaced along a logarithmic frequency axis and covered a range of 5.3 oct $(f=180-7040 \mathrm{~Hz})$. The output of the filter bank underwent band-pass filtering (hair cell stage), first-order derivative with respect to the frequency axis, half-wave rectification, and short-term temporal integration with time constant $\tau=8 \mathrm{~ms}$ (midbrain stage). Pilot analyses showed that model performance was not significantly affected by changes in the parameters of the early stage. Accordingly, parameters for the spectrogram estimation were fixed (i.e. not estimated in the fitting procedure) and set as described in (Chi et al., 2005). The modulation content of the auditory spectrogram was computed through a bank of 2D modulation selective filters tuned to spectral modulation frequencies of $\Omega=[0.5,1,2,4]$ cycloct and temporal modulation frequencies of $\omega=[1,3,9,27] \mathrm{Hz}$. The filter bank output was computed at each frequency along the tonotopic axis and then averaged over time. In order to avoid overfitting, a reduced modulation representation was obtained as follows (3T: 3 tonotopic frequencies $\times 4$ spectral modulations $\times 4$ temporal modulations $=48$ parameters to learn; 7T: 8 tonotopic frequencies $\times 4$ spectral modulations $\times 4$ temporal modulations $=128$ parameters to learn; note that we chose a different number of parameters for the $3 \mathrm{~T}$ and $7 \mathrm{~T}$ datasets due to the different number of stimuli used for model's estimation - 60 and 144 stimuli, respectively). First, the time-averaged output of the filter bank was averaged across the upward and downward filter directions (note that this corresponds to assuming that sweep direction does not affect voxels activation levels). Then, we divided the tonotopic axis in ranges with constant bandwidth in octaves and averaged the modulation energy within each of these regions. We defined three frequency ranges in the 3T experiment and eight in the $7 \mathrm{~T}$ experiment. The above processing steps were applied to all stimuli, resulting into an $[S \times N]$ feature matrix $\mathbf{F}$ of average modulation energy, where $S$ is the number of sounds, and $N$ is the number of features in the reduced modulation representation.

\section{Tonotopy model}

The stimuli representation in the frequency space was obtained using only the input stage of the auditory model. The spectrogram was computed at 128 logarithmically spaced frequency values $(f=180-7040 \mathrm{~Hz})$ and averaged over time. In the $3 T$ experiment, we generated a reduced frequency representation in order to restrain the effects of overfitting (note that in the 7T experiment the number of observations in the train set was already higher than the number of parameters to estimate). We divided the tonotopic axis in 48 bins with constant bandwidth in 
octaves and averaged the frequency content within each of these regions. We chose 48 bins in order to have the same number of parameters for both the MTFbased and the tonotopy model. The above processing steps were applied to all stimuli, resulting into an $[S \times N]$ feature matrix $\mathbf{F}$ of time-averaged frequency content, where $S$ is the number of sounds, and $N$ is the number of frequency bins.

\section{Joint frequency non-specific MTF-based model}

We generated the non-localized modulation representation by averaging the frequency-specific joint representation along both time and frequency (this is similar to performing a 2D Fourier transform of the spectrogram). This resulted in a representation with 16 features (4 temporal modulations $\times 4$ spectral modulations). However, frequency specific information is indeed reflected in voxels' activity (Formisano et al., 2003; Moerel et al., 2012); therefore, we concatenated the modulation representation with a tonotopic representation obtained as described above for the tonotopy model. We employed 32 frequency bins for the $3 T$ dataset and 112 for the 7T dataset, resulting in a final representation with 48 and 128 features, respectively.

\section{Independent frequency-specific MTF-based model}

We generated the independent modulation representation by filtering the auditory spectrogram with one-dimensional purely spectral and purely temporal modulation filters. Filters were tuned to spectral modulation frequencies of $\Omega=$ $[0.5,1,2,4]$ cyc/oct and temporal modulation frequencies of $\omega=[1,3,9,27] \mathrm{Hz}$. The output of each filter bank was averaged over time and within frequency ranges with constant bandwidth in octaves. In order to have a representation with the same number of features as for the joint model, we defined 6 frequency ranges in the $3 \mathrm{~T}$ experiment and 16 in the $7 \mathrm{~T}$ experiment. Finally, the outputs of the purely spectral and purely temporal filter banks were concatenated, resulting in a representation with 48 features for the $3 T$ dataset (6 tonotopic frequencies $\times 4$ temporal modulations +6 tonotopic frequencies $\times 4$ spectral modulations) and 128 for the $7 \mathrm{~T}$ dataset (16 tonotopic frequencies $\times 4$ temporal modulations +16 tonotopic frequencies $\times 4$ spectral modulations). The above processing steps were applied to all stimuli, producing an $[S \times N]$ feature matrix $\mathbf{F}$ of average modulation energy, where $S$ is the number of sounds, and $N$ is the number of features. 
Independent frequency non-specific MTF-based model

We generated the non-localized independent representation by averaging across frequency the frequency-specific independent representation. This resulted in a representation with 8 features $(4$ temporal modulations +4 spectral modulations). The final model was obtained by concatenating the modulation representation with a tonotopic representation obtained as described above for the tonotopy model. We employed 40 frequency bins for the $3 \mathrm{~T}$ dataset and 120 for the 7T dataset, resulting in a final representation with 48 and 128 features, respectively.

\section{Model estimation and evaluation}

In the 7T experiment, independent train and test runs involving two completely distinct sound sets were used to train and assess the model, whereas leave run out cross-validation was performed for the $3 T$ dataset (the final model parameters and the overall prediction accuracy were computed as the average across cross validations).

\section{Estimation of $\mathrm{fMRI}$ responses to natural sounds}

For each voxel $i$, the response vector $Y_{i}[(S \times 1), S=$ number of sounds] was obtained in two steps. First, a deconvolution analysis with all stimuli treated as a single condition was used to estimate the hemodynamic response function (HRF) common to all stimuli. Then, using this HRF and one predictor per sound, we computed the beta weight of each sound (Kay et al., 2008a). Further analyses were performed on voxels with a significant response to the sounds ( $p<.05$, uncorrected in order not to be too stringent at this stage of the process) within an anatomically defined mask, which included HG, PT, PP, and STG.

\section{Estimation of model parameters}

The $\mathrm{fMRI}$ activity $Y_{i}\left[S_{\text {train }} \times 1\right]$ at voxel $i$ was modeled as a linear transformation of the feature matrix $\mathbf{F}_{\text {train }}\left[S_{\text {train }} \times N\right]$ plus a noise term $n\left[S_{\text {train }} \times 1\right]$ as follows:

$$
Y_{i}=\mathbf{F}_{\text {train }} C_{i}+n
$$

where $S_{\text {train }}$ is the number of sounds in the training set, and $C_{i}$ is an $[N \times 1]$ vector of model parameters, whose elements $c_{i j}$ quantify the contribution of feature $j$ to the overall response of voxel $i$. Note that Equation 1 does not include a constant term 
as columns of matrices $\mathbf{F}_{\text {train }}$ and $Y_{i}$ were converted to standardized z-scores. Zscoring of the features and responses does not affect the expressive capacity of the linear regression model. However, in a regularized regression framework like ridge regression (see below), z-scoring does affect the estimated model parameters (weights). In the present study, z-score was performed because the energy content of natural sounds varies on different scales across frequencies and modulations. As a consequence, the estimated model parameters would not be comparable without performing the z-score normalization.

The solution to Equation 1 was computed using ridge regression (Hoerl and Kennard, 1970a). The regularization parameter $\lambda$ was determined independently for each voxel by automatically inspecting the stability of the ridge trace, that is changes in the parameter estimates as a function of $\lambda$ (Hoerl and Kennard, 1970b).

Namely, parameter estimates $\tilde{c} i j(\lambda)$ were obtained for a range of increasing $\lambda$ values $\left[\lambda_{1}, \lambda_{2}, \ldots, \lambda_{p}\right]$, and the regularization parameter was set at the value $\lambda^{*}$ where all parameter estimates consistently changed less than $20 \%$ of their initial value $\tilde{c}_{i j}\left(\lambda_{1}\right)$ :

$$
\lambda^{*}=\lambda: \quad \frac{\partial \tilde{c}_{i j}(\lambda)}{\partial \lambda}<0.2 \tilde{c}_{i j}\left(\lambda_{1}\right) \quad \forall \lambda \geq \lambda^{*}, \forall \tilde{c}_{i j}(\lambda)
$$

The inspection of the ridge trace represented an advantage in terms of trade-off between accurate model estimation and computational load. Namely, we observed that the selection of the regularization parameter via cross validation was computationally slower, while not yielding any significant improvement on models performance.

Model evaluation

We quantified model's prediction accuracy by performing a sound identification analysis (Kay et al., 2008b). Namely, we used the fMRI activity patterns predicted by the estimated models to identify which sound had been heard among all sounds in the test set.

Because model parameters were estimated in z-score units, we converted to standardized z-score the columns of the feature and response matrices for the stimuli in the test set. Given the trained model $\tilde{\mathbf{C}}[N \times V]$ (where $V$ is the number of voxels), and the feature matrix $\mathbf{F}_{\text {test }}\left[S_{\text {test }} \times N\right]$ for the test set, the predicted fMRI 
activity $\hat{\mathbf{Y}}_{\text {test }}\left[S_{\text {test }} \times \mathrm{V}\right]$ for the test sounds was obtained as follows:

$$
\hat{\mathbf{Y}}_{\text {test }}=\mathbf{F}_{\text {test }} \tilde{\mathbf{C}}
$$

Then, for each stimulus $s_{i}$ we computed the correlation between its predicted fMRI activity $\hat{Y}_{\text {test }}\left(s_{i}\right)\left[1 \times \mathrm{V}\right.$ and all measured fMRI responses $Y_{\text {test }}\left(s_{j}\right)[1 \times \mathrm{V}, \mathrm{j}=$ $1,2, \ldots$, S. The rank of the correlation between predicted and observed activity for stimulus $s_{i}$ was selected as a measure of the model's ability to correctly match $Y_{\text {test }}\left(s_{i}\right)$ with its prediction $\hat{Y}_{\text {test }}\left(s_{i}\right)$. The matching score $m$ for stimulus $s_{i}$ was obtained by normalizing the computed rank between 0 and 1 as follows $(m=1$ indicates correct match; $m=0$ indicates predicted activity pattern for stimulus $s_{i}$ was least similar to the measured one among all stimuli):

$$
m\left(s_{i}\right)=1-\frac{\operatorname{rank}\left(s_{i}\right)-1}{S_{\text {test }}-1}
$$

Normalized ranks were computed for all stimuli in the test set, and the overall model's accuracy was obtained as the mean of the matching scores across stimuli. Note that the metric we used (Eq. 4) generalizes the more straightforward percent correct, a rank-based metric that considers only stimuli that are ranked first, i.e. stimuli that are correctly identified. Percent correct is a comprehensive metric when models identify new stimuli with high accuracies (close to 100\%). As this was not the case in our data (see Discussion), it is informative to look at the whole distribution to assess the degree of incorrect identification.

Statistical significance of the observed accuracy was assessed with permutation testing. Specifically, the empirical null-distribution of accuracies was obtained by randomly permuting ( $P=200$ permutations) the stimulus labels (i.e. $S$ in matrix $\mathbf{Y}$ ) and repeating the training and testing procedures. In order to preserve the spatial correlations among cortical locations, the same permutations were applied to all voxels. The regularization parameter was constant across permutations and was set to the value derived when the model was estimated on the unpermuted set of responses. When compared by means of t-test, accuracies were converted to z-scores via Fisher's transformation in order to reduce deviations from normality. 
Topographic maps of temporal modulation, spectral modulation, and frequency preference

For all voxels, response profiles for temporal modulation, spectral modulation and frequency were computed as marginal sums of the estimated stimulus-activity mapping function $\mathbf{C}$ of the joint frequency-specific MTF-based model, as follows:

$$
\begin{aligned}
& t \operatorname{MTF}(\omega)=\sum_{f} \sum_{\Omega} \mathbf{C}(\omega, \Omega, f) \\
& \operatorname{sMTF}(\Omega)=\sum_{f} \sum_{\omega} \mathbf{C}(\omega, \Omega, f) \\
& f T F(f)=\sum_{\omega} \sum_{\Omega} \mathbf{C}(\omega, \Omega, f)
\end{aligned}
$$

where tMTF and sMTF are the temporal and spectral modulation transfer functions, respectively, and fTF is the frequency transfer function. Voxels characteristic values (CTM, CSM, CF) were defined as the point of maximum of the tMTF, sMTF and fTF, respectively. A continuous representation of preferred values was obtained by spatial smoothing using a 2-neighbor (3-neighbor) voxels filter for the 3T (7T) dataset. Cortical maps were generated by color-coding the voxels' preferred values and projecting them onto an inflated representation of the subject's cortex. Individual maps were subsequently transformed to functional cortex based aligned (fCBA) space (see below) where group maps were obtained as the mean across subjects. Only voxels that had been included in the analysis of at least 3 out of the 5 subjects were considered when computing group maps.

To assess the reliability of the estimated voxels tuning preference, we computed the signal-to-noise ratio (SNR) of the MTFs estimates via a bootstrap resampling procedure applied to all individual subjects (see Supplementary Methods and Figure S2.7).

Relation between voxels characteristic spectral and temporal modulation

For each subject, we computed the Spearman's rank correlation coefficient between voxels characteristics CSM and CTM (prior to spatial smoothing). In order to take into account any possible bias introduced by the model's estimation procedure, we derived the empirical expected value of no correlation by computing the correlation coefficient between voxels CSM and CTM as obtained after permuting the stimulus labels (see above). Statistical significance of the 
Fisher-transformed correlation coefficients was assessed via a group level random effect two-tailed t test.

Functional cortex based alignment

Additionally to the main experiments, localizer data were collected as responses to amplitude modulated tones (see Supplementary Methods). Tonotopy maps were computed with best-frequency mapping (Formisano et al., 2003), and resulting maps were used for fCBA (Goebel et al., 2006) as follows. In each subject and hemisphere, we delineated the low frequency region consistently present in the vicinity of Heschl's gyrus as region of interest. FCBA was partially driven by this functional region (weighting decreased over iterations), and partially by anatomical information (weighting increased over iterations; (Frost and Goebel, 2013)). The resulting alignment information was used for calculating and displaying group cortical maps.

\section{Acknowledgments}

We thank G. Valente and L. Hausfeld for valuable discussions. This work was supported by Maastricht University and the Netherlands Organization for Scientific Research (NWO grants 22-001-036, 453-12-002, 021-002-102), the National Institutes of Health (NIH grants P41 EB015894, P30 NS076408, and S10 RR26783), and the WM KECK Foundation.

\section{References}

Ahveninen J, Jääskeläinen IP, Raij $T$, Bonmassar $G$, Devore $S$, Hämäläinen $M$, Levänen S, Lin F-H, Sams M, Shinn-Cunningham BG, Witzel T, Belliveau JW (2006) Task-modulated "what" and "where" pathways in human auditory cortex. Proc Natl Acad Sci U S A 103:14608-14613.

Alain C, Arnott SR, Hevenor S, Graham S, Grady CL (2001) "What" and "where" in the human auditory system. Proc Natl Acad Sci U S A 98:12301-12306.

Attias H, Schreiner CE (1997) Temporal Low-Order Statistics of Natural Sounds Mozer MC, Jordan MI, Petsche T, eds. Adv Neural Inf Process Syst 9:27-33.

Barlow H (1961) Possible principles underlying the transformation of sensory messages. Sens Commun:217-234. 
Barton B, Venezia J (2012) Orthogonal acoustic dimensions define auditory field maps in human cortex. Proc Natl Acad Sci U S A 109:20738-20743.

Belin P, Zatorre RJ, Lafaille P, Ahad P, Pike B (2000) Voice-selective areas in human auditory cortex. Nature 403:309-312.

Bendor D, Wang X (2008) Neural response properties of primary, rostral, and rostrotemporal core fields in the auditory cortex of marmoset monkeys. J Neurophysiol 100:888-906.

Bitterman Y, Mukamel R, Malach R, Fried I, Nelken I (2008) Ultra-fine frequency tuning revealed in single neurons of human auditory cortex. Nature 451:197201.

Chi T, Gao Y, Guyton MC, Ru P, Shamma S (1999) Spectro-temporal modulation transfer functions and speech intelligibility. J Acoust Soc Am 106:2719-2732.

Chi T, Ru P, Shamma S (2005) Multiresolution spectrotemporal analysis of complex sounds. J Acoust Soc Am 118:887-906.

David S V, Vinje WE, Gallant JL (2004) Natural stimulus statistics alter the receptive field structure of v1 neurons. J Neurosci 24:6991-7006.

De Martino F, Moerel M, van de Moortele P-F, Ugurbil K, Goebel R, Yacoub E, Formisano E (2013) Spatial organization of frequency preference and selectivity in the human inferior colliculus. Nat Commun 4:1386.

Depireux DA, Simon JZ, Klein DJ, Shamma SA, Ding N (2001) Spectro-Temporal Response Field Characterization With Dynamic Ripples in Ferret Primary Auditory Cortex. J Neurophysiol 85:1220-1234.

Drullman R, Festen J, Plomp R (1994) Effect of temporal envelope smearing on speech reception. J Acoust Soc Am 95:1053-1064.

Elhilali M, Shamma S (2008) A cocktail party with a cortical twist: how cortical mechanisms contribute to sound segregation. J Acoust Soc Am 124:37513771.

Elliott TM, Theunissen FE (2009) The modulation transfer function for speech intelligibility. PLoS Comput Biol 5:e1000302. 
Escabí MA, Miller LM, Read HL, Schreiner CE (2003) Naturalistic auditory contrast improves spectrotemporal coding in the cat inferior colliculus. J Neurosci 23:11489-11504.

Formisano E, Kim DS, Di Salle F, van de Moortele PF, Ugurbil K, Goebel R (2003) Mirror-symmetric tonotopic maps in human primary auditory cortex. Neuron 40:859-869.

Frost MA, Goebel R (2013) Functionally informed cortex based alignment: An integrated approach for whole-cortex macro-anatomical and ROI-based functional alignment. Neuroimage 83:1002-1010.

Gaab N, Gabrieli JDE, Glover GH (2007) Assessing the influence of scanner background noise on auditory processing. I. An fMRI study comparing three experimental designs with varying degrees of scanner noise. Hum Brain Mapp 28:703-720.

Giraud AL, Lorenzi C, Ashburner J, Wable J, Johnsrude I, Frackowiak R, Kleinschmidt A (2000) Representation of the temporal envelope of sounds in the human brain. J Neurophysiol 84:1588-1598.

Goebel R, Esposito F, Formisano E (2006) Analysis of functional image analysis contest (FIAC) data with brainvoyager QX: From single-subject to cortically aligned group general linear model analysis and self-organizing group independent component analysis. Hum Brain Mapp 27:392-401.

Green D (1986) "Frequency" and the Detection of Spectral Shape Change. In: Auditory Frequency Selectivity (Moore BJ, Patterson R, eds), pp 351-359 Nato ASI Series. Springer US.

Hoerl AE, Kennard RW (1970a) Ridge Regression: Biased Estimation for Nonorthogonal Problems. Technometrics 12:55-67.

Hoerl AE, Kennard RW (1970b) Ridge Regression: Applications to Nonorthogonal Problems. Technometrics 12:69-82.

Jepsen ML, Ewert SD, Dau T (2008) A computational model of human auditory signal processing and perception. J Acoust Soc Am 124:422-438.

Joris PX, Schreiner CE, Rees A (2004) Neural processing of amplitude-modulated sounds. Physiol Rev 84:541-577. 
Kay KN, David S V, Prenger RJ, Hansen KA, Gallant JL (2008a) Modeling lowfrequency fluctuation and hemodynamic response timecourse in eventrelated fMRI. Hum Brain Mapp 29:142-156.

Kay KN, Naselaris T, Prenger RJ, Gallant JL (2008b) Identifying natural images from human brain activity. Nature 452:352-355.

Kowalski N, Depireux DA, Shamma SA (1996) Analysis of dynamic spectra in ferret primary auditory cortex. I. Characteristics of single-unit responses to moving ripple spectra. J Neurophysiol 76:3503-3523.

Langers DRM, Backes WH, Dijk P Van (2003) Spectrotemporal features of the auditory cortex: the activation in response to dynamic ripples. Neuroimage 20:265-275.

Laudanski J, Edeline J-M, Huetz C (2012) Differences between Spectro-Temporal Receptive Fields Derived from Artificial and Natural Stimuli in the Auditory Cortex. PLoS One 7:e50539.

Leaver AM, Rauschecker JP (2010) Cortical representation of natural complex sounds: effects of acoustic features and auditory object category. J Neurosci 30:7604-7612.

Lewis JW, Talkington WJ, Walker NA, Spirou GA, Jajosky A, Frum C, BrefczynskiLewis J a (2009) Human cortical organization for processing vocalizations indicates representation of harmonic structure as a signal attribute. J Neurosci 29:2283-2296.

Logothetis NK (2008) What we can do and what we cannot do with fMRI. Nature 453:869-878.

Logothetis NK, Pauls J, Augath M, Trinath T, Oeltermann A (2001) Neurophysiological investigation of the basis of the fMRI signal. Nature 412:150-157.

Mitchell TM, Shinkareva S V, Carlson A, Chang K-M, Malave VL, Mason R a, Just MA (2008) Predicting human brain activity associated with the meanings of nouns. Science 320:1191-1195.

Moerel M, De Martino F, Formisano E (2012) Processing of natural sounds in human auditory cortex: tonotopy, spectral tuning, and relation to voice sensitivity. J Neurosci 32:14205-14216. 
Moerel M, De Martino F, Santoro R, Ugurbil K, Goebel R, Yacoub E, Formisano E (2013) Processing of natural sounds: characterization of multipeak spectral tuning in human auditory cortex. J Neurosci 33:11888-11898.

Naselaris T, Prenger RJ, Kay KN, Oliver M, Gallant JL (2009) Bayesian reconstruction of natural images from human brain activity. Neuron 63:902915.

Nishimoto S, Vu AT, Naselaris T, Benjamini Y, Yu B, Gallant JL (2011) Reconstructing Visual Experiences from Brain Activity Evoked by Natural Movies. Curr Biol:1641-1646.

Pasley BN, David S V, Mesgarani N, Flinker A, Shamma S, Crone NE, Knight RT, Chang EF (2012) Reconstructing Speech from Human Auditory Cortex Zatorre R, ed. PLoS Biol 10:e1001251.

Patil K, Pressnitzer D, Shamma S, Elhilali M (2012) Music in our ears: the biological bases of musical timbre perception. PLoS Comput Biol 8:e1002759.

Rodríguez FA, Chen C, Read HL, Escabí MA (2010a) Neural modulation tuning characteristics scale to efficiently encode natural sound statistics. J Neurosci 30:15969-15980.

Rodríguez FA, Read HL, Escabí MA (2010b) Spectral and temporal modulation tradeoff in the inferior colliculus. J Neurophysiol 103:887-903.

Sabin AT, Eddins DA, Wright BA (2012) Perceptual Learning Evidence for Tuning to Spectrotemporal Modulation in the Human Auditory System. J Neurosci 32:6542-6549.

Samson F, Zeffiro TA, Toussaint A, Belin P (2011) Stimulus complexity and categorical effects in human auditory cortex: an activation likelihood estimation meta-analysis. Front Psychol 1 .

Schönwiesner M, Zatorre RJ (2009) Spectro-temporal modulation transfer function of single voxels in the human auditory cortex measured with high-resolution fMRI. Proc Natl Acad Sci U S A 106:14611-14616.

Schreiner CE, Winer JA (2007) Auditory cortex mapmaking: principles, projections, and plasticity. Neuron 56:356-365. 
Shamma S, Versnel H, Kowalski N (1995) Ripple Analysis in Ferret Primary Auditory Cortex. I. Response Characteristics of Single Units to Sinusoidally Rippled Spectra. Aud Neurosci 1:233-254.

Shannon R, Zeng F, Kamath $V(1995)$ Speech recognition with primarily temporal cues. Science (80- ) 270:303-304.

Simoncelli EP, Olshausen BA (2001) Natural Image Statistics and Neural Representation. Annu Rev Neurosci 24:1193-1216.

Singh NC, Theunissen FE (2003) Modulation spectra of natural sounds and ethological theories of auditory processing. J Acoust Soc Am 114:3394-3411.

Talebi V, Baker CL (2012) Natural versus Synthetic Stimuli for Estimating Receptive Field Models: A Comparison of Predictive Robustness. J Neurosci 32:15601576.

Theunissen E, Sen K, Doupe AJ (2000) Spectral-Temporal Receptive Fields of Nonlinear Auditory Neurons. J Neurosci 20:2315-2331.

Viemeister NF (1979) Temporal modulation transfer functions based upon modulation thresholds. J Acoust Soc Am 66:1364-1380.

Voss RF, Clarke J (1975) '1/f noise' in music and speech. Nature 258:317-318.

Woolley SMN, Fremouw TE, Hsu A, Theunissen FE (2005) Tuning for spectrotemporal modulations as a mechanism for auditory discrimination of natural sounds. Nat Neurosci 8:1371-1379.

Wu MC-K, David S V, Gallant JL (2006) Complete functional characterization of sensory neurons by system identification. Annu Rev Neurosci 29:477-505.

Zatorre RJ, Belin P (2001) Spectral and Temporal Processing in Human Auditory Cortex. Cereb Cortex $11: 946-953$. 


\section{Supporting Information}
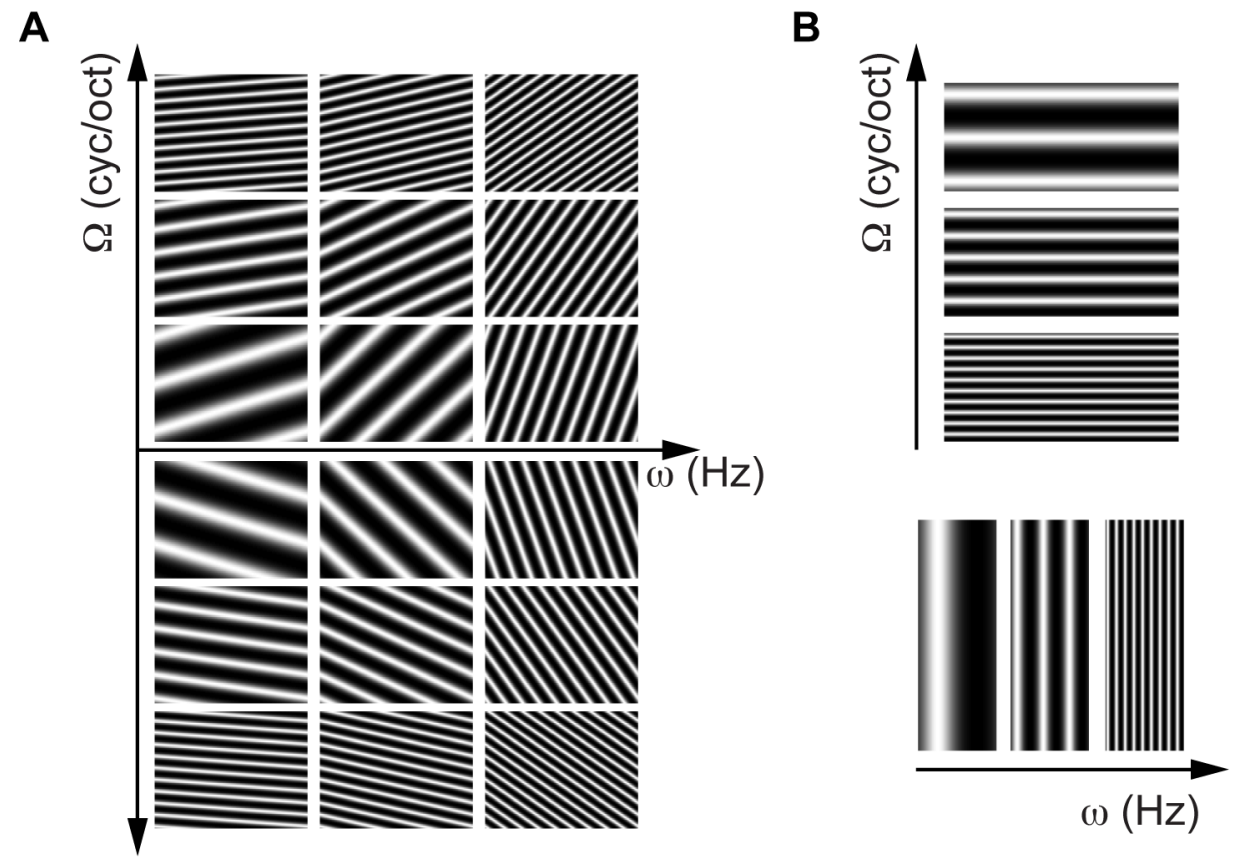

Figure S2.1. Joint and independent modulation representations. Spectrograms illustrate a schematic of channels in a modulation filter bank. Vertical and horizontal spacing between bars indicate channels preferred spectral $(\Omega)$ and temporal modulation frequencies $(\omega)$, respectively. (A) In the joint representation, the conjunction of spectral and temporal modulations is analyzed by spectro-temporal channels tuned to specific combinations of spectral and temporal modulation frequencies. Direction of bar tilt indicates tuning for upward or downward modulations. (B) In the independent representation, spectral and temporal modulations are independently encoded by separate spectral (top) and temporal (bottom) channels. 
A

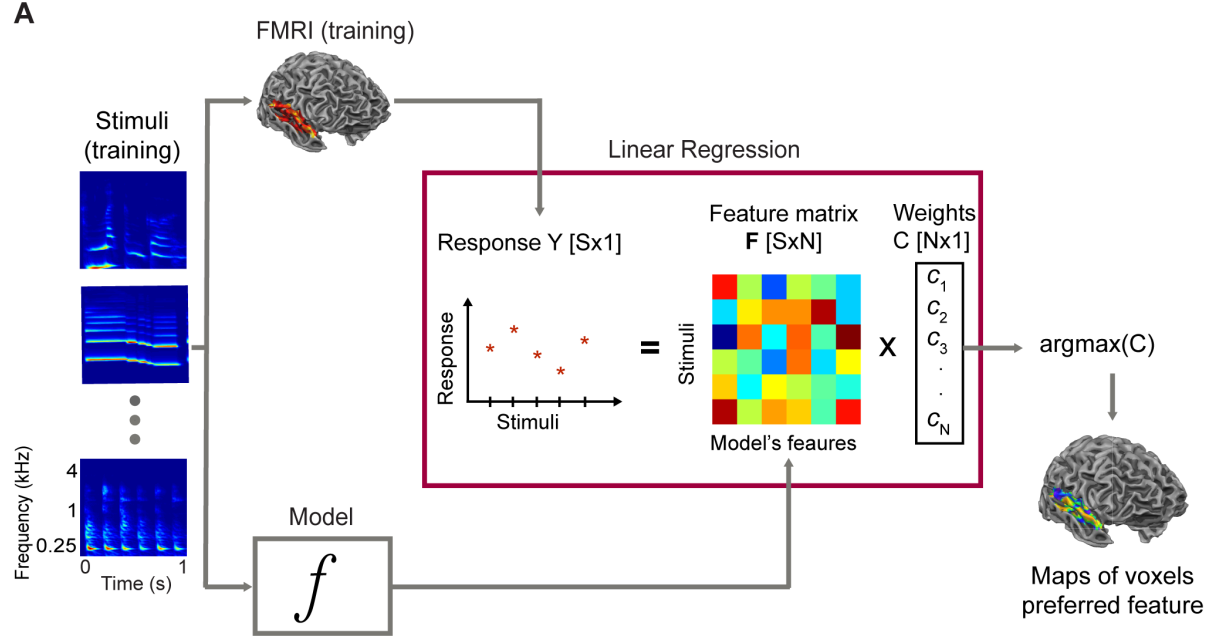

B

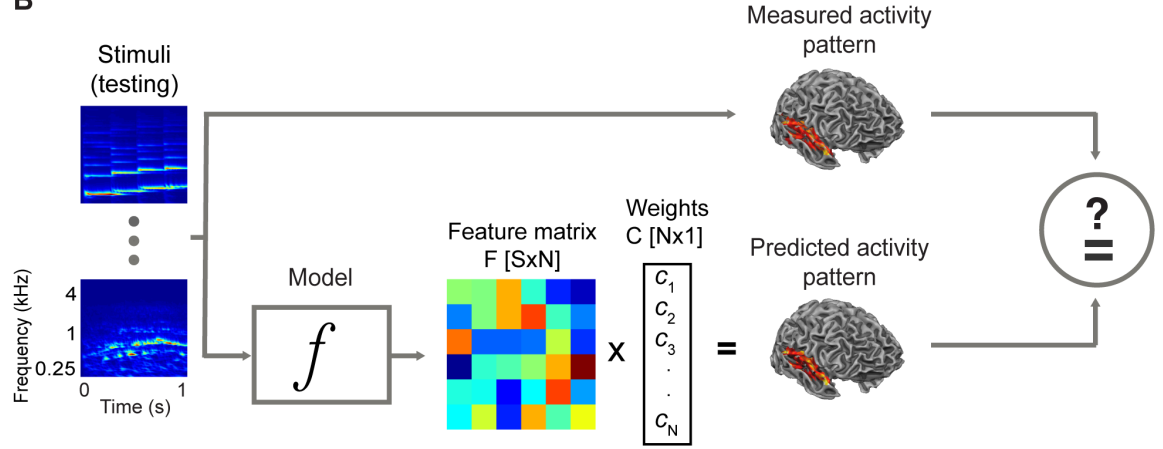

Figure S2.2. Schematic of model estimation and evaluation. (A) FMRI responses to a wide variety of natural sounds are used to estimate an encoding model for each voxel. The model projects the stimuli into an $\mathrm{N}$-dimensional feature space and voxels are described as linear combinations of these features. By applying regularized regression, a vector of model's weights is estimated for each voxel. The feature yielding the highest weight is assigned as voxel's characteristic value. (B) Model performance is evaluated by assessing its ability to accurately predict fMRI responses to natural sounds in a new dataset. ( $\mathrm{S}=$ number of sounds; $\mathrm{N}=$ number of features). 
A
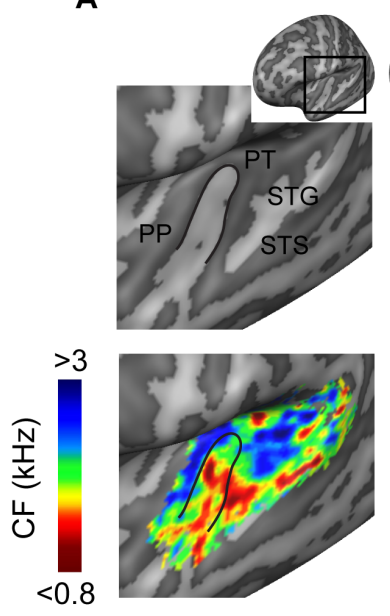

3T
B
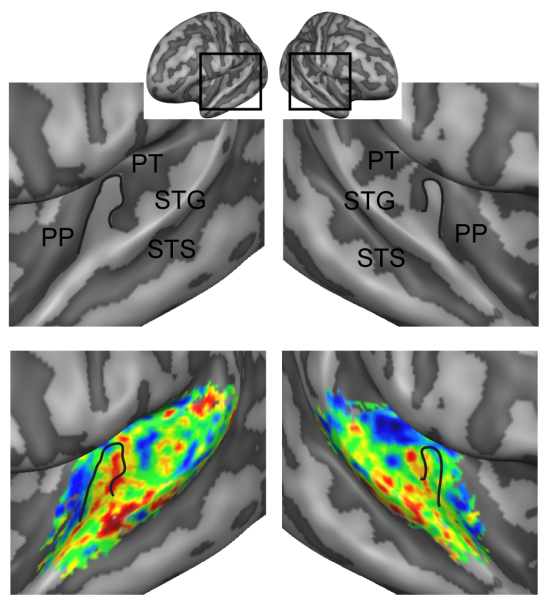

$7 T$

Figure S2.3. Group tonotopic maps. Group maps for the 3T (A) and 7T (B) datasets are displayed on an inflated representation of the group cortex. Maps are shown in the cortical region highlighted by the black square. Group maps are computed as the mean across participants for those voxels that are included in at least 3 individual maps. The black line indicates HG. 
A

Tonotopy (3T)
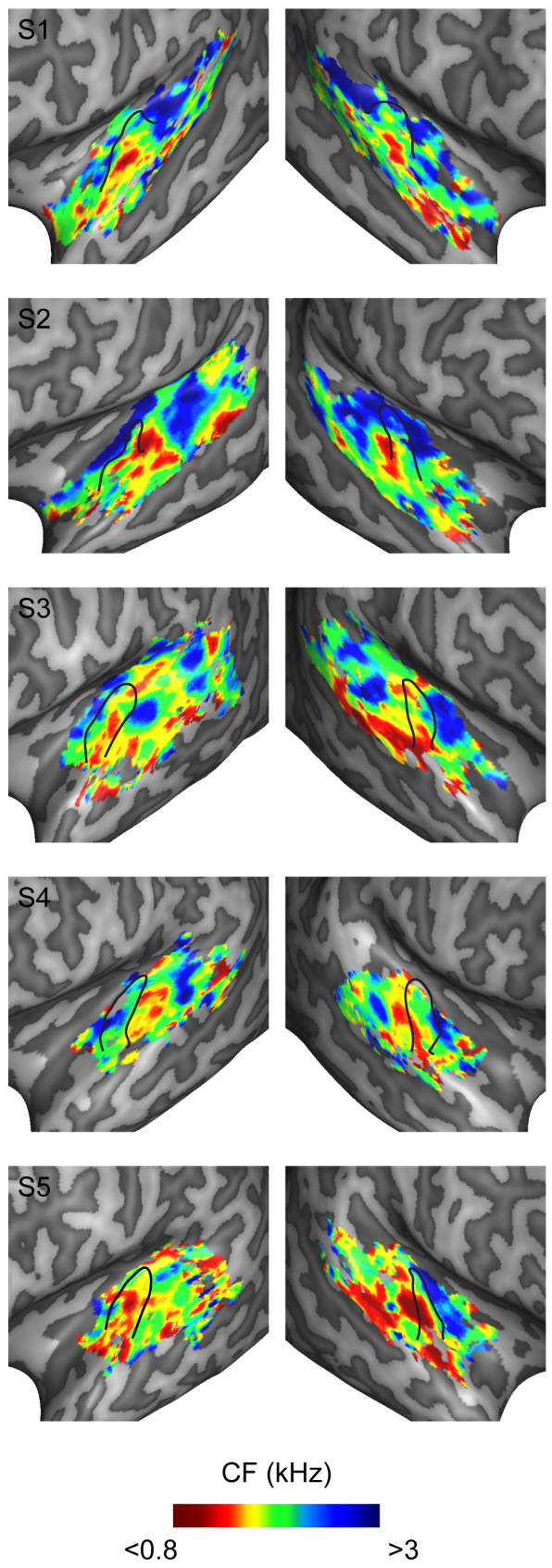

Tonotopy (7T)
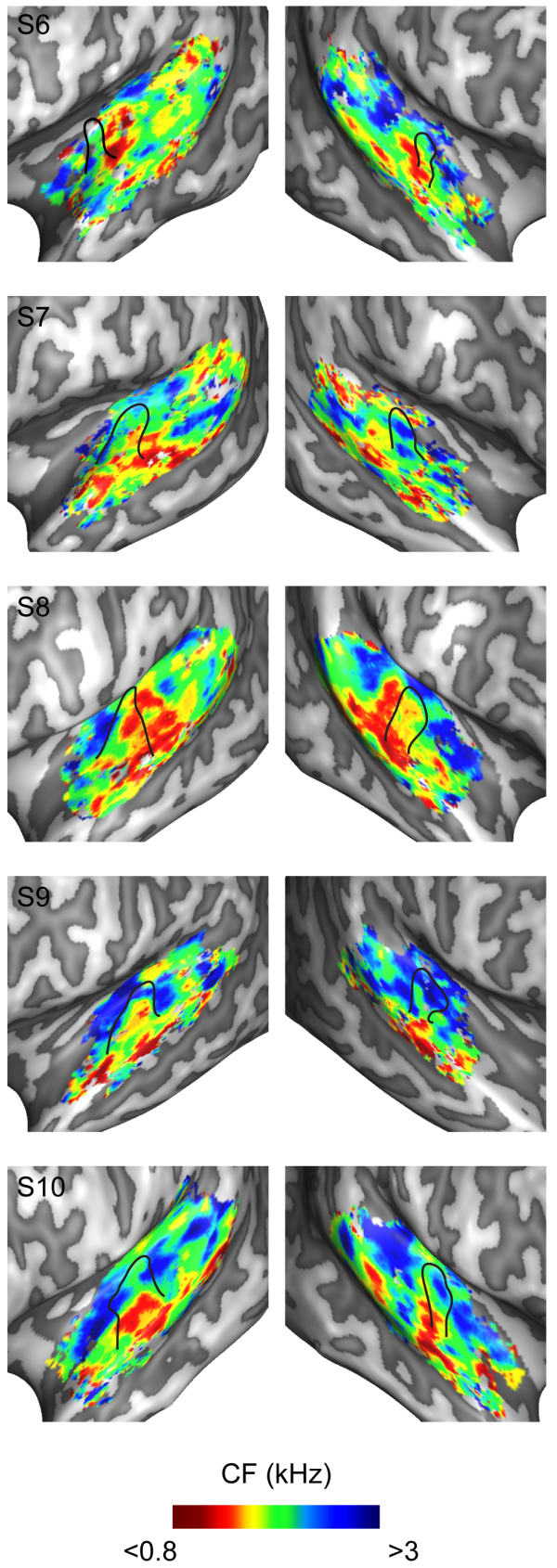

Figure S2.4. Individual tonotopic maps. Individual maps of tonotopy are shown for the $3 T(A)$ and $7 T(B)$ datasets. The black line indicates HG. 
Characteristic

spectral modulation (3T)
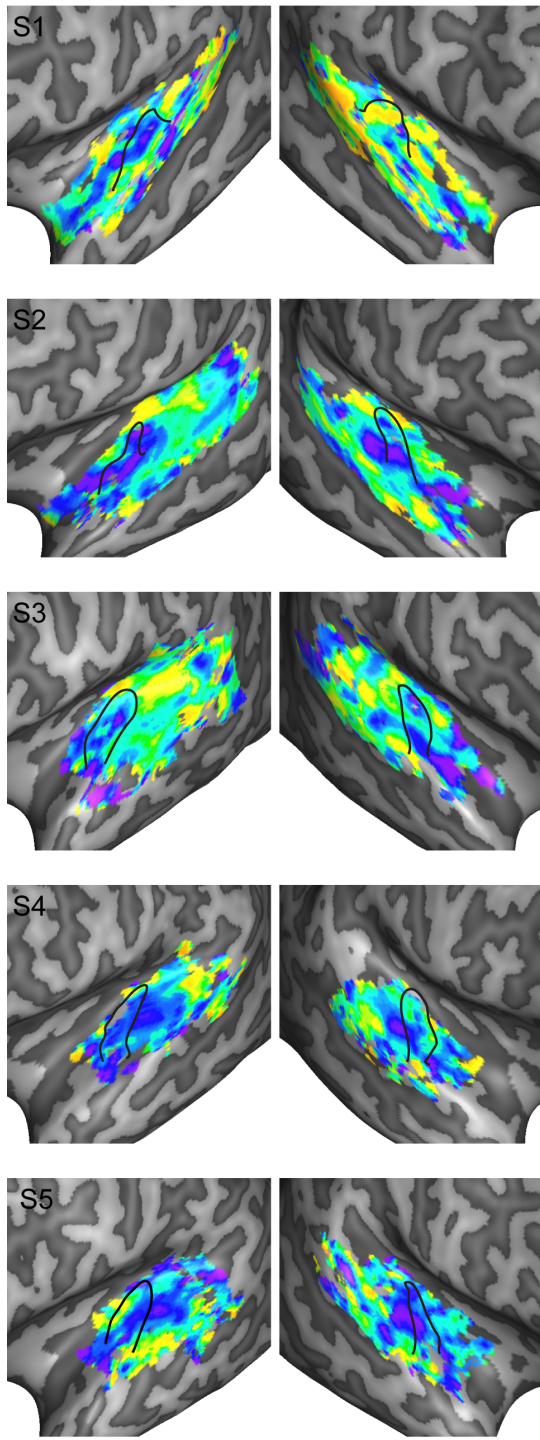

CSM (cyc/oct)

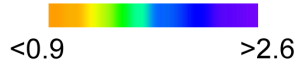

Characteristic temporal modulation (3T)
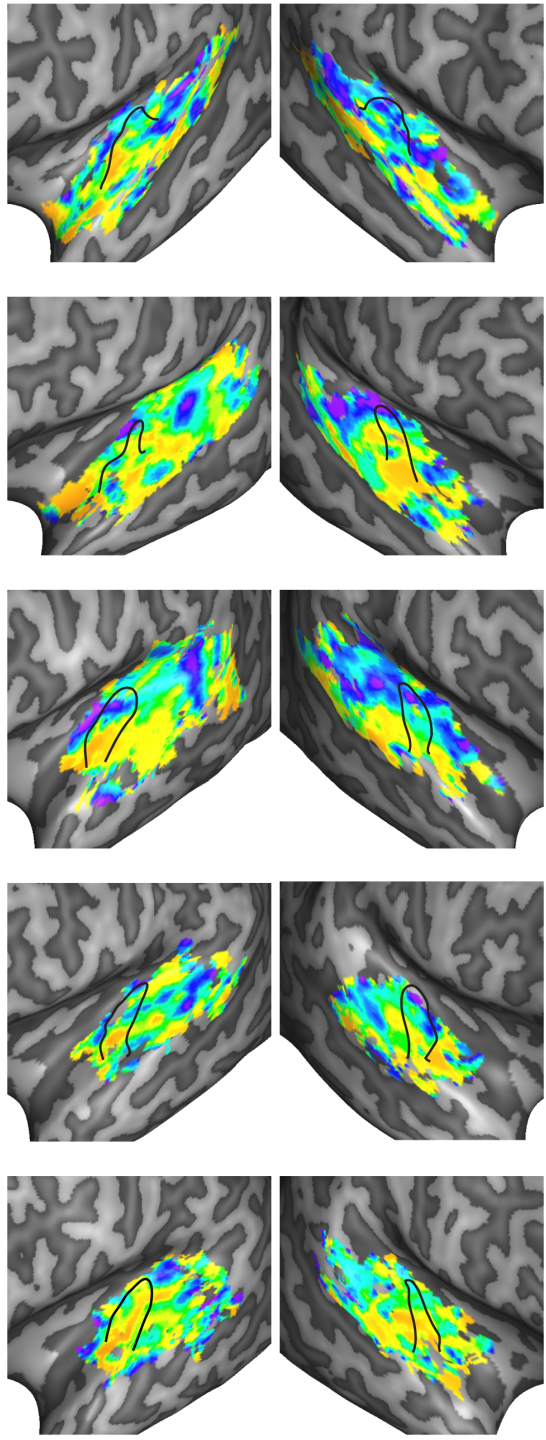

CTM $(\mathrm{Hz})$

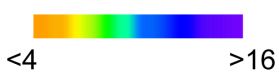

Figure S2.5. Individual topographic maps. Maps of CSM (left) and CTM (right) for all participants in the $3 T$ experiments. Left: purple and orange denote tuning for fine and coarse spectral structures respectively. Right: purple and orange denote tuning for fast and slow temporal variations respectively. The black line indicates HG. 
Characteristic spectral modulation (7T)
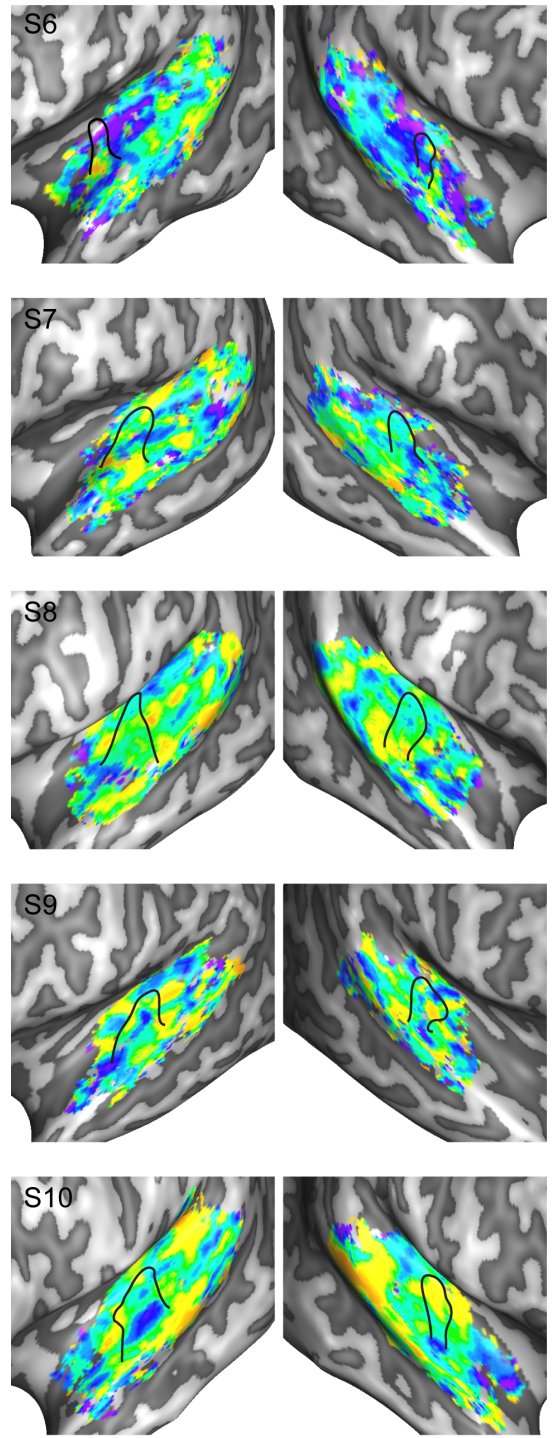

CSM (cyc/oct)

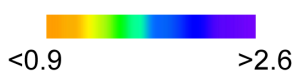

Characteristic temporal modulation (7T)
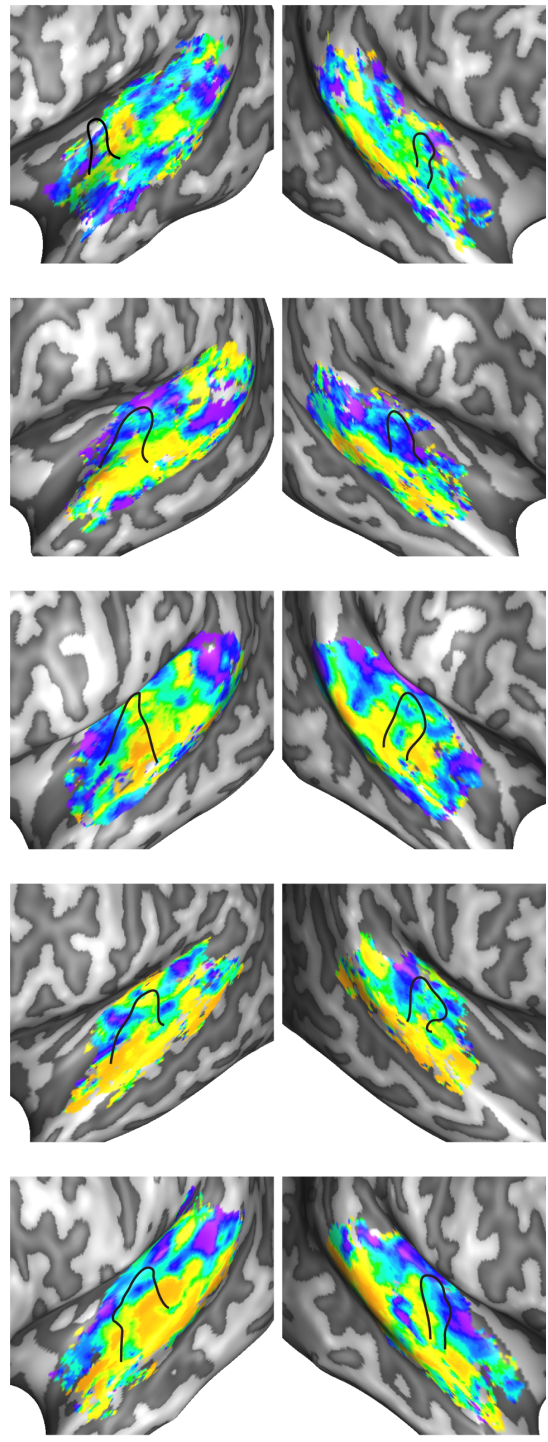

\section{CTM $(\mathrm{Hz})$}

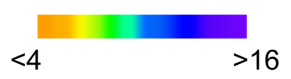

Figure S2.6. Individual topographic maps. Maps of CSM (left) and CTM (right) for all participants in the 7T experiments. Left: purple and orange denote tuning for fine and coarse spectral structures respectively. Right: purple and orange denote tuning for fast and slow temporal variations respectively. The black line indicates HG. 


\section{Supplementary Methods}

\section{Subjects}

Five healthy, normal-hearing subjects (different for the two experiments; median age $=26$ (32), 3 (3) males for the 3T (7T) experiment) gave their informed consent and participated in this study. Additionally to the main study, localizer data were collected in the same subjects for both the $3 \mathrm{~T}$ and $7 \mathrm{~T}$ experiment.

Stimuli for the localizer experiment

In the localizer experiment, we used Matlab (The MathWorks Inc.) to create amplitude modulated tones $(8 \mathrm{~Hz}$, modulation depth of 1$)$ with a carrier frequency of $0.45,0.5$, and $0.55 \mathrm{kHz}$ for the low-frequency condition; $1.35,1.5$, and $1.65 \mathrm{kHz}$ for the middle frequency condition and $2.25,2.5$, and $2.75 \mathrm{kHz}$ for the highfrequency condition. Sounds were sampled at $16 \mathrm{kHz}$ and their duration was cut at $800 \mathrm{~ms}$.

Sound onset and offset were ramped with a $10 \mathrm{~ms}$ linear slope, and their energy (RMS) levels were equalized. Before starting the measurement, sounds were played to the subject while headphones and earplugs were in place. Intensity of the sounds was further adjusted in order to equalize their perceived loudness.

\section{Magnetic Resonance Imaging}

In the $3 T$ experiment, images were acquired on a $3 \mathrm{~T}$ head only MR scanner (Siemens Allegra). Anatomical T1-weighted volumes covering the whole brain were obtained with an ADNI MPRAGE sequence $(T R=2250 \mathrm{~ms}$; $T E=2.6 \mathrm{~ms}$; matrix size $=256 \times 256 \times 192$, voxel dimensions $=1 \times 1 \times 1 \mathrm{~mm} 3$ ). Functional T2*-weighted BOLD images were collected using a clustered volume EPI technique.

Data in the 7T experiment were acquired on a 7T whole body system driven by a Siemens console using a head gradient insert operating at up to $80 \mathrm{mT} / \mathrm{m}$ with a slew rate of $333 \mathrm{~T} / \mathrm{m} / \mathrm{s}$. A head RF coil (single transmit, 16 receive channels) was used to acquire anatomical T1-weighted and functional T2*-weighted BOLD images. T1-weighted images were acquired using a modified MPRAGE sequence $(T R=2500 \mathrm{~ms} ; \mathrm{Tl}=1500 \mathrm{~ms} ; \mathrm{TE}=3.67 \mathrm{~ms}$, voxel dimensions $=1 \times 1 \times 1 \mathrm{~mm} 3)$. Proton density (PD) images were acquired together with the T1-weighted volumes and were used to minimize inhomogeneities in the T1-weighted images (Van de Moortele et al., 2009). Acquisition time for anatomy was around 7 minutes. T2*- 
weighted functional data were acquired using a clustered volume EPI technique.

In the main experiment, $\mathrm{fMRI}$ time series were acquired according to a fast event-related scheme ( $3 \mathrm{~T}$ experiment: $\mathrm{TR}=2600 \mathrm{~ms}$; time of acquisition [TA] = $1200 \mathrm{~ms} ; \mathrm{TE}=30 \mathrm{~ms}$; number of slices $=13$; matrix size $=128 \times 128$; voxel size $=2$ $\times 2 \times 2 \mathrm{~mm} 3$, silent gap $=1400 \mathrm{~ms} ;$ 7T experiment: TR = $2600 \mathrm{~ms}$; TA = $1200 \mathrm{~ms}$; $\mathrm{TE}=30 \mathrm{~ms}$; number of slices $=31$; GRAPPA acceleration X3; partial Fourier 6/8; voxel size $=1.5 \times 1.5 \times 1.5 \mathrm{~mm}^{3}$, silent gap $=1400 \mathrm{~ms}$ ).

The localizer was designed according to a blocked scheme. The acquisition parameters were (3T: TR $=3000 \mathrm{~ms}$; TA $=1500 \mathrm{~ms}$; TE $=30 \mathrm{~ms}$; number of slices = 18; matrix size $=128 \times 128$; voxel size $=2 \times 2 \times 2 \mathrm{~mm} 3$, silent gap $=1500 \mathrm{~ms} ; 7 \mathrm{~T}$ : $\mathrm{TR}=3000 \mathrm{~ms} ; \mathrm{TA}=1500 \mathrm{~ms} ; \mathrm{TE}=30 \mathrm{~ms}$; number of slices = 44; GRAPPA acceleration X3; partial Fourier 6/8; voxel size $=1.5 \times 1.5 \times 1.5 \mathrm{~mm}$, silent gap = $1500 \mathrm{~ms}$ ). Each block ( $18 \mathrm{~s}$ ) consisted of six sounds of the same condition (one sound per TR, presented in the silent gap). Per run, two blocks of each condition were presented (12 s of silence between blocks). Overall, the localizer consisted of six runs ( 9 minutes each).

Analysis of anatomical and functional images was performed with BrainVoyager QX. Preprocessing consisted of slice scan-time correction (sinc interpolation), temporal high-pass filtering and 3-dimensional motion correction. Functional slices were co-registered to the anatomical data and normalized to Talairach space. In the 7T experiment, normalized functional data were resampled (sinc interpolation) to $1 \mathrm{~mm}$ isotropic. The border between gray and white matter was segmented from anatomical volumes and used to generate inflated hemispheres of the individual subjects. Finally, functional cortex based alignment (Goebel et al., 2006) was performed to improve alignment across subjects and compute group maps.

\section{Reliability of parameters estimates: SNR computation}

Maps of voxels tuning preference were derived from the MTFs as estimated by the joint frequency-specific MTF-based model. To assess the reliability of the maps, we computed the signal-to-noise ratio (SNR) of the MTFs estimates, by applying a method developed to evaluate the reproducibility of spectro-temporal receptive fields (STRFs) as derived from neuronal responses (Shechter and Depireux, 2010, 2012). We generated fifty bootstrap samples by drawing with repetition from the original set of responses, and voxels MTFs were estimated for each bootstrap sample. The same bootstrap samples were used for all voxels and 
subjects in order to prevent sample-related variability. The regularization parameter for the fitting procedure was constant across bootstraps and was set to the value derived when the model was trained on the original set of responses. For each voxel, the bootstrap procedure yielded a distribution of parameter estimates at each point $(\omega, \Omega, f)$ of the MTF. The SNR at each $(\omega, \Omega, f)$ was computed as the ratio between the average power and the variance across bootstraps, as follows:

$$
\operatorname{SNR}_{\omega, \Omega, f}=\frac{\left|\mu_{\omega, \Omega, f}\right|^{2}}{\sigma_{\omega, \Omega, f}^{2}}
$$

where $\mu_{\omega, \Omega, f}$ is the mean across bootstraps.

The overall SNR of the MTF was then obtained as the power-weighted mean of all $S N R_{\omega, \Omega, f}$, where the power $P_{\omega, \Omega, f}$ is the square of the MTF computed on the original set of responses (i.e. without bootstrap):

$$
S N R_{M T F}=\frac{\sum_{\omega, \Omega, f} P_{\omega, \Omega, f} S N R_{\omega, \Omega, f}}{\sum_{\omega, \Omega, f} P_{\omega, \Omega, f}}
$$

For every subject, cortical maps of SNR $\mathrm{R}_{\mathrm{MTF}}$ were generated by color-coding the computed SNR values and projecting them onto an inflated representation of the cortex (Figure S2.7). High SNR indicates reproducibility of MTF estimates across bootstraps.

Computation of unbiased topographic maps

To ensure that estimated maps of tuning preference were not confounded by the frequency and modulation content of sound categories, we recomputed maps of CF, CSM and CTM while explicitly accounting for confounding effects of sound category (this analysis was performed on the 7T dataset).

The sounds used in this experiment may be (arbitrarily) grouped into four categories:

1. HA: Human Animate (speech, voice)

2. NA: Nonhuman Animate (animal sounds)

3. HI: Human Inanimate (tools, music) 
A

3T
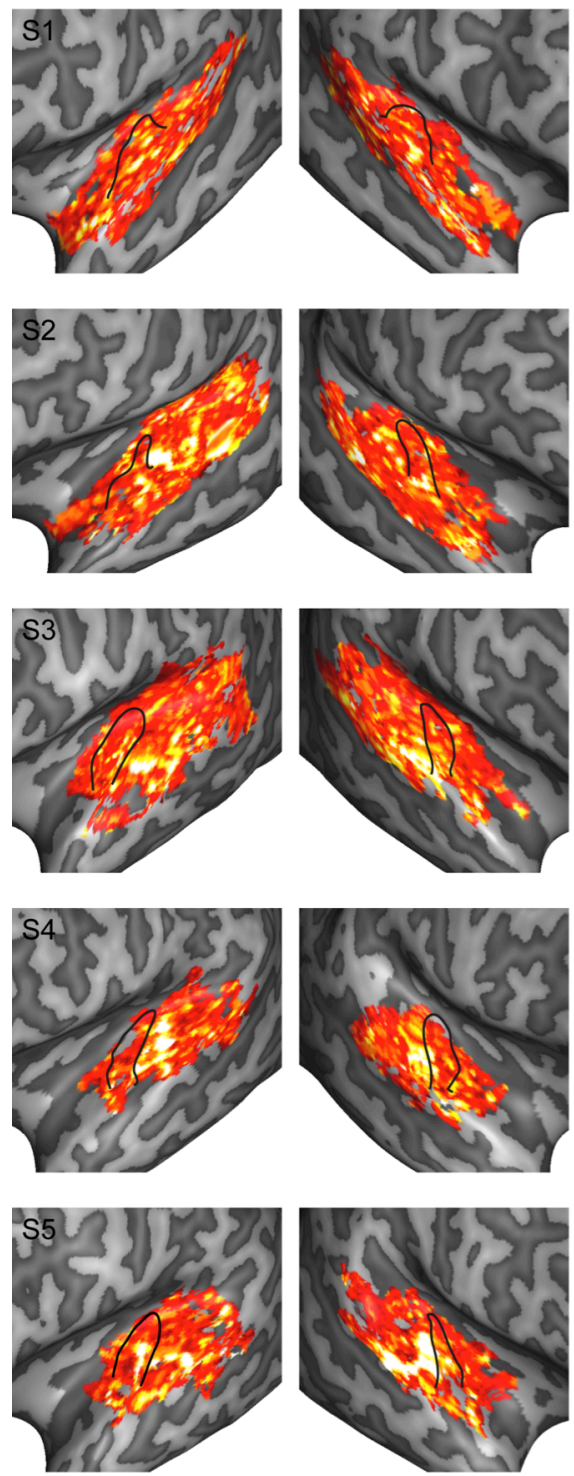

SNR

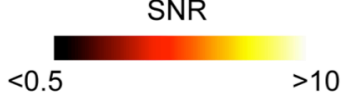

B

$7 \top$
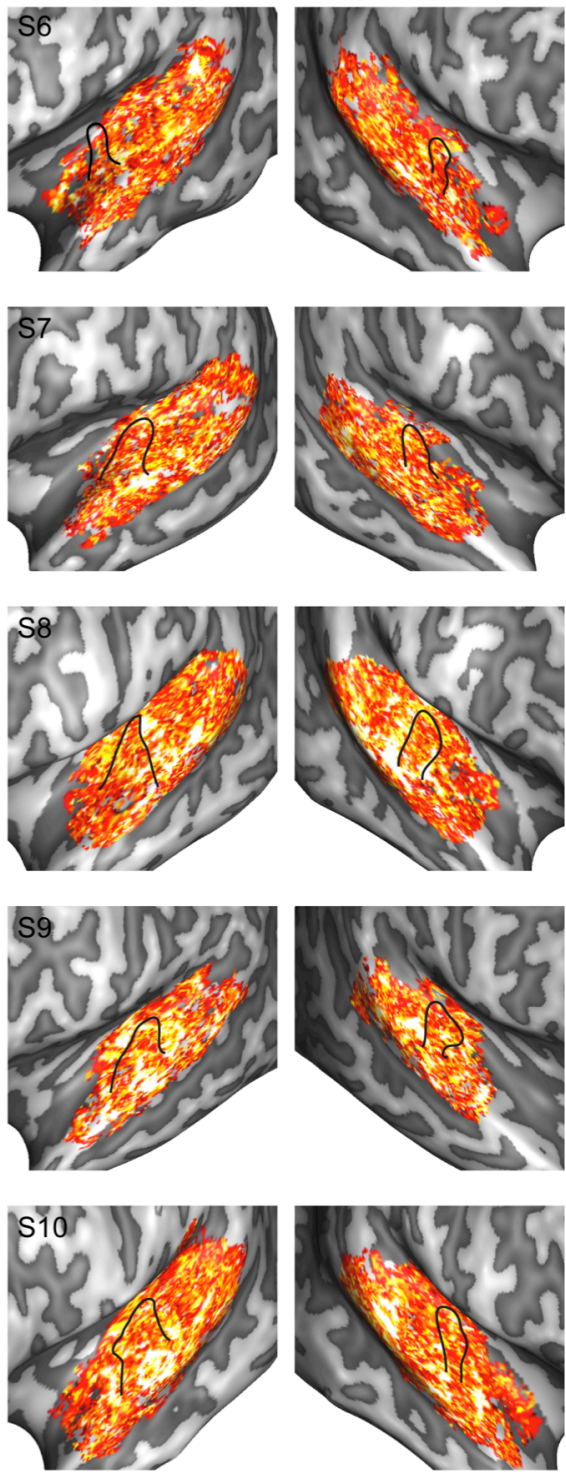

SNR

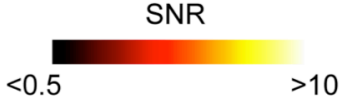

Figure S2.7. Stability of MTFs estimates across bootstraps. Single subjects maps of SNR of voxels MTFs as estimated by the joint frequency-specific MTF-based model at 3T (A) and 7T (B). High values of SNR (bright colors) indicate that the estimated MTF is consistent across bootstraps. The black line outlines HG. 
4. NI: Nonhuman Inanimate (sounds of nature)

For each category, we defined a categorical predictor that takes the value 1 if the stimulus belongs to that category and 0 otherwise. Next, we appended the categorical predictors to the joint frequency-specific MTF-based representation and estimated the parameters of the resulting model. Finally, we derived maps of voxels preferred spectral modulation, temporal modulation and frequency. The spatial patterns of the computed maps remained unchanged (Figure S2.8), indicating that voxels preferred features are estimated correctly despite differences in frequency and modulation content across sound categories.
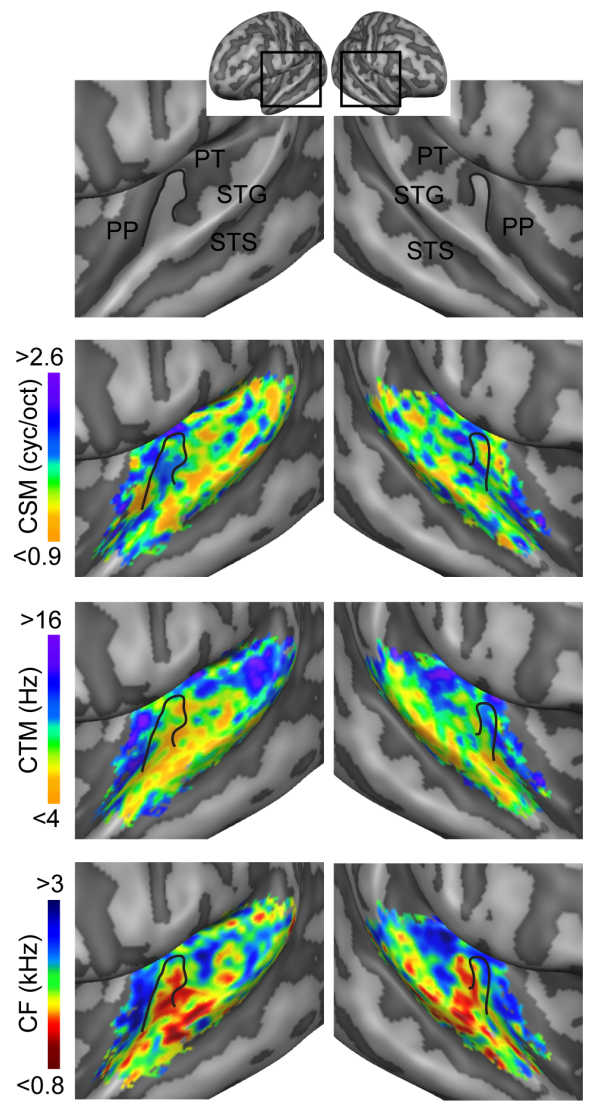

Figure S2.8. Unbiased topographic maps for the 7T dataset. Group maps of CSM, CTM and CF as derived from the joint frequency-specific MTF-based model while explicitly accounting for the effect of sound categories. The black line indicates HG. 


\section{References}

Goebel R, Esposito F, Formisano E (2006) Analysis of functional image analysis contest (FIAC) data with brainvoyager QX: From single-subject to cortically aligned group general linear model analysis and self-organizing group independent component analysis. Hum Brain Mapp 27:392-401

Shechter B, Depireux DA (2010) Nonlinearity of coding in primary auditory cortex of the awake ferret. Neuroscience 165:612-620

Shechter B, Depireux DA (2012) Contrast tuned responses in primary auditory cortex of the awake ferret. Eur J Neurosci 35:550-561

Van de Moortele P-F, Auerbach EJ, Olman C, Yacoub E, Uğurbil K, Moeller S (2009) T1 weighted brain images at 7 Tesla unbiased for Proton Density, T2: contrast and RF coil receive B1 sensitivity with simultaneous vessel visualization. Neuroimage 46:432-446 


\section{Chapter 3}

\section{Spectral and temporal properties of auditory cortical regions and their relation to the acoustic discriminability of natural sounds}

Corresponding manuscript (in preparation):

Santoro R, Moerel M, De Martino F, Valente G, Ugurbil K, Yacoub E, Formisano E. Spectral and temporal properties of auditory cortical regions and their relation to the acoustic discriminability of natural sounds. 


\section{Abstract}

Auditory processing of complex natural sounds engages a network of brain regions throughout the auditory cortex. However, the contribution of these regions to the encoding of natural sounds remains largely unknown. In the present study, we combine high spatial resolution (7 Tesla) functional magnetic resonance imaging ( $\mathrm{fMRI}$ ) with natural sound stimulation and computational modeling to reveal what acoustic components of incoming sounds are encoded within distinct macro-anatomical subdivisions of the human auditory cortex. We apply a modelbased feature reconstruction approach and derive - for each region - its multidimensional tuning profile along three relevant dimensions of natural sounds: frequency, temporal modulations and spectral modulations. Our results show that auditory regions on the superior temporal plane encode a broader range of acoustic features compared to auditory regions along the superior temporal gyrus (STG), where high frequencies and fast temporal modulations are less faithfully represented. Interestingly, in all regions, the temporal modulation profile of the reconstruction accuracy showed a peak at $\sim 3 \mathrm{~Hz}$, a relevant timescale for speech processing and the analysis of syllabic information. Finally, we examine the effect of the observed tuning properties on the acoustic discriminability of incoming sounds and find that already in primary regions - and progressively more in surrounding non-primary regions - the spectral and temporal tuning increases the acoustic distance among speech (and vocal) sounds, but not among exemplars of other categories of natural sounds. These results support the hypothesis that cortical representational mechanisms are optimized for a subset of natural sounds that are behaviorally most relevant. 


\section{Introduction}

Our perception of the auditory world relies on the analysis of incoming sounds by the network of brain regions in the auditory cortex. In numerous neuroimaging studies, the functional role of different auditory cortical regions in the processing of natural sounds has been examined by manipulating stimulus semantic category (e.g. Belin et al., 2000; Lewis et al., 2005; Staeren et al., 2009) or behavioral task (e.g. Ahveninen et al., 2006; Altmann et al., 2007; Formisano et al., 2008; Bonte et al., 2009) (but see Lewis et al., 2009; Leaver and Rauschecker, 2010; Moerel et al., 2012). This experimental approach has provided evidence for regional functional specializations, such as selectivity for conspecific vocalizations along the superior temporal gyrus (Belin et al., 2000) and a dichotomy for sound recognition/localization between anterior-ventral and posterior-dorsal auditory regions (Ahveninen et al., 2006). However, the experimental paradigms used so far do not allow a systematic characterization of which acoustic components of natural sounds are encoded within a certain auditory region. Such knowledge is of paramount importance in order to understand the computations behind high-level functional specializations and to explain why - for example - a given auditory region that shows preferential responses to a certain stimulus category (e.g. voice or speech) also responds vigorously to sounds of other categories (e.g. Staeren et al., 2009).

The acoustic structure of natural sounds consists of complex temporal patterns that combine energy at many frequencies. At the sensory periphery (cochlea), the time varying frequency composition (spectrogram) of incoming sounds is explicitly encoded through an array of frequency-selective channels. In this spectrographic representation, natural sounds exhibit modulations of acoustic energy that span multiple spectral and temporal scales (Singh and Theunissen, 2003). Results from animal electrophysiology (Kowalski et al., 1996; deCharms, 1998), computational modeling (Klein et al., 2003; Chi et al., 2005) and human functional neuroimaging (Santoro et al., 2014) suggest that the cortical processing of natural sounds is mediated by combined neuronal tuning to frequency, spectral and temporal modulations. The present study builds on these previous results and has two aims. A first aim is to detail the contribution of distinct regions of the auditory cortex to the encoding of spectral and temporal sound components. We combine high spatial resolution (7 Tesla) functional magnetic resonance imaging (fMRI) with natural sound stimulation and a model-based feature reconstruction approach (Bialek et al., 1991; Stanley et al., 1999; Miyawaki et al., 2008; Mesgarani et al., 2009; Pasley et al., 2012) to characterize the population tuning of auditory 
cortical regions for frequency, spectral modulations and temporal modulations. In model-based feature reconstruction (Figure 3.1), complex stimuli are decomposed into basic acoustic components (features) using a computational model of auditory processing. Then, multivariate analysis techniques are employed to reconstruct the acoustic components from patterns of brain responses to the stimuli. Finally, the brain-based reconstructions are compared with the original sounds' components. Thus, knowledge about which stimulus components are accurately reconstructed provides a direct measure of what aspects of the incoming sensory information are faithfully encoded by neuronal ensemble responses (Mesgarani et al., 2009). Here, we decompose natural sounds into their frequency-specific spectro-temporal modulation content (see Chapter 2) and use the method of model-based feature reconstruction to characterize the spectral and temporal population response properties of the following anatomical regions of interest (ROIs): Heschl's gyrus $(\mathrm{HG})$, planum polare (PP), planum temporale (PT), anterior, middle and posterior superior temporal gyrus (aSTG, mSTG and pSTG).

A second aim of this study is to assess the relevance of the observed spectral and temporal response properties for the discriminability of natural sounds. Based on electrophysiological recordings in zebra finches, it has been suggested that the spectro-temporal population tuning of auditory neurons is designed to maximize the acoustic distance between sounds, thus facilitating the animal's ability to discriminate between them (Woolley et al., 2005). At present, empirical evidence that a similar mechanism is implemented in the human auditory cortex is lacking. Here, we show that the spectral and temporal population response properties of the HG, PT, aSTG, mSTG and PSTG increase the acoustic distances between speech (and vocal) sounds, but not between exemplars of other categories of natural sounds (e.g. animal cries). Finally, we show that the increase in discriminability between speech sounds is higher in the aSTG, mSTG and PSTG than in the HG and PT.

\section{Results}

ROI-based analysis of spectral and temporal response properties

In this study we pooled together data from two fMRI experiments (for Experiment 1 see De Martino et al., 2013; Moerel et al., 2013; Experiment 2 is based on new measurements, see Material and Methods). In both experiments, $\mathrm{fMRI}$ responses were recorded from the auditory cortex while subjects (Experiment 
A

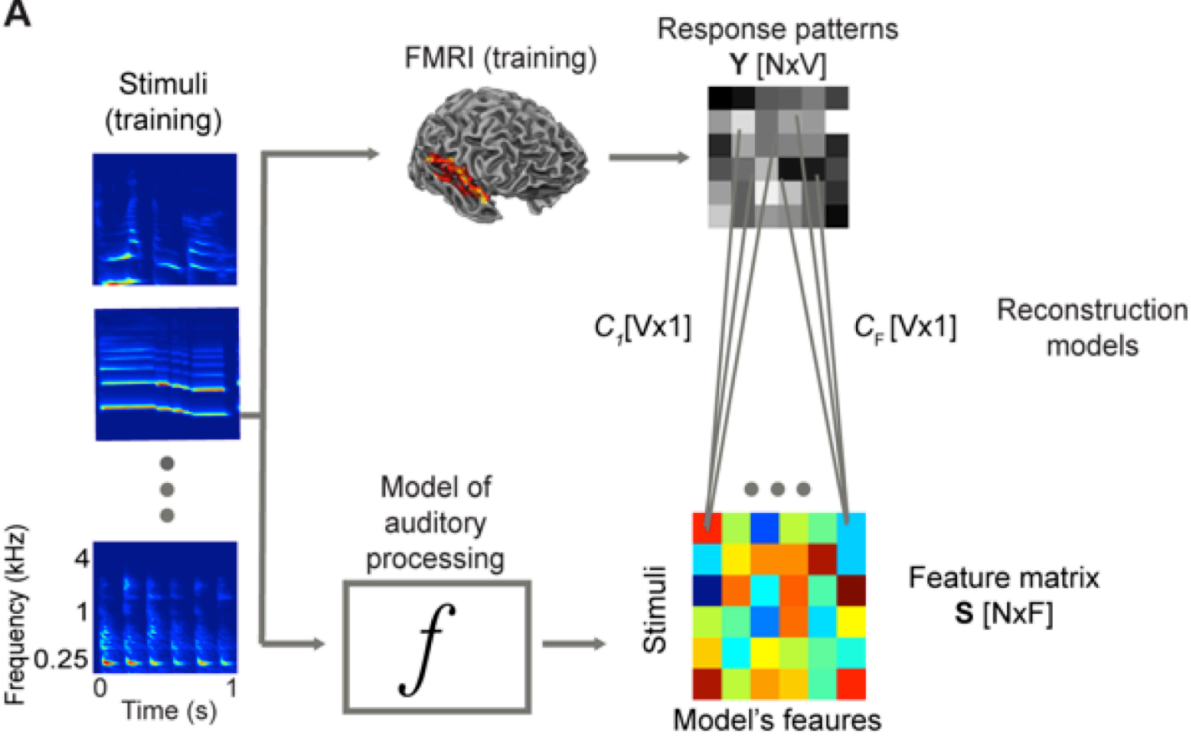

B
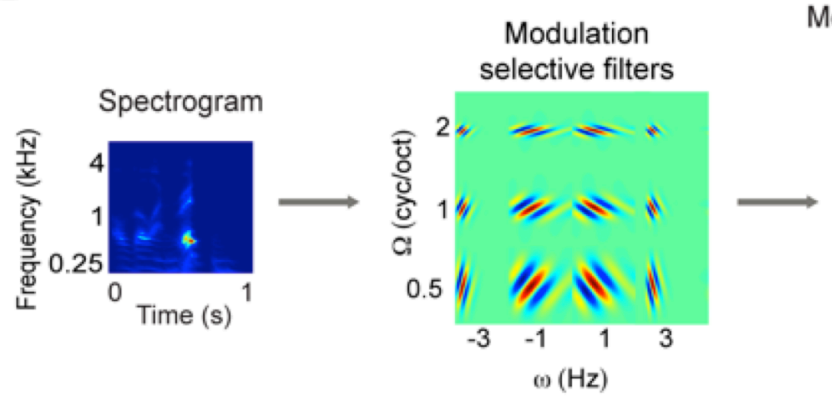

Modulation spectrum

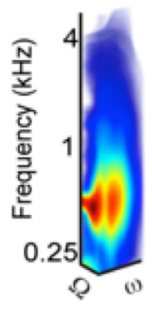

Figure 3.1. Model-based reconstruction procedure and modulation representation. (A) FMRI response patterns to a large set of natural sounds are used to estimate a reconstruction model for each of the acoustic features extracted by a model of auditory processing. To assess reconstruction accuracy, the reconstructed features are compared to the original ones. $\mathrm{N}=$ number of training sounds; $V=$ number of voxels; $F=$ number of acoustic features. (B) The model of auditory processing decomposes the spectrogram through a bank of modulation selective filters tuned to different spectral modulations $(\Omega)$, temporal modulations $(\omega)$, and direction (upwards/downwards). The output of the filter bank is averaged across time and direction to yield a reduced representation of modulation content. This modulation spectrum quantifies the time-averaged amount of energy that is present in the spectrogram at a given combination of $\Omega, \omega$, and frequency (f). Each point $(\Omega, \omega, f)$ of the modulation spectrum constitutes one feature for the reconstruction procedure.

1: $n=5$; Experiment 2: $n=2$ ) listened to a large set of natural sounds, including 
speech samples, vocal sounds, music pieces, animal cries, scenes from nature, and tool sounds (see Materials and Methods).

We investigated the representation of spectral and temporal acoustic features in six anatomical ROls, which were manually labeled on each subject's cortical mesh reconstruction according to the criteria of Kim et al. (2000) (Figure S3.1; see Materials and Methods). ROls from the left and right hemispheres were analyzed separately. Reconstruction models were defined as linear decoders that used patterns of $\mathrm{fMRI}$ activation to predict features of a multi-dimensional stimulus representation (Figure 3.1A). We employed a stimulus representation in which sounds were expressed according to their frequency-localized spectro-temporal modulation content (Chi et al., 2005) (Figure 3.1B; see Materials and Methods).

Using a subset of $\mathrm{fMRI}$ data (training), we estimated a reconstruction model for every feature of the modulation representation. We then assessed the ability of these models to accurately predict sounds' modulation content in a new, independent data set (testing), which involved sounds not used for model estimation. Reconstruction accuracy was quantified as the Pearson's correlation coefficient ( $r$ ) between the reconstructed and the actual stimulus features. This resulted in a modulation transfer function (MTF) per ROI per hemisphere (Figure 3.2). By showing the reconstruction accuracy as a function of frequency, spectral modulation and temporal modulation, the MTF represents a description of which acoustic features can be faithfully reconstructed from the measured population responses. We tested for statistical significance of individual MTFs, as well as pairwise differences between MTFs of different ROIs and inter-hemispheric differences between corresponding ROls (see Materials and Methods). The MTFs of the HG, PP, PT, aSTG and PSTG were averaged across hemispheres, as no significant inter-hemispheric difference was found (exact permutation test, see Materials and Methods).

We observed two main types of MTFs (Figure 3.2 and Figure 3.3). HG, PP and PT exhibited a MTF that spanned the whole range of frequencies and that showed a frequency-dependent spectro-temporal behavior. Specifically, reconstruction accuracy in the spectral modulation dimension $(\Omega)$ was low-pass, with a steeper slope at higher frequencies. In the temporal modulation dimension $(\omega)$, reconstruction accuracy was band-pass with a peak at about $3 \mathrm{~Hz}$ at low and middle frequencies (up to $\sim 2 \mathrm{kHz}$ ), and high-pass at higher frequencies. The frequency profile of the HG and the PT exhibited a U-shaped behavior with lower reconstruction accuracy at about $1 \mathrm{kHz}$. The PP showed a similar profile, although the peak at low frequencies was less pronounced. The MTFs of the aSTG, mSTG 

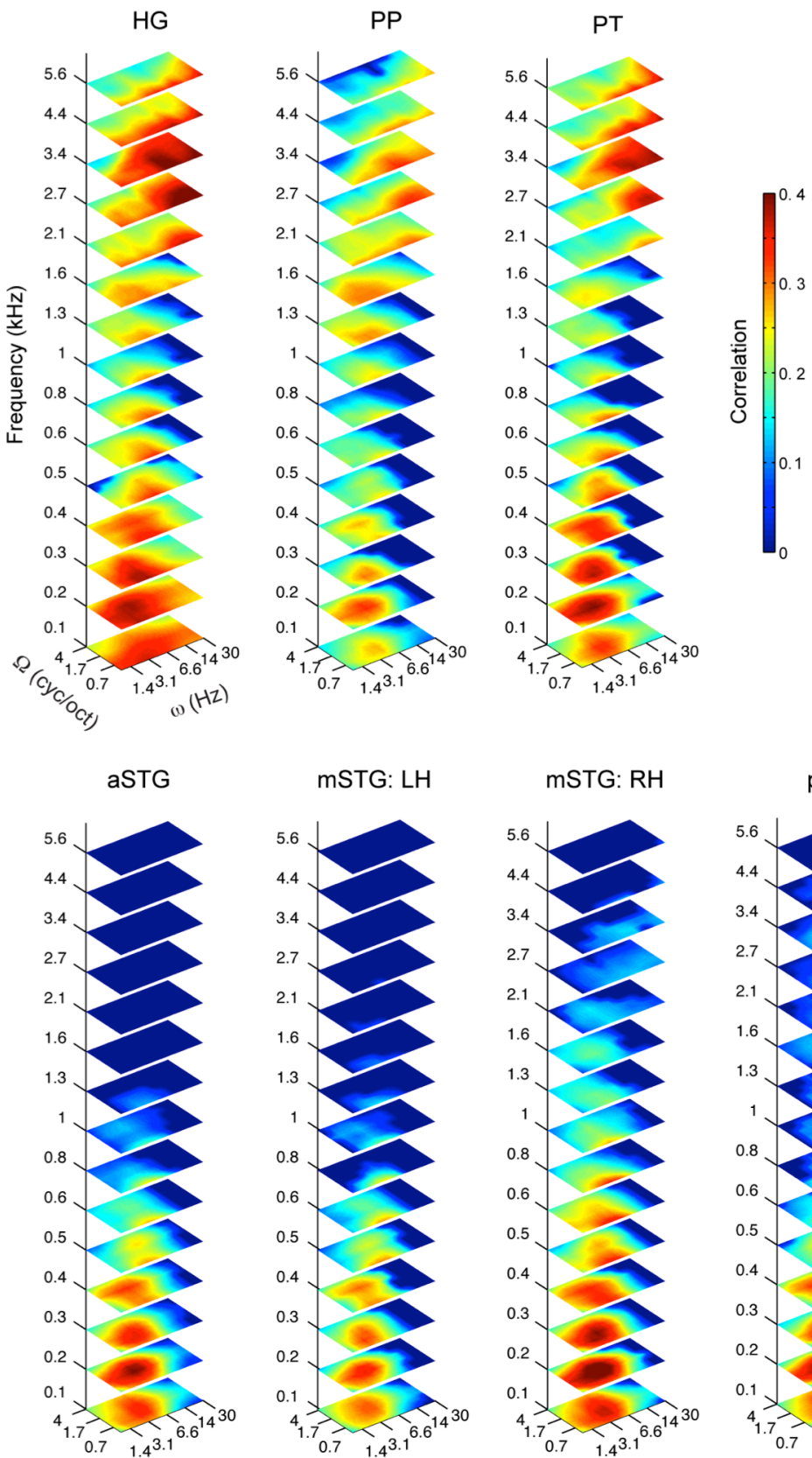

mSTG: LH

mSTG: RH

pSTG
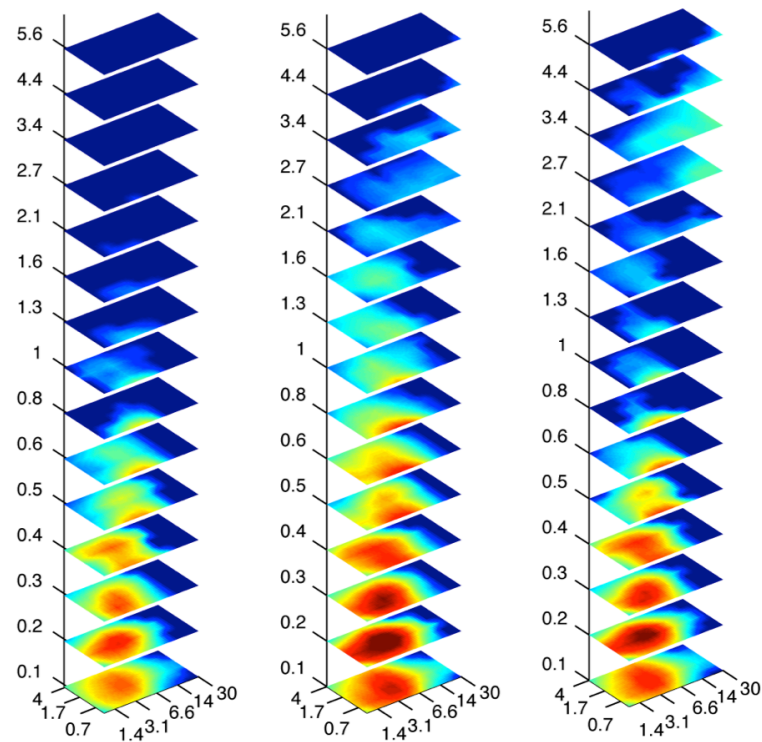

Figure 3.2. MTFs of individual ROls. Each slice represents the MTF at a given frequency value (for simplicity, only 15 frequencies are shown). The color code indicates the group-averaged Pearson's correlation coefficient between reconstructed and original features. Features with a non-significant correlation coefficient (i.e. not accurately reconstructed) are assigned with 0 and depicted ... 
... in dark blue (see Material and Methods for details of the statistical analysis). The average MTF across hemispheres is shown for the HG, PP, PT, aSTG and PSTG. Individual MTFs for the left $(\mathrm{LH})$ and right $(\mathrm{RH})$ hemispheres are shown for the mSTG. MTFs have been interpolated for display purposes. $\Omega$ : spectral modulation frequency; $\omega$ : temporal modulation frequency.

and PSTG were low-pass in frequency, with reconstruction accuracy gradually decreasing at high frequency values. However, the PSTG showed a peak at about 4 $\mathrm{kHz}$. In the temporal modulation dimension $(\omega)$, reconstruction accuracy was bandpass with a peak at about $3 \mathrm{~Hz}$, while it exhibited a low-pass behavior according to spectral modulations $(\Omega)$. These qualitative observations were confirmed by post hoc statistical analyses of the MTFs' marginal profiles (see Material and Methods).

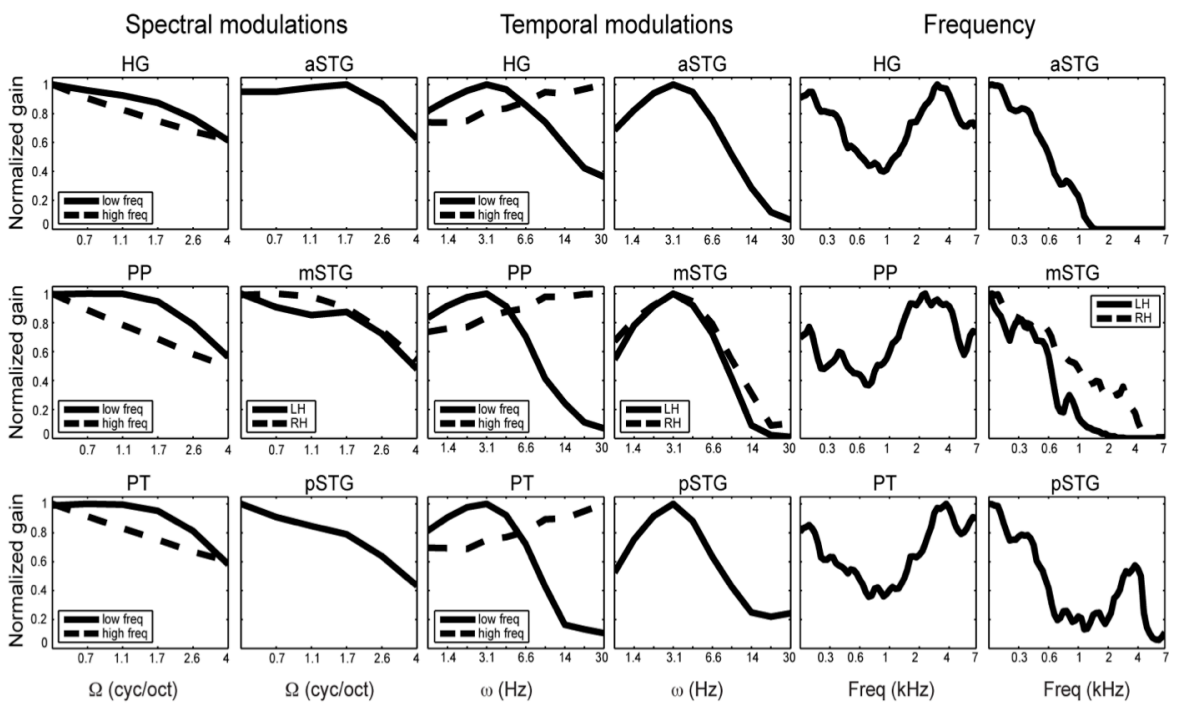

Figure 3.3. Marginal spectral and temporal MTFs. Marginal transfer functions were obtained by averaging the MTFs along irrelevant dimensions (e.g frequency and temporal modulation for the spectral transfer function). For the HG, PP and PT we computed distinct marginal tuning functions for low $(<2 \mathrm{kHz})$ and high frequency values. Separate marginal profiles are shown for the left and right mSTG. Each profile has been normalized by its maximum value. Freq $=$ frequency.

Specifically, for all ROIs, reconstruction accuracy at the lowest spectral modulation (0.5 cyc/oct) was significantly higher than at 4 cyc/oct (Bonferroni adjusted $\mathrm{p}<0.001)$. In the temporal modulation dimension, reconstruction accuracy at $3.1 \mathrm{~Hz}$ was significantly higher than at 1 and $9.7 \mathrm{~Hz}$ for the aSTG, mSTG and pSTG and for 
the HG, PP and PT in the frequency range below $2 \mathrm{kHz}$ (Bonferroni adjusted $\mathrm{p}<0.001)$. At frequencies above $2 \mathrm{kHz}$, reconstruction accuracy of temporal modulations for the HG, PP and PT was higher at $30 \mathrm{~Hz}$ than at $1 \mathrm{~Hz}$ (Bonferroni adjusted $p<0.001$ ). Along the frequency profile, reconstruction accuracy below 0.6 $\mathrm{kHz}$ was significantly greater than at higher frequencies for the aSTG, mSTG and pSTG (Bonferroni adjusted $\mathrm{p}<0.001$ ). For the $\mathrm{HG}$ and the PT, reconstruction accuracy at high $(>2 \mathrm{kHz})$ and low frequencies $(<0.5 \mathrm{kHz})$ was significantly higher than in the mid frequency range (between 0.5 and $2 \mathrm{kHz}$ ) (Bonferroni adjusted $\mathrm{p}<0.001)$. For the PP, the difference between high and mid frequencies was significant (Bonferroni adjusted $p<0.001$ ), but not the one between low and mid frequencies (Bonferroni adjusted $p>0.01$ ).

The mSTG was the only region showing a significant inter-hemispheric difference, with the MTF of the right hemisphere having higher reconstruction accuracies overall (Figure S3.2).

The results of the pairwise comparisons between ROls are reported in Figure 3.4. Compared to the PT, the HG showed significantly greater reconstruction accuracy for high temporal modulations $(>6.6 \omega)$, independently of frequency and spectral modulation. At low frequency values (below $0.5 \mathrm{kHz}$ ), similar differences were observed for the comparisons $H G$ vs aSTG and HG vs pSTG. At higher frequencies $(0.5-1.3 \mathrm{kHz})$, reconstruction accuracy of the $\mathrm{HG}$ was significantly higher than for the aSTG and PSTG for a range of modulations that tended to be band-pass along the temporal dimension and low-pass along the spectral dimension. Finally, reconstruction accuracy of the HG was overall significantly greater than the aSTG and PSTG at frequencies above $1.3 \mathrm{kHz}$. At high frequencies, the HG showed significantly greater reconstruction accuracy than the left and right mSTG. At low frequencies, high temporal modulations were reconstructed with significantly higher accuracy in the HG than in the left mSTG. No significant differences were observed between the $\mathrm{HG}$ and the PP. In the frequency range between 0.5 and $1.3 \mathrm{kHz}$, low temporal modulations $(<6.6 \omega)$ were significantly more accurately reconstructed in the PP than in the aSTG, PSTG and left mSTG. At higher frequencies, reconstruction accuracy of the PP was overall significantly greater compared to the aSTG, pSTG, left and right mSTG. No significant differences were observed between the PP and the PT. At high frequencies ( $>1 \mathrm{kHz}$ ), the PT showed significantly greater reconstruction accuracy than the aSTG, pSTG, left and right mSTG. High frequencies $(>2 \mathrm{kHz}$ ) were significantly less accurately reconstructed in the aSTG compared to the pSTG. In the mid frequency range $(0.5-3.5 \mathrm{kHz})$, reconstruction accuracy of the aSTG was 

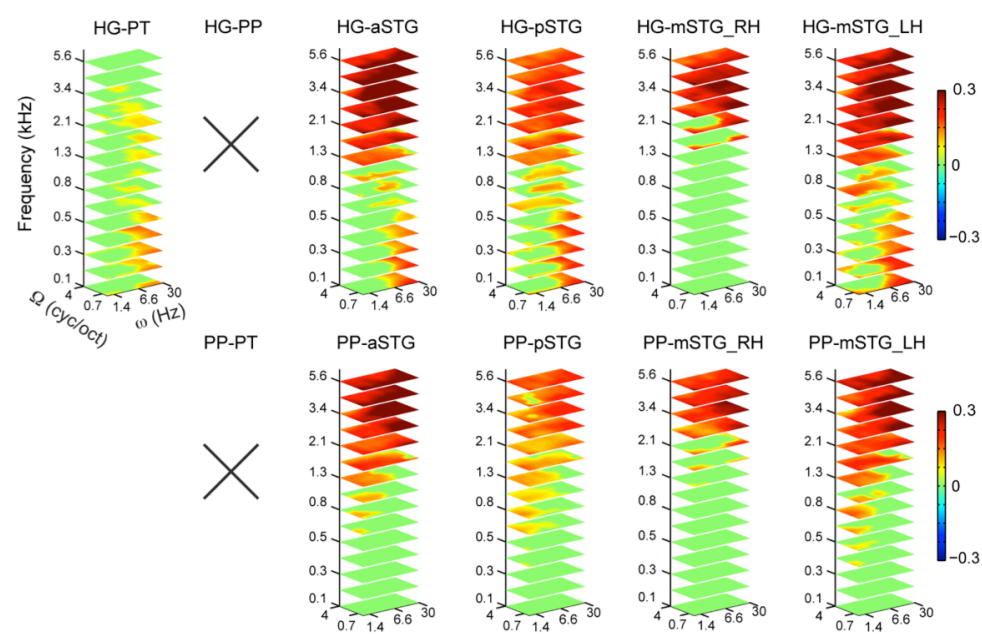

PP-mSTG_LH
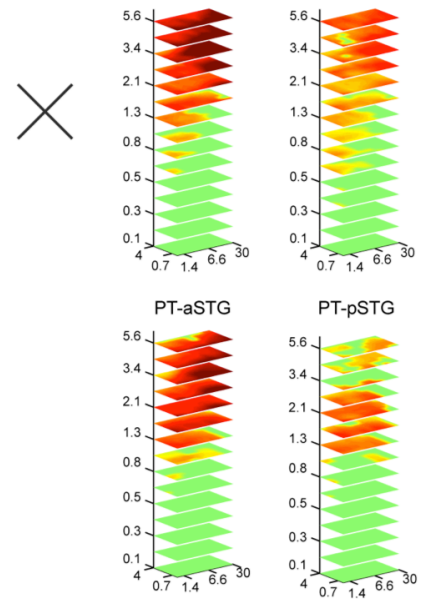

PT-mSTG_RH
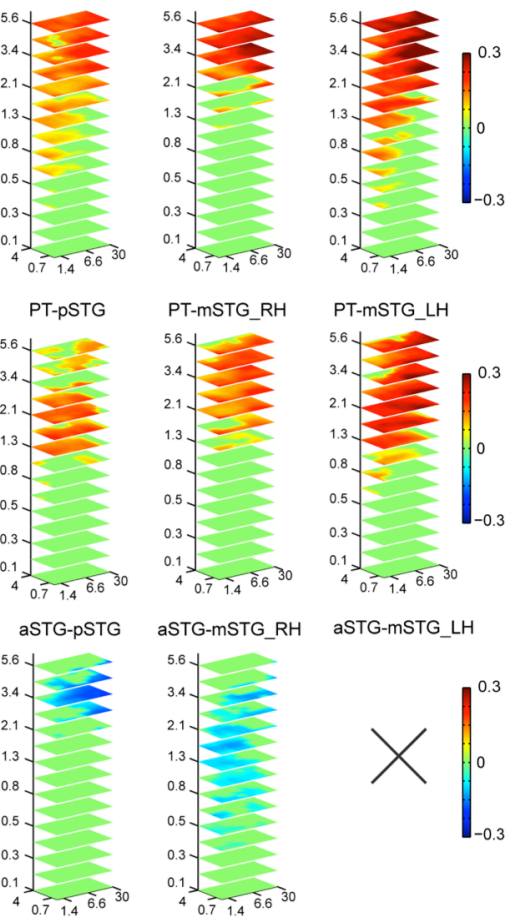

aSTG-mSTG_RH aSTG-mSTG_LH
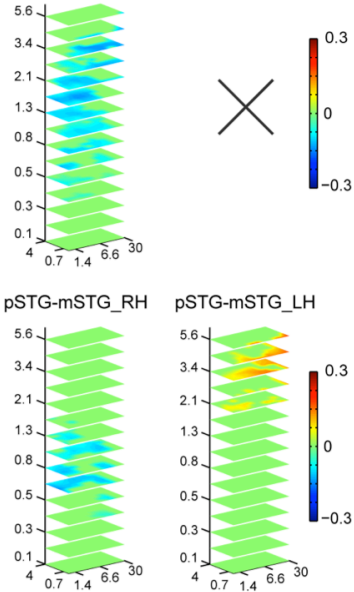

Figure 3.4. Pairwise comparisons between MTFs. In each panel, the color code indicates the group-averaged difference between the MTFs of 2 ROls. Features with a non-significant difference are assigned with 0 and depicted in green (see Material and Methods for details of the statistical analysis). A cross is assigned to pairs of MTFs that are not significantly different. See Figure S3.2 for the difference between the MTFs of right and left mSTG. 
significantly lower than the right mSTG. We did not observe significant differences between the aSTG and the left mSTG. Significant differences between the PSTG and the right $\mathrm{mSTG}$ were localized in the mid frequency range $(0.5-1.3 \mathrm{kHz})$, where the PSTG showed significantly lower accuracies. Conversely, reconstruction accuracy for the PSTG was significantly greater than the left mSTG at high frequencies (> $2 \mathrm{kHz}$ ).

In summary, the ROI-based analysis of spectral and temporal response properties indicates that auditory regions on the superior temporal plane (HG, PP, PT) encode a broader range of acoustic features compared to the STG. Specifically, our data suggest that the aSTG, mSTG and PSTG encode a low-pass representation of the incoming spectrogram with respect to frequency and that fast temporal modulations are less faithfully represented in these regions.

\section{Discriminability analysis}

To test the effect of spectro-temporal response properties on the discriminability of incoming sounds (i.e. on the relative distances between their cortical representation), we filtered the modulation content of our stimuli using the estimated MTFs and compared the pairwise distance between filtered sounds to the distance between pairs of original (unfiltered) sounds. Filtered sounds were obtained as the pointwise product between the original modulation content and the group-average MTF of individual ROls. This can be interpreted as projecting the stimuli into the spectro-temporal representational space of the ROls. We defined the normalized distance between all possible sound pairs as the Euclidean distance between sounds in the modulation space, divided by the maximum pairwise distance obtained for the condition of interest (i.e. filtered or original). When plotted in a 2D graph (distances between filtered sounds vs distances between original sounds), the distribution of normalized pairwise distances provides information on how a given MTF changes the relative distances among sounds. Specifically, points lying on the diagonal designate pairs of sounds whose relative distance does not change after the filtering operation. Conversely, a shift above (below) the diagonal indicates that the filtering operation increases (decreases) the relative distances between sounds, making it easier (more difficult) to discriminate among them. Pairwise distances across the entire stimulus set exhibited a complex pattern (Figure S3.3); however, a regular behavior was observed when the analysis was restricted to distances between pairs of speech sounds (Figure 3.5; Figures S3.4-S3.8 for the other categories). In all ROls, we found a significant linear relationship between the normalized distance of filtered 
HG

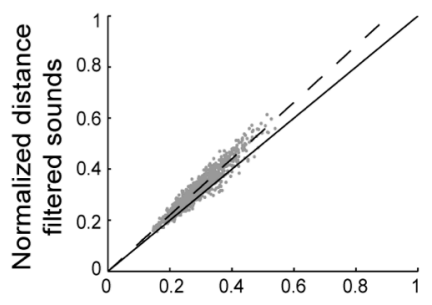

aSTG

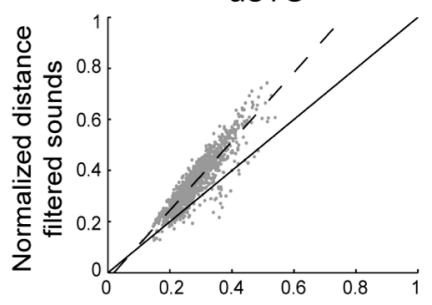

pSTG

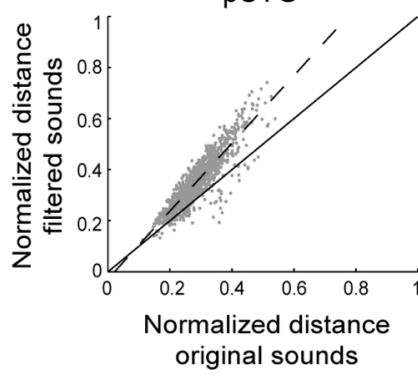

PP

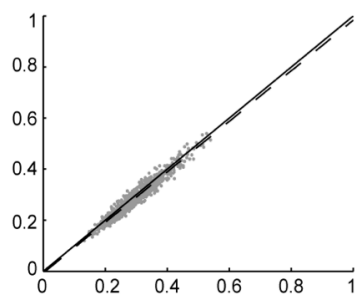

mSTG_RH

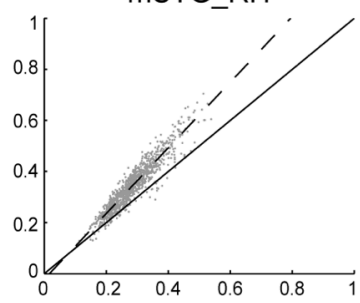

PT

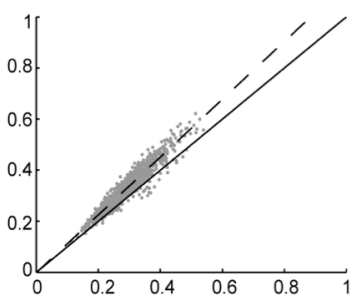

MSTG_LH

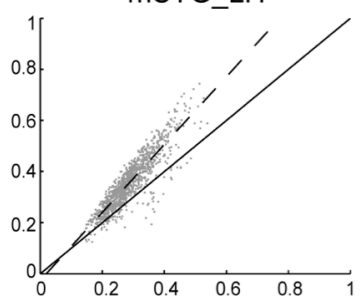

Figure 3.5. Discriminability analysis of speech sounds. Each panel shows the relation between normalized pairwise distances of speech sounds in the original acoustic space and after filtering them with the MTF of one ROI. The dashed line represents the fitted line (see Table 3.1) and the solid line is the diagonal. Points lying on the diagonal designate pairs of speech sounds whose normalized relative distance does not change after the filtering operation. A shift above (below) the diagonal indicates that the filtering operation increases (decreases) the normalized relative distances between speech sounds.

speech sounds and the normalized distance of original speech sounds (Table 3.1). Pairwise distances for the HG, PT, aSTG, mSTG (left and right) and PSTG fell mainly above the diagonal, while they were clustered around the diagonal for the PP. In fact, the slope for the HG, PT, aSTG, mSTG (left and right) and pSTG was significantly higher than 1 (Bonferroni adjusted $p<0.001$; two tailed $z$ test), while in the PP the slope did not significantly deviate from 1 (Bonferroni adjusted $p>0.05$; 
two tailed $z$ test). Additionally, the slope of the regression line was variable across ROIs. Namely, we found that the slope for the HG, PT, aSTG, mSTG (left and right) and PSTG was significantly higher than the slope of PP (Bonferroni adjusted $p<0.001$; two tailed $z$ test; see Table 3.2), and that the regression line was significantly steeper for the aSTG, mSTG (left and right) and PSTG than for the HG and PT (Bonferroni adjusted $\mathrm{p}<0.001$; two tailed $\mathrm{z}$ test; see Table 3.2). These results indicate that the spectro-temporal tuning properties of the PP preserve the original relative distances among speech sounds, while these distances are increased by the MTFs of the other ROls. Additionally, pairwise distances increase more in the aSTG, mSTG and PSTG compared to the HG and PT, suggesting that the spectro-temporal tuning of regions along the STG is especially optimized to favor the discriminability of speech sounds. We did not observe the same behavior for the other sound categories (Figures S3.4-S3.7), except for adults' vocal sounds, whose pairwise distances tended to cluster above the diagonal (Figure S3.8; Table ST3.1). However, we did not observe the same increase in the slope of the regression line when going from regions on the superior temporal plane to regions along the STG (Table ST3.2).

\section{Discussion}

\section{Mechanisms of spectral and temporal processing}

In this study, we used ultra-high field fMRI and a model-based feature reconstruction approach to characterize the spectral and temporal population tuning properties of distinct anatomical ROls, based on voxels' responses to natural sounds. Our results show that the MTFs of auditory regions on the superior temporal plane (HG, PT, PP) span a broader range of acoustic features compared to the MTFs derived along the STG. Specifically, our data indicate that the aSTG, mSTG and PSTG encode a low-pass representation of the incoming spectrogram with respect to frequency. This finding is consistent with previous results showing that auditory regions with a preference for speech/voice sounds and located along the STG exhibit a low-frequency bias (Moerel et al., 2012). Additionally, reconstruction accuracy for high temporal modulation frequencies $(6.6-30 \mathrm{~Hz})$ was highest for the HG (Figure 3.4), indicating a more faithful representation of rapidly varying acoustic information in this region. This result is in line with the HG being the site of the primary auditory cortex (Morosan et al., 2001). 
Table 3.1. Results of linear regression analysis for pairs of speech sounds.

\begin{tabular}{|c|c|c|c|c|c|c|}
\hline & $a$ & $S E_{a}$ & $b$ & $S E_{b}$ & $R^{2}$ & $F(1,1126)$ \\
\hline $\mathrm{HG}$ & $1.10^{\star}$ & 0.01 & -0.001 & 0.003 & 0.92 & $1.33 \times 10^{4 \star}$ \\
\hline PP & $0.99 *$ & 0.01 & $-0.01 *$ & 0.002 & 0.95 & $2.13 \times 10^{4 *}$ \\
\hline PT & $1.13^{\star}$ & 0.01 & -0.002 & 0.003 & 0.91 & $1.19 \times 10^{4 \star}$ \\
\hline aSTG & $1.35^{\star}$ & 0.02 & $-0.03^{\star}$ & 0.01 & 0.81 & $4.91 \times 10^{3 *}$ \\
\hline mSTG_RH & $1.29 *$ & 0.01 & $-0.02^{*}$ & 0.004 & 0.89 & $8.70 \times 10^{3 *}$ \\
\hline mSTG_LH & $1.33^{\star}$ & 0.02 & $-0.03^{\star}$ & 0.01 & 0.80 & $4.57 \times 10^{3 *}$ \\
\hline pSTG & $1.33^{\star}$ & 0.02 & $-0.03^{*}$ & 0.01 & 0.80 & $4.52 \times 10^{3 \star}$ \\
\hline
\end{tabular}

Regression coefficient ( $a=$ slope; $b=$ intercept), standard errors ( $\mathrm{SE}_{a}$ and $\mathrm{SE}_{b}$ ), coefficient of determination $\left(R^{2}\right)$, and corresponding $F$ value. *Bonferroni adjusted $p<0.001$ (two tailed t test for the regression coefficients and $\mathrm{F}$ test for the $R^{2}$ ).

Table 3.2. Pairwise comparisons of linear regression slopes of speech distances.

\begin{tabular}{lcccccc}
\hline & PP & PT & aSTG & mSTG_RH & mSTG_LH & PSTG \\
\hline HG & $9.71^{*}$ & -1.97 & $-11.44^{\star}$ & $-10.82^{\star}$ & $-10.26^{*}$ & $-10.23^{\star}$ \\
PP & 0 & $-11.43^{*}$ & $-17.63^{*}$ & $-19.22^{\star}$ & $-16.27^{\star}$ & $-16.2^{\star}$ \\
PT & 0 & 0 & $-9.98^{*}$ & $-8.91^{\star}$ & $-8.84^{\star}$ & $-8.82^{\star}$ \\
aSTG & 0 & 0 & 0 & 2.73 & 0.79 & 0.78 \\
mSTG_RH & 0 & 0 & 0 & 0 & -1.79 & -1.79 \\
mSTG_LH & 0 & 0 & 0 & 0 & 0 & -0.01 \\
\hline
\end{tabular}

z-scores of pairwise differences between linear regression slopes. Each cell represents the difference between the regression slope of the ROI listed in the row and the regression slope of the ROI listed in the column. *Bonferroni adjusted $p<0.001$.

The frequency profile of the HG, PT, and PP showed a band-stop behavior with a decrease in reconstruction accuracy around $1 \mathrm{kHz}$ (Figure 3.3). This behavior - that was not present in the stimuli (Figure S3.9 and S3.10) - might be related to 
the scanner noise, whose energy was concentrated in this frequency range. In our clustered fMRI acquisition, sounds were presented during silent gaps between scans. It is thus possible that - similar to streaming paradigms (Mesgarani and Chang, 2012) - subjects modulated their attention such that frequencies in the range of the scanner noise were attenuated. Furthermore, the scanner noise between stimuli presentations might have interacted with the response to the auditory stimulation, through e.g. adaptation of the neuronal population of interest or saturation of the BOLD response (Di Salle et al., 2001; Gaab et al., 2007).

The temporal modulation profile of the MTFs exhibited a band-pass behavior that did not match the modulation content of the stimuli (Figure 3.3, Figure S3.9 and Figure S3.10) and showed a peak at $\sim 3 \mathrm{~Hz}$ in all ROls (Figure 3.3). This modulation rate is thought to be one of the relevant timescales for speech processing and it is postulated to be involved in the analysis of syllabic information (Poeppel, 2003). Also, this band-pass profile is in agreement with the temporal modulation transfer function of speech intelligibility (Elliott and Theunissen, 2009) and has been proposed to increase the discriminability between sounds, as opposed to a low-pass temporal modulation profile (Woolley et al., 2005). Indeed, the results of our discriminability analysis support the hypothesis that population tuning for spectral and temporal acoustic features is optimized to facilitate the discrimination between sounds. Interestingly, our data indicate that this mechanism is specific to speech sounds and that occurs as early as the HG, putative location of the primary auditory cortex, although discriminability is higher in regions along the STG. This shows that the spectro-temporal acoustic space encoded by the STG is especially relevant to increase the discriminability of speech sounds. Overall, these results support the hypothesis that neural coding strategies are optimized for a subset of natural sounds that are behaviorally most relevant, rather than for natural sounds per se (Machens et al., 2005). For human listeners, speech is arguably the class of natural sounds with the highest behavioral relevance, as it mediates communication. In our everyday life, discriminating between speech sounds is more crucial than distinguishing e.g. between two dogs barking. Therefore, it is reasonable that the brain has developed mechanisms to selectively amplify the acoustic distances between speech sounds. It remains to be clarified how this mechanism is shaped by experience.

The effects of spectral ad temporal response properties on sounds' discriminability were assessed by multiplying point-by-point the modulation content of our stimuli with the estimated MTFs. Note that our MTFs represent reconstruction accuracies rather than amplitude gains and thus they are different 
from traditional functions that relate stimulus features to the intensity of evoked neuronal/BOLD responses. The interpretation of our MTFs as filters assumes that estimated reconstruction accuracies reflect the amount of information about stimulus features that is encoded within a given ROI. Multiplication of a regionspecific MTF with the stimuli, thus, mimics the "filtering" of acoustic information content in that region.

It has been proposed that a spectro-temporal trade off exists between the hemispheres, with the left auditory cortex specialized for processing rapid temporal changes and a complementary preference in the right auditory cortex for finegrained spectral analysis (Zatorre and Belin, 2001). We found a statistically significant difference only between the MTFs of the right and left mSTG. This difference appeared to be broadly distributed across the acoustic space spanned by the mSTG; however, the higher reconstruction accuracy for high spectral modulation frequencies that was observed in the right mSTG is in favor of the proposed rightward bias for fine-grained spectral processing (Figure S3.2).

Advantages and limitations of the proposed methodological procedure

Our approach differs from previous investigations of cortical spectro-temporal acoustic processing in terms of both stimulus type and data analysis strategy. Previous neuroimaging studies have investigated the cortical representation of frequency, spectral modulations and temporal modulations by measuring brain responses to synthetic stimuli that varied parametrically in the dimension of interest - e.g. tones for frequency (Formisano et al., 2003; Langers et al., 2007; Da Costa et al., 2011), artificial stimuli with different degrees of spectral complexity for spectral modulations (Schönwiesner et al., 2005; Warrier et al., 2009), amplitude modulated noise for temporal modulations (Giraud et al., 2000; Barton and Venezia, 2012; Herdener et al., 2013), dynamic ripples for spectro-temporal modulations (Langers et al., 2003; Schönwiesner and Zatorre, 2009). Despite synthetic sounds allow a higher control over the acoustic properties of the stimuli, they lack both the behavioral relevance and statistical structure of natural sounds. It has been shown that tuning properties of auditory neurons differ significantly under natural and synthetic stimulus condition (Theunissen et al., 2000; Bitterman et al., 2008; Laudanski et al., 2012); therefore it is not clear how results derived using synthetic stimuli generalize to the processing of natural sounds (see Chapter 2). A second limitation of previous neuroimaging studies is that frequency, spectral and temporal modulations have been considered in isolation, i.e. independent processing mechanisms have been assumed for these acoustic dimensions. 
However, we have shown that a model that describes voxels as filters with combined tuning to frequency, spectral modulations and temporal modulations is a better representation of $\mathrm{FMRI}$ responses to natural sounds, compared to models that postulate independent tuning for these dimensions (Santoro et al., 2014). This suggests that concurrent stimulus manipulations in all three dimensions are necessary in order to reliably characterize the tuning properties of auditory cortical regions.

Few fMRI studies have investigated the spectral and temporal properties of the auditory cortex using natural sounds (Moerel et al., 2012, 2013; Santoro et al., 2014). The only study that explored the combined processing of frequency, spectral modulations and temporal modulations addressed the question of the spectro-temporal specialization of distinct auditory cortical areas by looking at the spatial distribution of voxels' tuning preferences throughout the auditory cortex (Santoro et al., 2014). For each voxel, Santoro et al. (2014) estimated a 3D MTF that described voxel's tuning along the dimensions of frequency, spectral and temporal modulation. Then, the authors computed cortical maps of preferred frequency, spectral and temporal modulation by averaging each MTF across the irrelevant dimensions and assigning each voxel with the peak of the resulting 1dimesional marginal tuning curves. Although this approach is useful to reveal regional variations of frequency and modulation tuning properties, it might not be optimal to characterize the population response of voxels within distinct auditory regions. First, this method is based on univariate models of individual voxel responses and does not take into account spatial patterns of fMRI responses, which have been shown to carry important information for the decoding of a stimulus (Haxby et al., 2001). Second, by assigning voxels with the peak of their tuning curve, this approach provides only partial information about voxels with complex response profiles (Moerel et al., 2013). Third, it is not straightforward to infer how sounds are encoded across a population of voxels on the basis of tuning properties derived from the univariate modeling of individual voxels (see Mesgarani et al., 2009, and Chapter 5). The model-based stimulus reconstruction approach that we implemented in the present study allowed overcoming these limitations. By exploiting patterns of brain activity to reconstruct basic components of natural sounds, we were able to directly characterize the spectral and temporal response properties of populations of voxels within distinct regions of the auditory cortex. Nevertheless, one important caveat should be mentioned. We restricted our analysis to ROls that were defined on the basis of macro-anatomical landmarks. However, cytoarchitectonic studies indicate that macro-anatomical regions embed 
several auditory fields (Morosan et al., 2001, 2005; Wallace et al., 2002), which are possibly functionally different. This limitation stems from the current absence of methods to reliably outline auditory cortical fields in individual subjects in vivo. However, our methodological procedure can readily be applied to the functional characterization of auditory cortical fields when techniques for in vivo MRI based brain histology will be established (Geyer et al., 2011; Deistung et al., 2013).

\section{Conclusions and perspectives}

The present study demonstrates how fMRI and model-based feature reconstruction techniques can be successfully combined to study the representation of natural sounds in distinct regions of the human auditory cortex. By proving the possibility of accurately reconstructing individual acoustic components of natural sounds from fMRI response patterns, we pave the way towards fMRI-based re-synthesis of natural sounds (see Chapter 4). Finally, our findings on the mechanisms that the auditory cortex implements to enhance acoustic distances between speech sounds might have relevant implications for the design of systems for automatic speech recognition, whose performance rely heavily on the representation of the input signal (Kleinschmidt, 2002).

\section{Materials and Methods}

\section{Experimental procedure}

Subjects, stimuli, experimental design, MRI parameters, and data preprocessing relative to Experiment 1 have been described in detail in Chapter 2 . Briefly, fMRI responses were recorded from the auditory cortex while subjects ( $n=$ 5) listened to a set of 168 recordings of natural sounds (duration: 1 s, sampling frequency: $16 \mathrm{kHz}$ ). Stimuli included human vocal sounds (both speech and nonspeech, e.g., baby cry, laughter, coughing), animal cries (e.g., dog, cat, horse), musical instruments (e.g., piano, flute, drums), scenes from nature (e.g., rain, wind, thunder), and tool sounds (e.g., keys, scissors, vacuum cleaner). Data were subdivided into six training runs and two testing runs. In the training runs, 144 of the 168 stimuli were presented with 3 repetitions overall (i.e. each sound was presented in 3 of the 6 training runs). The remaining 24 sounds were presented in the testing runs and repeated 3 times per run. Only data from the training runs were analyzed in this study. FMRI time series were acquired according to a fast event-related scheme $(T R=2600 \mathrm{~ms}$; TA $=1200 \mathrm{~ms}$; TE $=30 \mathrm{~ms}$; number of slices 
= 31; GRAPPA acceleration X3; partial Fourier 6/8; voxel size $=1.5 \times 1.5 \times 1.5$ $\mathrm{mm} 3$, silent gap $=1400 \mathrm{~ms})$. Within each run, stimuli were randomly spaced at a jittered inter-stimulus interval of 2, 3, or 4 TRs and presented, with additional random jitter, in the silent gap between acquisitions.

The experimental procedure relative to Experiment 2 will be described in detail in the following.

\section{Subjects}

Two healthy subjects (mean age $=28$ years, 1 male) participated in Experiment 2. Subjects reported no history of hearing disorder or neurological disease, and gave informed consent before commencement of the measurements. The Ethical Committee of the Faculty of Psychology and Neuroscience at Maastricht University granted approval for the study.

\section{Stimuli}

Stimuli for Experiment 2 consisted of 288 recordings of natural sounds. Stimuli were equally distributed among six semantic categories (48 sounds per category): human non-speech vocal sounds, speech sounds, animal cries, musical instruments, scenes from nature, and tool sounds. Vocal sounds were equally distributed among male, female and children's voices. Speech sounds were evenly divided between male and female speakers. Sounds were sampled at $16 \mathrm{kHz}$ and their duration was cut at 1000 ms. Sound onset and offset were ramped with a 10 ms linear slope, and their energy (RMS) levels were equalized. Sounds were delivered binaurally through fMRI-compatible earphones (Sensimetrics Corporation).

\section{MRI parameters}

MRI data for Experiment 2 were acquired on the 7T system at Scannexus (www.scannexus.nl, Maastricht, The Netherlands). A head RF coil (single transmit, 32 receive channels) was used to acquire anatomical (T1-weighted) and functional (T2*-weighted BOLD) images. Anatomical T1-weighted images were acquired using a Magnetization Prepared Rapid Acquisition Gradient Echo (MPRAGE) sequence $(\mathrm{TR}=3100 \mathrm{~ms} ; \mathrm{TI}=1500 \mathrm{~ms}$; flip angle $=5$ degrees; voxel size $=0.6 \mathrm{x}$ $0.6 \times 0.6 \mathrm{~mm}^{3}$ ). Proton density weighted (PD-weighted) images were also acquired $\left(\mathrm{TR}=1440 \mathrm{~ms}\right.$; voxel size $\left.=0.6 \times 0.6 \times 0.6 \mathrm{~mm}^{3}\right)$. The PD-weighted images were used to minimize inhomogeneities in T1-weighted images (Van de Moortele et al., 
2009). Acquisition time for anatomy was $\sim 10 \mathrm{~min}$. T2*-weighted functional data were acquired using a clustered echo planar imaging (EPI) sequence in which time gaps were placed after the acquisition of each volume. FMRI time series were acquired according to a fast event-related scheme, with the following acquisition parameters: TR $=2600 \mathrm{~ms}$; TA $=1200 \mathrm{~ms}$; TE $=19 \mathrm{~ms} ;$ GRAPPA $=2$; partial Fourier $=6 / 8$; flip angle $=70$ degrees; voxel size $=1.5 \times 1.5 \times 1.5 \mathrm{~mm}^{3}$. Between subsequent acquisitions there was a silent gap of $1.4 \mathrm{~s}$ during which the sounds were presented. Slices covered the brain transversally from the inferior portion of the anterior temporal pole to the superior portion of the superior temporal gyrus bilaterally.

\section{Data collection}

Stimuli were divided into 4 non-overlapping sets of 72 sounds each. Grouping was performed randomly under the constraint that all semantic categories (see above) would be equally represented in each set. Each subject underwent two scan sessions. During one session, 2 of the 4 sets of stimuli were presented. The order of the stimulus sets was counterbalanced across subjects. Each session consisted of six functional runs ( 11 minutes each). We presented one stimulus set (72 distinct sounds) per run and every set was presented three times (i.e. 3 runs per set). Within each run, stimuli were arranged according to a pseudo-random scheme to ensure that all semantic categories would be uniformly distributed throughout the run and that no stimuli of the same category would follow each other. Within each scan session, the stimulus sets were presented in an interleaved fashion.

Within each run, stimuli were randomly spaced at a jittered inter-stimulus interval of 2, 3, or 4 TRs and presented, with additional random jitter, in the silent gap between acquisitions. Five "zero" trials (trials where no sound was presented) and five "catch" trials (trials in which the preceding sound was repeated) were included. Subjects were instructed to respond with a button press when a sound was repeated. This incidental 1-back task ensured that participants remained alert and focused on the stimuli. Catch trials were excluded from the analysis.

\section{Data pre-processing}

Functional and anatomical data were pre-processed with BrainVoyager QX (Brain Innovations, Maastricht, The Netherlands). Preprocessing consisted of temporal high-pass filtering (removing drifts of seven cycles or less per run), and 
3D motion correction (trilinear/sinc interpolation). No spatial smoothing was applied. Anatomical data from the two scan sessions were aligned using the automatic alignment tools available in BrainVoyager QX (Brain Innovations, Maastricht, The Netherlands). Functional slices were co-registered to the anatomical data and normalized in Talairach space. Normalized functional data were resampled (sinc interpolation) to $1 \mathrm{~mm}$ isotropic resolution. The border between gray and white matter was segmented from anatomical volumes and used to generate cortical surface meshes of the individual subjects. We performed cortex-based alignment (Goebel et al., 2006) of all the seven subjects included in this study. Alignment information was used to obtain a group surface mesh representation and to compute the overlap of ROls across subjects (see Figure S3.1).

\section{Definition of Anatomical ROls}

We parcellated the superior temporal cortex into the following anatomicallydefined ROls: Heschl's gyrus (HG), planum polare (PP), planum temporale (PT), anterior, middle and posterior superior temporal gyrus (aSTG, mSTG and pSTG). ROls were manually labeled according to the criteria defined in (Kim et al., 2001). The HG is the most anterior transverse gyrus on the superior temporal plane. Its antero-medial border was defined by the first transvers sulcus (FTS) and its posterolateral border by the Heschl's sulcus (HS), or by the sulcus intermedius (SI) in case it was present. Medially, the HG was confined by the circular sulcus of the insula (CSI).

The PP is a region on the superior temporal plane anterior to HG. Medially, the PP was confined by the CSI. The lateral border was defined following the FTS until the anterior end of the HS or the SI; here, the lateral border was changed to the lateral rim of the superior temporal plane.

The PT is a triangular region posterior to $\mathrm{HG}$ on the superior temporal plane. Its antero-medial border was defined by the HS or by the SI when it was present. The lateral rim of the superior temporal plane confined the PT laterally; the medial border was defined as the deepest point of the Sylvian fissure from the medial origin of HS until the posterior point of the STG at the temporal parietal junction.

The superior temporal gyrus constitutes the lower bank of the Sylvian fissure and runs parallel to the superior temporal sulcus (STS). It was traced from its anterior border at the temporal pole to the posterior end of the Sylvian fissure at the temporal parietal junction. The STG was additionally subdivided into anterior, 
middle and posterior portions (aSTG, mSTG and pSTG). The anterolateral and posteromedial ends of the HG were used as reference points to trace the borders of the mSTG.

Anatomical ROls were manually outlined on each subject's cortical surface meshes using BrainVoyager OX (Brain Innovation). We obtained 3D ROls by projecting the selected regions into the volume space of the same subjects. To ensure that no overlap between distinct ROls was present, all 3D ROls were visually inspected and manually or automatically corrected. Customized Matlab code (The MathWorks Inc.) was used for automatic corrections.

\section{Modulation representation}

The modulation content of the stimuli was computed using a biologically inspired model of auditory processing (Chi et al., 2005). The auditory model consists of two main components: an early stage that accounts for the transformations that acoustic signals undergo in the early auditory system, from the cochlea to the midbrain; and a cortical stage that simulates the processing of the acoustic input at the level of the (primary) auditory cortex (see Chapter 1 for a detailed description of the model). We derived the auditory spectrogram and its modulation content using the "NSL Tools" package (available at http://www.isr.umd.edu/Labs/NSL/Software.htm) and customized Matlab code (The MathWorks Inc.). Sounds' spectrograms were generated using a bank of 128 overlapping bandpass filters with constant- $\mathrm{Q}\left(\mathrm{Q}_{10 \mathrm{~dB}}=3\right)$ mimicking the auditory periphery. Filters were equally spaced along a logarithmic frequency axis and covered a range of 5.3 oct ( $f=180-7040 \mathrm{~Hz}$ ). The output of the filter bank underwent band-pass filtering (hair cell stage), first-order derivative with respect to the frequency axis, half-wave rectification, and short-term temporal integration with time constant $\tau=8 \mathrm{~ms}$ (midbrain stage). The modulation content of the auditory spectrogram was computed through a bank of 2D modulation selective filters with joint selectivity for spectral and temporal modulations (see Joint frequency-specific MTF-based model, Chapter 2). These filters have constant $\mathrm{Q}$ and are directional, i.e. they respond either to upward or downward frequency sweeps. Filters were tuned to 6 spectral modulation frequencies $(\Omega=[0.5,0.7,1.1,1.7,2.6,4]$ cyc/oct), and 10 temporal modulation frequencies $(\omega=[1,1.4,2.1,3.1,4.5,6.6,9.7,14$, $20.6,30] \mathrm{Hz}$ ). The filter bank output was computed at each frequency along the tonotopic axis and then averaged over time. In order to decrease the computational load, a reduced modulation representation was obtained as follows. First, the time-averaged output of the filter bank was averaged across the upward 
and downward filter directions. Then, we divided the tonotopic axis in 60 ranges with constant bandwidth in octaves and averaged the modulation energy within each of these regions. This resulted in a representation with 6 spectral modulation frequencies $\times 10$ temporal modulation frequencies $\times 60$ frequencies $=3600$ features (i.e. 3600 reconstruction models to estimate). The above processing steps were applied to all stimuli, resulting into an $[N \times F]$ feature matrix $\mathbf{S}$ of average modulation energy, where $N$ is the number of sounds, and $F$ is the number of features in the reduced modulation representation.

\section{Estimation of $\mathrm{fMRI}$ responses to natural sounds}

We computed voxels' responses to natural sounds using customized Matlab code (The MathWorks Inc.). For each voxel $i$, the response vector $Y_{i}[(N \times 1), N=$ number of sounds] was obtained in two steps. First, a hemodynamic response function (HRF) common to all stimuli was estimated via a deconvolution analysis in which all stimuli were treated as a single condition. Then, using this HRF and one predictor per sound, we computed the beta weight of each sound (Kay et al., 2008). In Experiment 2, we implemented a 4-fold cross validation across the 4 stimulus sets (see above). The HRF was estimated using the training data and beta weights were computed separately for training and testing sounds. Further analyses were performed on voxels with a significant positive response to the training sounds $(p<0.05$, uncorrected in order not to be too stringent at this stage of the process) within an anatomically defined mask, which included HG, PT, PP, and STG.

\section{Estimation of model parameters}

We defined a reconstruction model as the linear relationship between patterns of $\mathrm{FMRI}$ activation within an $\mathrm{ROI}$ and the modulation representation. For each subject, independent reconstruction models were trained for every feature of the modulation space, as follows. The stimulus feature $S_{i}\left[N_{\text {train }} \times 1\right]$ was modeled as a linear transformation of the multivoxel response pattern $\mathbf{Y}_{\text {train }}\left[N_{\text {train }} \times V\right]$ plus a bias term $b_{i}$ and a noise term $n\left[N_{\text {train }} \times 1\right]$ as follows:

$$
S_{i}=\mathbf{Y}_{\text {train }} C_{i}+b_{i} 1+n
$$

where $N_{\text {train }}$ is the number of sounds in the training set, $V$ is the number of voxels, 1 is a $\left[N_{\text {train }} \times 1\right]$ vector of ones, and $C_{i}$ is a $[V \times 1]$ vector of model parameters, whose elements $c_{i j}$ quantify the contribution of voxel $j$ to the encoding of feature $i$. 
The solution to Equation 1 was computed using kernel ridge regression (Hoerl and Kennard, 1970; Bishop, 2006). The regularization parameter $\lambda$ was determined independently for each feature by generalized cross validation (Golub et al., 1979).

We implemented k-fold cross validation with 6 folds in Experiment 1 (120 training sounds, 24 testing sounds) and 4 folds in Experiment 2 (216 training sounds, 72 testing sounds). Note that in both experiments training and testing sets included different stimuli. Folds were generated in a pseudo-random manner in order to balance sound categories across folds. The same folds were used for all ROls.

Model evaluation: reconstruction accuracy and statistical analysis

Reconstructions from the $\mathrm{k}$ test sets were concatenated and reconstruction models were assessed individually by computing the Pearson's correlation coefficient ( $r$ ) between the reconstructed and the actual stimulus features. This resulted in 3600 correlation coefficients per ROI per hemisphere, which represented the $M_{T F}$ and $M T F_{L H}$ for a given ROI. For statistical testing, all correlation values were transformed into z-scores by applying the Fisher's ztransform. To assess the statistical significance of the contrasts $\mathrm{MTF}_{\mathrm{RH}}>$ chance and MTF ${ }_{L H}>$ chance, we pooled together results from the two experiments and implemented a non-parametric random-effects group analysis for every feature, as follows. First, for each stimulus feature we computed the null distribution of correlation coefficients at the single-subject level. Null distributions were obtained by randomly permuting (500 times) the stimulus labels of the reconstructed features and computing the correlation coefficient for each permutation. The empirical chance level of correlation $r_{\text {chance }}$ was defined as the mean of the null distribution. For each feature, we then performed a random-effects 1-tailed nonparametric test (exact permutation test) to assess whether the correlation between reconstructed and original feature was significantly higher than chance at the group level (note that negative correlations are not meaningful because they indicate poor reconstruction). Our test statistic was the group average of the individual differences $d=r-r_{\text {chance. }}$. To obtain the null distribution of the group average difference, we changed the sign of $d$ for a randomly selected subset of subjects and recomputed the group average (Menke and Martinez, 2004). This procedure was repeated for all possible permutations of sign change $\left(2^{7}=128\right)$ and the $p$ value was computed as the proportion of permutations that yielded a test statistic equal or more extreme than the observed one. In order to correct for 
multiple comparisons across stimulus features, we employed a cluster-size threshold procedure (Forman et al., 1995). For this, cluster-level false-positive rates were estimated for every permutation using an initial uncorrected threshold of 0.05. The minimum cluster-size threshold which yielded a cluster-level falsepositive rate (alpha) of $5 \%$ was then applied to the MTFs. The statistical procedure described above was applied to all ROls independently.

In the cases where no statistically significant inter-hemispheric differences were observed (see below), MTFs were averaged across hemispheres and the same procedure described above was used to test the statistical significance of the average MTF (MTF average>chance). The empirical chance level of correlation was computed as the average between the chance levels of individual hemispheres (see above).

Post hoc statistical analysis of MTFs' marginal profiles

MTFs' marginal profiles were obtained by averaging the group-averaged MTFs along irrelevant dimensions (e.g. frequency and temporal modulation for the spectral modulation profile). To assess the statistical significance of the MTFs' profiles, we performed the following post hoc analyses. For the spectral modulation profile, we compared the reconstruction accuracy at 0.5 cyc/oct $\left(r_{0.5}\right)$ with the accuracy at 4 cyc/oct $\left(r_{4}\right)$. Our test statistic was the average across frequency and temporal modulations of the differences $d=r_{0.5}-r_{4}$. To obtain the null distribution of the average difference, we changed the sign of $d$ for a randomly selected subset of points and recomputed the average. This procedure was repeated 2000 times and the $p$ value was computed as the proportion of permutations that yielded a test statistic equal or more extreme than the observed one (in absolute values). A similar statistical procedure was applied to all post hoc tests of the MTFs' profiles (see ROl-based analysis of spectral and temporal response properties).

Analysis of inter-hemispheric and regional differences

To evaluate inter-hemispheric differences between corresponding ROls, we performed the following conjunction analyses: $\left(M T F_{R H}>M T F_{L H}\right) \cap\left(M T F_{R H}>\right.$ chance), and $\left(M T F_{L H}>M T F_{R H}\right) \cap\left(M T F_{L H}>\right.$ chance). For each ROI, we assessed the statistical significance of the contrasts $M T F_{R H}>M T F_{L H}$ and $M T F_{L H}>M T F_{R H}$ by performing a random-effects 1-tailed non-parametric test (exact permutation test) for each contrast. For every feature, we computed the group average of the individual 
difference $d$, which was defined as $d=r_{R H}-r_{L H}$ for the contrast $M T F_{R H}>M T F_{L H}$ and $d=$ $r_{L H}-r_{R H}$ for the contrast $M T F_{L H}>M T F_{R H}$. To obtain the null distribution of the group average difference, we changed the sign of $d$ for a randomly selected subset of subjects and recomputed the group average. This procedure was repeated for all possible permutations of sign change $\left(2^{7}=128\right)$ and the $p$ value was computed as the proportion of null values equal or more extreme than the observed group average difference. In order to correct for false positives, we employed a clustersize threshold procedure (Forman et al., 1995). For every permutation, we estimated cluster-level false-positive rates using an initial uncorrected threshold of 0.05. Finally, we applied the minimum cluster-size threshold which yielded a cluster-level false-positive rate (alpha) of $5 \%$.

To test regional differences between MTF, we carried out all possible pairwise comparisons between ROls. For every pair $\mathrm{MTF}_{\mathrm{i}}$ and $\mathrm{MTF}_{\mathrm{j}}$, we performed the following conjunction analyses: $\left(M_{T F}>M T F_{j}\right) \cap\left(M T F_{i}>\right.$ chance), and $\left(M_{T} F_{j}>M T F_{i}\right) \cap$ $\left(M_{T F}>\right.$ chance). Contrasts $M T F_{i}>M T F_{j}$ and $M T F_{j}>M T F_{i}$ were tested using the same procedure described above for the inter-hemispheric differences.

\section{References}

Ahveninen J, Jääskeläinen $I P$, Raij $T$, Bonmassar $G$, Devore $S$, Hämäläinen $M$, Levänen S, Lin F-H, Sams M, Shinn-Cunningham BG, Witzel T, Belliveau JW (2006) Task-modulated "what" and "where" pathways in human auditory cortex. Proc Natl Acad Sci U S A 103:14608-14613.

Altmann CF, Bledowski C, Wibral M, Kaiser J (2007) Processing of location and pattern changes of natural sounds in the human auditory cortex. Neuroimage 35:1192-1200.

Barton B, Venezia J (2012) Orthogonal acoustic dimensions define auditory field maps in human cortex. Proc Natl Acad Sci U S A 109:20738-20743.

Belin P, Zatorre RJ, Lafaille P, Ahad P, Pike B (2000) Voice-selective areas in human auditory cortex. Nature 403:309-312.

Bialek W, Rieke F, de Ruyter van Steveninck RR, Warland D (1991) Reading a neural code. Sci $252: 1854-1857$.

Bishop C (2006) Pattern Recognition and Machine Learning. New York: Springer. 
Bitterman Y, Mukamel R, Malach R, Fried I, Nelken I (2008) Ultra-fine frequency tuning revealed in single neurons of human auditory cortex. Nature 451:197201.

Bonte M, Valente G, Formisano E (2009) Dynamic and task-dependent encoding of speech and voice by phase reorganization of cortical oscillations. J Neurosci 29:1699-1706.

Chi T, Ru P, Shamma S (2005) Multiresolution spectrotemporal analysis of complex sounds. J Acoust Soc Am 118:887-906.

Da Costa S, van der Zwaag W, Marques JP, Frackowiak RSJ, Clarke S, Saenz M (2011) Human primary auditory cortex follows the shape of Heschl's gyrus. J Neurosci 31:14067-14075.

De Martino F, Moerel M, van de Moortele P-F, Ugurbil K, Goebel R, Yacoub E, Formisano $E$ (2013) Spatial organization of frequency preference and selectivity in the human inferior colliculus. Nat Commun 4:1386.

deCharms RC (1998) Optimizing Sound Features for Cortical Neurons. Science (80) 280:1439-1444.

Deistung A, Schäfer A, Schweser F, Biedermann U, Turner R, Reichenbach JR (2013) Toward in vivo histology: a comparison of quantitative susceptibility mapping (OSM) with magnitude-, phase-, and R2*-imaging at ultra-high magnetic field strength. Neuroimage 65:299-314.

Di Salle F, Formisano E, Seifritz E, Linden DE, Scheffler K, Saulino C, Tedeschi G, Zanella FE, Pepino A, Goebel R, Marciano E (2001) Functional fields in human auditory cortex revealed by time-resolved $\mathrm{FMRI}$ without interference of EPI noise. Neuroimage 13:328-338.

Elliott TM, Theunissen FE (2009) The modulation transfer function for speech intelligibility. PLoS Comput Biol 5:e1000302.

Forman SD, Cohen JD, Fitzgerald M, Eddy WF, Mintun M a, Noll DC (1995) Improved assessment of significant activation in functional magnetic resonance imaging (fMRI): use of a cluster-size threshold. Magn Reson Med 33:636-647.

Formisano E, De Martino F, Bonte M, Goebel R (2008) "Who" Is Saying "What"? Brain-Based Decoding of Human Voice and Speech. Sci 322 :970-973. 
Formisano E, Kim DS, Di Salle F, van de Moortele PF, Ugurbil K, Goebel R (2003) Mirror-symmetric tonotopic maps in human primary auditory cortex. Neuron 40:859-869.

Gaab N, Gabrieli JDE, Glover GH (2007) Assessing the influence of scanner background noise on auditory processing. I. An fMRI study comparing three experimental designs with varying degrees of scanner noise. Hum Brain Mapp 28:703-720.

Geyer S, Weiss M, Reimann K, Lohmann G, Turner R (2011) Microstructural Parcellation of the Human Cerebral Cortex - From Brodmann's Post-Mortem Map to in vivo Mapping with High-Field Magnetic Resonance Imaging. Front Hum Neurosci 5:19.

Giraud AL, Lorenzi C, Ashburner J, Wable J, Johnsrude I, Frackowiak R, Kleinschmidt A (2000) Representation of the temporal envelope of sounds in the human brain. J Neurophysiol 84:1588-1598.

Goebel R, Esposito F, Formisano E (2006) Analysis of functional image analysis contest (FIAC) data with brainvoyager QX: From single-subject to cortically aligned group general linear model analysis and self-organizing group independent component analysis. Hum Brain Mapp 27:392-401.

Golub G, Heath M, Wahba G (1979) Generalized Cross-Validation as a Method for Choosing a Good Ridge Parameter. Technometrics 21:215-223.

Haxby J V, Gobbini MI, Furey ML, Ishai A, Schouten JL, Pietrini P (2001) Distributed and overlapping representations of faces and objects in ventral temporal cortex. Science 293:2425-2430.

Herdener M, Esposito F, Scheffler K, Schneider P, Logothetis NK, Uludag K, Kayser C (2013) Spatial representations of temporal and spectral sound cues in human auditory cortex. Cortex 49:2822-2833.

Hoerl AE, Kennard RW (1970) Ridge Regression: Biased Estimation for Nonorthogonal Problems. Technometrics 12:55-67.

Kay KN, David S V, Prenger RJ, Hansen KA, Gallant JL (2008) Modeling lowfrequency fluctuation and hemodynamic response timecourse in eventrelated fMRI. Hum Brain Mapp 29:142-156. 
Kim J-J, Crespo-Facorro B, Andreasen NC, O'Leary DS, Zhang B, Harris G, Magnotta VA (2000) An MRI-Based Parcellation Method for the Temporal Lobe. Neuroimage 11:271-288.

Klein DJ, König P, Körding KP (2003) Sparse Spectrotemporal Coding of Sounds. EURASIP J Adv Signal Process 2003:659-667.

Kleinschmidt M (2002) Robust speech recognition based on spectro-temporal processing. PhD Thesis, University Oldenbg.

Kowalski N, Depireux DA, Shamma SA (1996) Analysis of dynamic spectra in ferret primary auditory cortex. II. Prediction of unit responses to arbitrary dynamic spectra. J Neurophysiol 76:3524-3534.

Langers DRM, Backes WH, Dijk P Van (2003) Spectrotemporal features of the auditory cortex: the activation in response to dynamic ripples. Neuroimage 20:265-275.

Langers DRM, Backes WH, van Dijk P (2007) Representation of lateralization and tonotopy in primary versus secondary human auditory cortex. Neuroimage 34:264-273.

Laudanski J, Edeline J-M, Huetz C (2012) Differences between Spectro-Temporal Receptive Fields Derived from Artificial and Natural Stimuli in the Auditory Cortex. PLoS One 7:e50539.

Leaver AM, Rauschecker JP (2010) Cortical representation of natural complex sounds: effects of acoustic features and auditory object category. J Neurosci 30:7604-7612.

Lewis JW, Brefczynski JA, Phinney RE, Janik JJ, DeYoe EA (2005) Distinct cortical pathways for processing tool versus animal sounds. J Neurosci 25:51485158.

Lewis JW, Talkington WJ, Walker NA, Spirou GA, Jajosky A, Frum C, BrefczynskiLewis $J$ a (2009) Human cortical organization for processing vocalizations indicates representation of harmonic structure as a signal attribute. J Neurosci 29:2283-2296.

Machens CK, Gollisch T, Kolesnikova O, Herz AVM (2005) Testing the efficiency of sensory coding with optimal stimulus ensembles. Neuron 47:447-456. 
Menke J, Martinez TR (2004) Using permutations instead of student's t distribution for p-values in paired-difference algorithm comparisons. Neural Networks, 2004 Proceedings 2004 IEEE Int Jt Conf 2:1331-1335 vol.2.

Mesgarani N, Chang EF (2012) Selective cortical representation of attended speaker in multi-talker speech perception. Nature:1-5.

Mesgarani N, David S V, Fritz JB, Shamma S a (2009) Influence of context and behavior on stimulus reconstruction from neural activity in primary auditory cortex. J Neurophysiol 102:3329-3339.

Miyawaki Y, Uchida H, Yamashita O, Sato M, Morito Y, Tanabe HC, Sadato N, Kamitani $Y$ (2008) Visual image reconstruction from human brain activity using a combination of multiscale local image decoders. Neuron 60:915-929.

Moerel M, De Martino F, Formisano E (2012) Processing of natural sounds in human auditory cortex: tonotopy, spectral tuning, and relation to voice sensitivity. J Neurosci 32:14205-14216.

Moerel M, De Martino F, Santoro R, Ugurbil K, Goebel R, Yacoub E, Formisano E (2013) Processing of natural sounds: characterization of multipeak spectral tuning in human auditory cortex. J Neurosci 33:11888-11898.

Morosan P, Rademacher J, Schleicher A, Amunts K, Schormann T, Zilles K (2001) Human primary auditory cortex: cytoarchitectonic subdivisions and mapping into a spatial reference system. Neuroimage 13:684-701.

Morosan P, Schleicher A, Amunts K, Zilles K (2005) Multimodal architectonic mapping of human superior temporal gyrus. Anat Embryol:401-406.

Pasley BN, David S V, Mesgarani N, Flinker A, Shamma S, Crone NE, Knight RT, Chang EF (2012) Reconstructing Speech from Human Auditory Cortex Zatorre R, ed. PLoS Biol 10:e1001251.

Poeppel D (2003) The analysis of speech in different temporal integration windows: cerebral lateralization as "asymmetric sampling in time." Speech Commun 41:245-255.

Santoro R, Moerel M, De Martino F, Goebel R, Ugurbil K, Yacoub E, Formisano E (2014) Encoding of Natural Sounds at Multiple Spectral and Temporal Resolutions in the Human Auditory Cortex. PLoS Comput Biol 10:e1003412. 
Schönwiesner M, Rübsamen R, von Cramon DY (2005) Hemispheric asymmetry for spectral and temporal processing in the human antero-lateral auditory belt cortex. Eur J Neurosci 22:1521-1528.

Schönwiesner M, Zatorre RJ (2009) Spectro-temporal modulation transfer function of single voxels in the human auditory cortex measured with high-resolution fMRI. Proc Natl Acad Sci U S A 106:14611-14616.

Singh NC, Theunissen FE (2003) Modulation spectra of natural sounds and ethological theories of auditory processing. J Acoust Soc Am 114:3394-3411.

Staeren N, Renvall H, De Martino F, Goebel R, Formisano E (2009) Sound categories are represented as distributed patterns in the human auditory cortex. Curr Biol 19:498-502.

Stanley GB, Li FF, Dan Y (1999) Reconstruction of natural scenes from ensemble responses in the lateral geniculate nucleus. J Neurosci 19:8036-8042.

Theunissen E, Sen K, Doupe AJ (2000) Spectral-Temporal Receptive Fields of Nonlinear Auditory Neurons. J Neurosci 20:2315-2331.

Van de Moortele P-F, Auerbach EJ, Olman C, Yacoub E, Uğurbil K, Moeller S (2009) T1 weighted brain images at 7 Tesla unbiased for Proton Density, T2* contrast and RF coil receive $\mathrm{B} 1$ sensitivity with simultaneous vessel visualization. Neuroimage 46:432-446.

Wallace MN, Johnston PW, Palmer AR (2002) Histochemical identification of cortical areas in the auditory region of the human brain. Exp brain Res 143:499-508.

Warrier C, Wong P, Penhune V, Zatorre R, Parrish T, Abrams D, Kraus N (2009) Relating structure to function: Heschl's gyrus and acoustic processing. J Neurosci 29:61-69.

Woolley SMN, Fremouw TE, Hsu A, Theunissen FE (2005) Tuning for spectrotemporal modulations as a mechanism for auditory discrimination of natural sounds. Nat Neurosci 8:1371-1379.

Zatorre RJ, Belin P (2001) Spectral and Temporal Processing in Human Auditory Cortex. Cereb Cortex 11 :946-953. 


\section{Supporting Information}

A

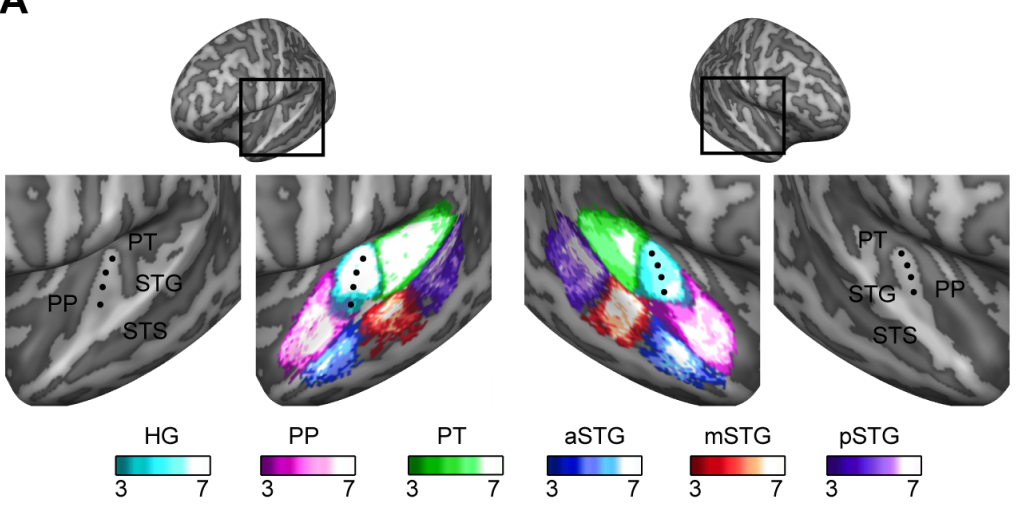

B
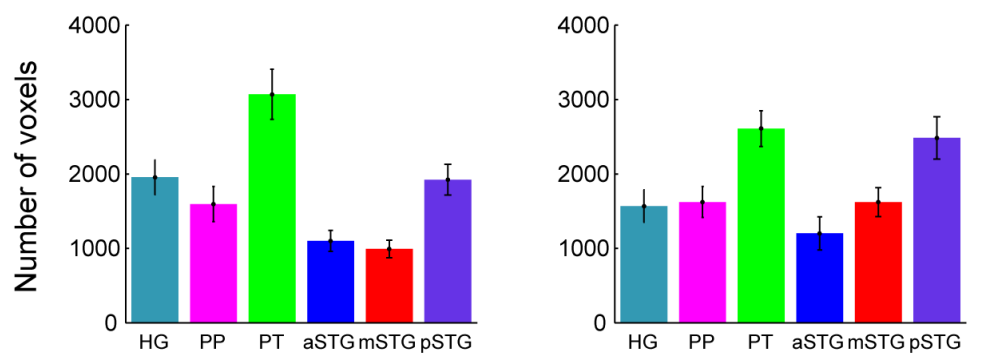

C
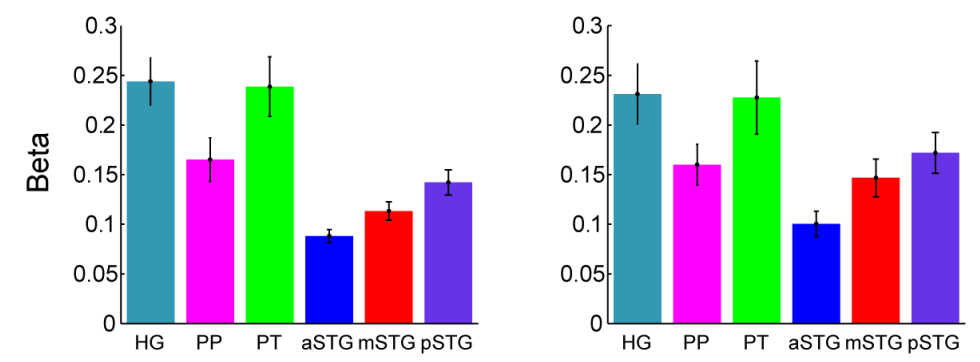

Figure S3.1 Anatomical ROIs. (A) Top: inflated representation of the group cortical surface mesh. ROls are shown in the cortical region highlighted by the black square. Bottom: Overlap of defined regions across three to seven subjects. The black dots indicate HG. (B) Number of voxels selected in each ROI (mean \pm SEM, $N=7$ ) (C) Average response of selected voxels (mean $\pm S E M, N=7$ ). 


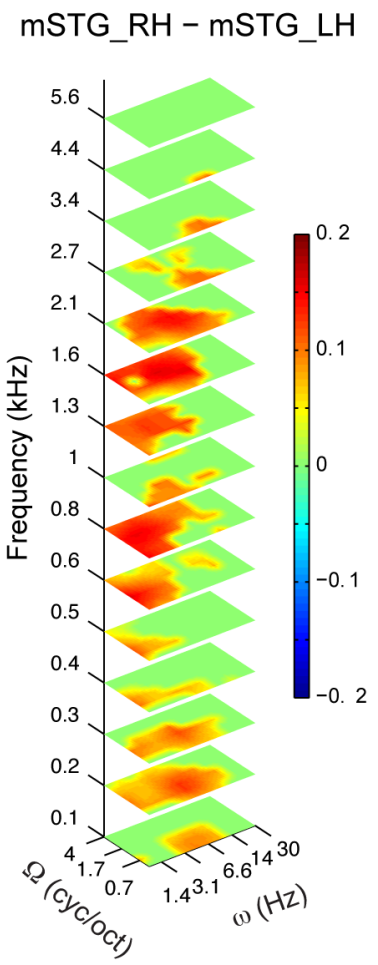

Figure S3.2. Hemispheric differences. Group-averaged difference between the MTFs of the right and left mSTG. Features that are not significantly different are assigned with 0 and depicted in green (see Material and Methods for details of the statistical analysis). MTFs have been interpolated for display purposes. $\Omega$ : spectral modulation frequency; $\omega$ : temporal modulation frequency. 
$H G$

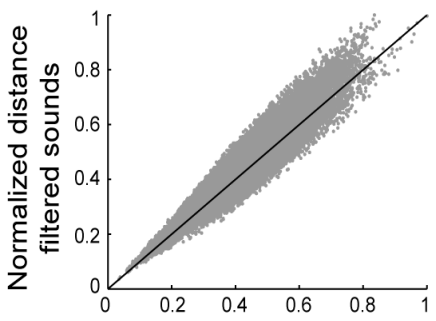

aSTG

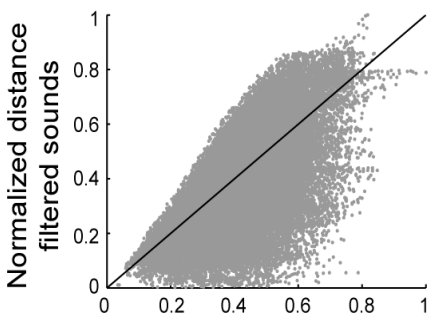

pSTG

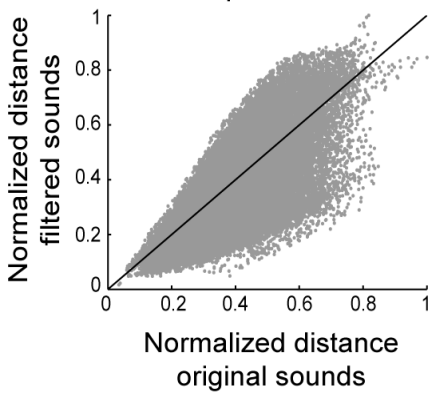

PP

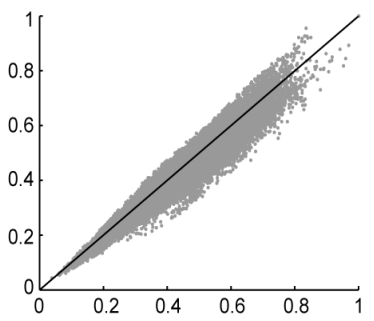

mSTG_RH

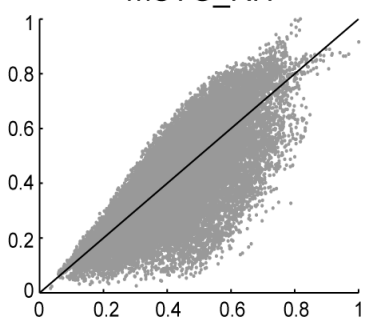

PT

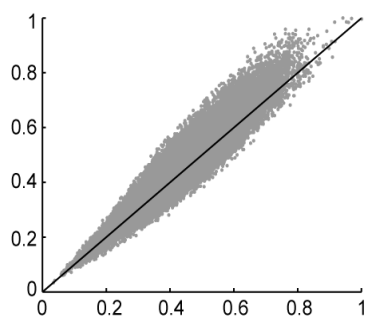

MSTG_LH

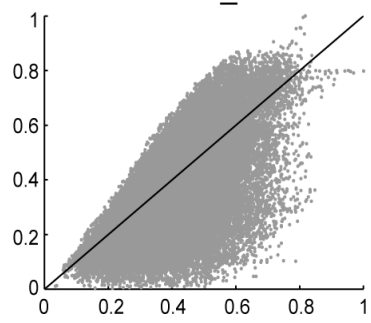

Figure S3.3. Discriminability analysis of the entire stimulus set. Each panel shows the relation between normalized pairwise distances of the stimuli in the original acoustic space and after filtering them with the MTF of one ROI. The solid line represents the diagonal. Points lying on the diagonal designate pairs of sounds whose normalized relative distance does not change after the filtering operation. A shift above (below) the diagonal indicates that the filtering operation increases (decreases) the normalized relative distances between sounds. 
HG

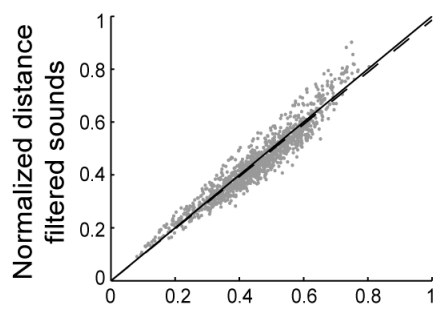

aSTG

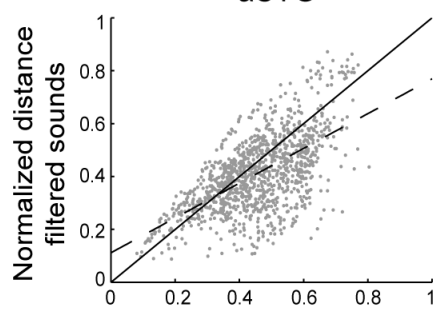

pSTG

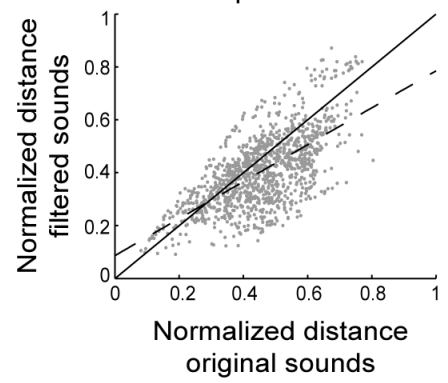

PT
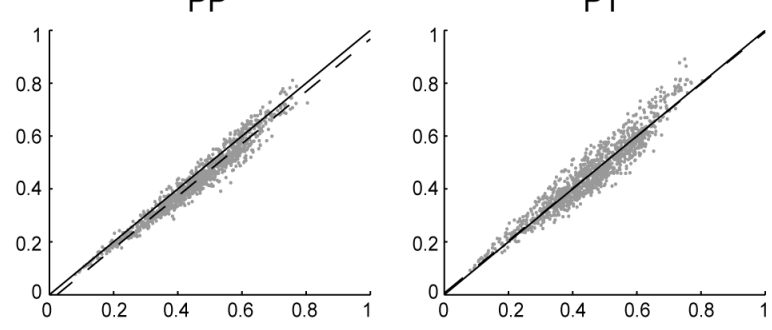

mSTG_RH

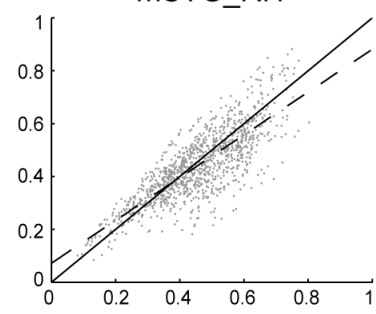

mSTG_LH

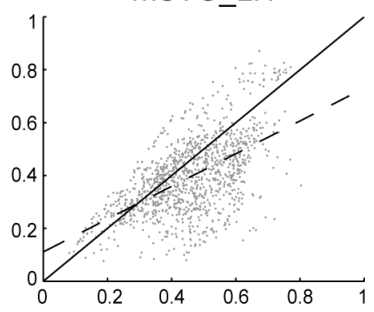

Figure S3.4. Discriminability analysis of music sounds. Each panel shows the relation between normalized pairwise distances of the stimuli in the original acoustic space and after filtering them with the MTF of one ROI. The dashed line is the fitted line and the solid line represents the diagonal. 
HG

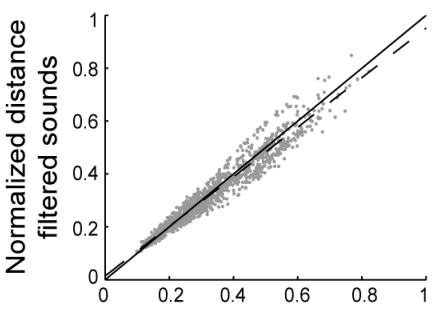

aSTG

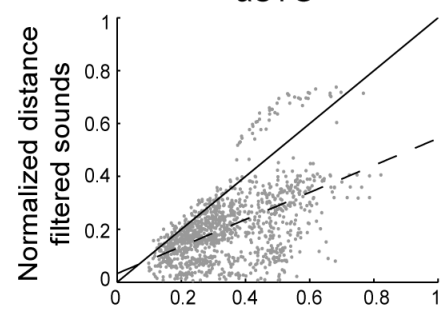

pSTG

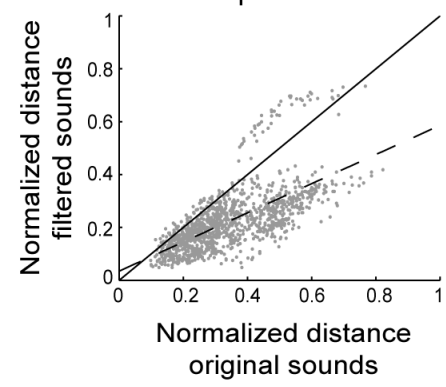

PP

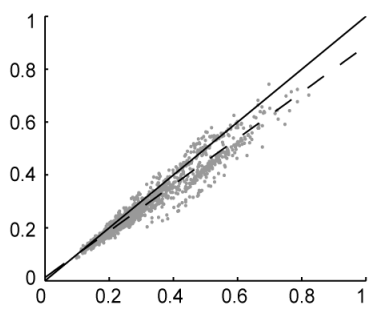

mSTG_RH

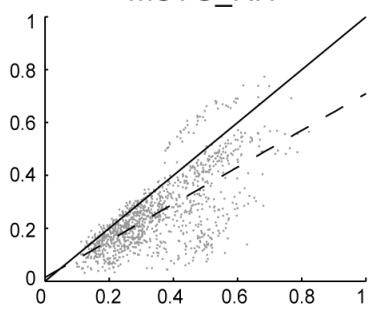

PT

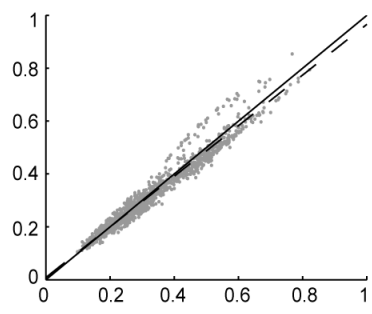

mSTG_LH

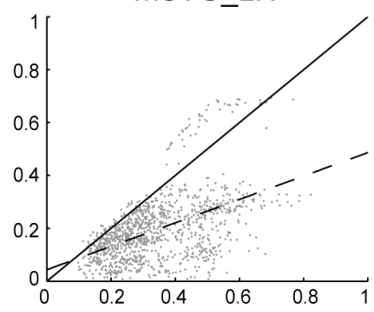

Figure S3.5. Discriminability analysis of tools sounds. Each panel shows the relation between normalized pairwise distances of the stimuli in the original acoustic space and after filtering them with the MTF of one ROI. The dashed line is the fitted line and the solid line represents the diagonal. 
HG

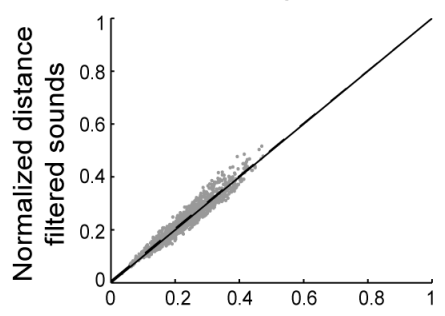

aSTG

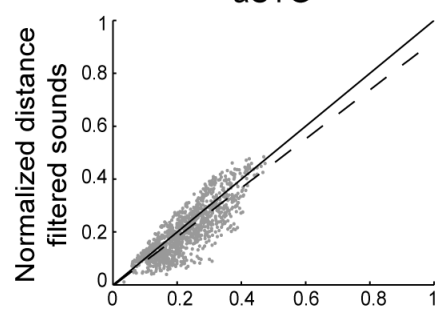

pSTG

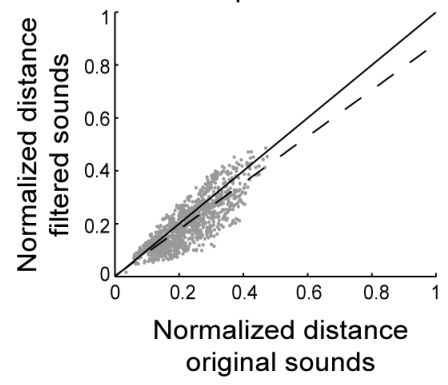

PT
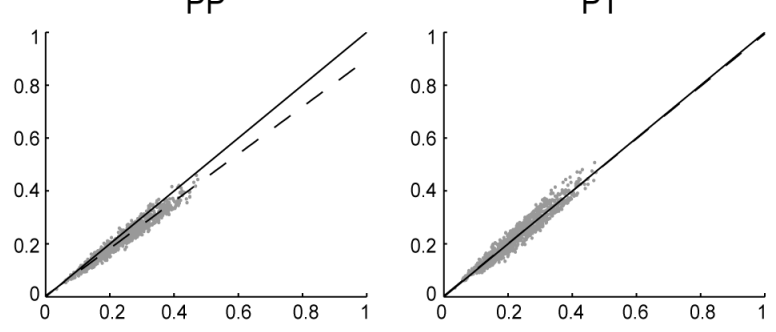

mSTG_RH

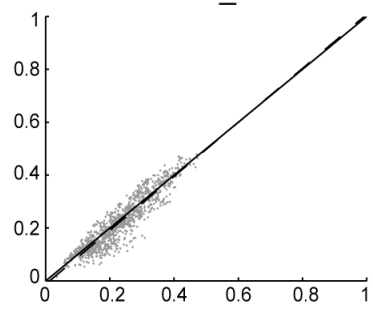

mSTG_LH

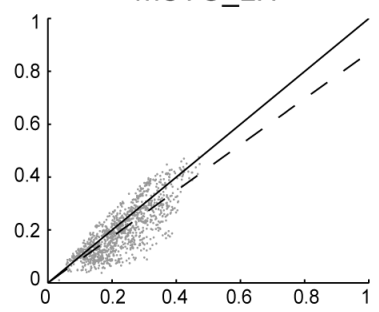

Figure S3.6. Discriminability analysis of nature sounds. Each panel shows the relation between normalized pairwise distances of the stimuli in the original acoustic space and after filtering them with the MTF of one ROI. The dashed line is the fitted line and the solid line represents the diagonal. 
HG

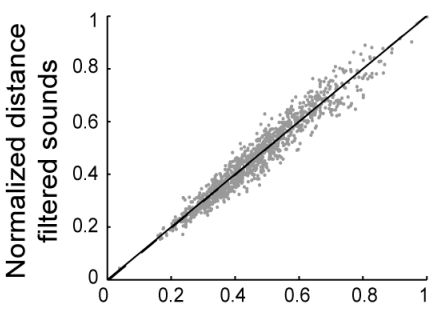

aSTG

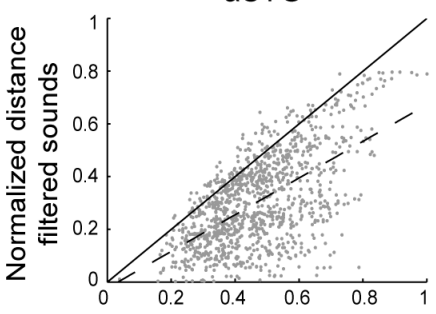

pSTG

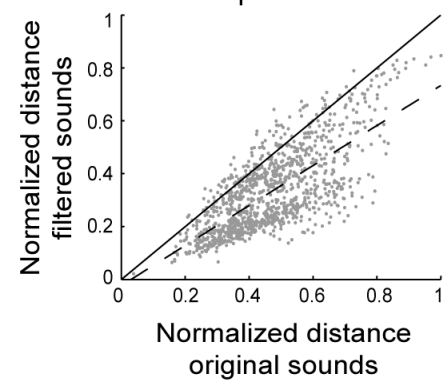

PP

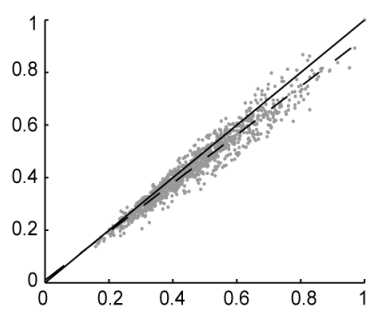

mSTG_RH

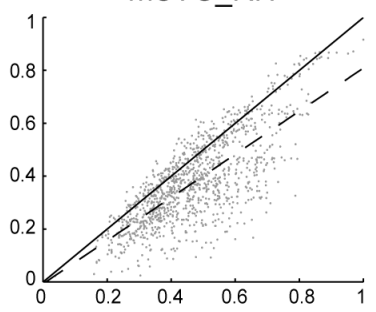

PT

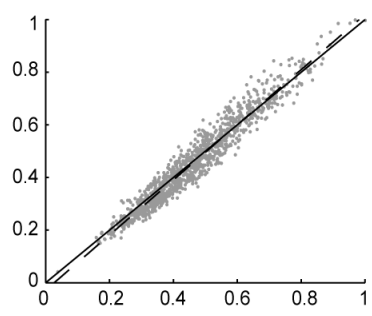

mSTG_LH

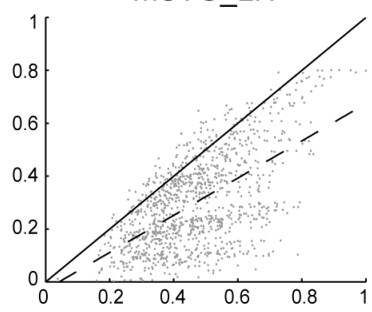

Figure S3.7. Discriminability analysis of animal sounds. Each panel shows the relation between normalized pairwise distances of the stimuli in the original acoustic space and after filtering them with the MTF of one ROI. The dashed line is the fitted line and the solid line represents the diagonal. 
HG

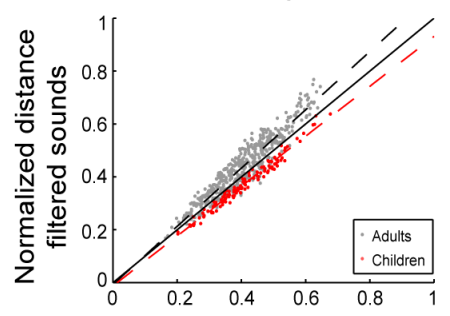

aSTG

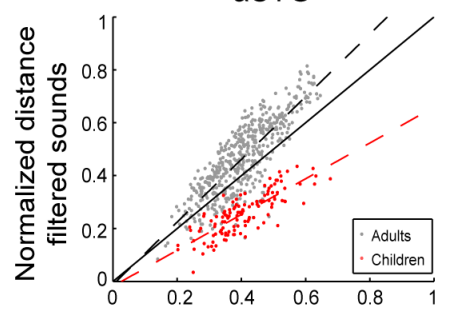

pSTG

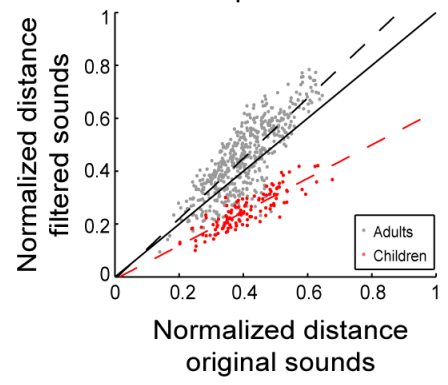

PP

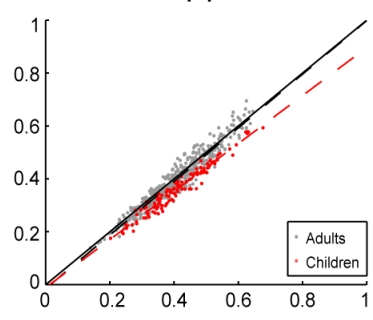

mSTG_RH

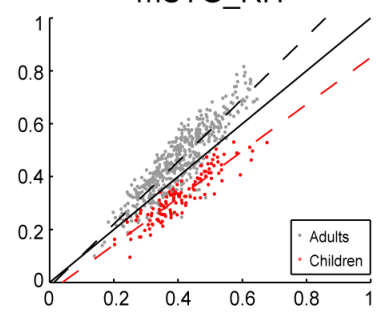

PT

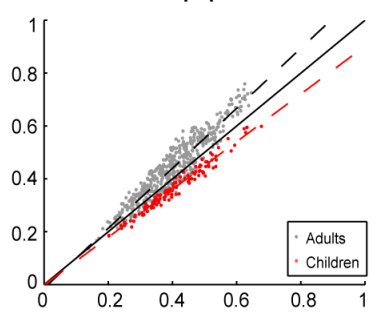

mSTG_LH

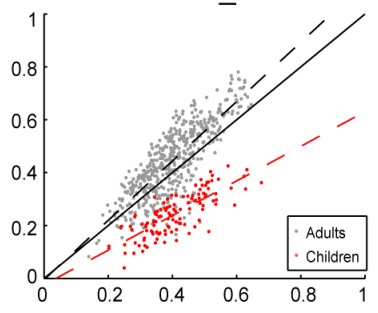

Figure S3.8. Discriminability analysis of vocal sounds. Each panel shows the relation between normalized pairwise distances of the stimuli in the original acoustic space and after filtering them with the MTF of one ROI. Adults' (grey) and children's (red) voices have been analyzed separately. The dashed lines are the fitted lines (see Table ST3.1 and ST3.2) and the solid line represents the diagonal. 


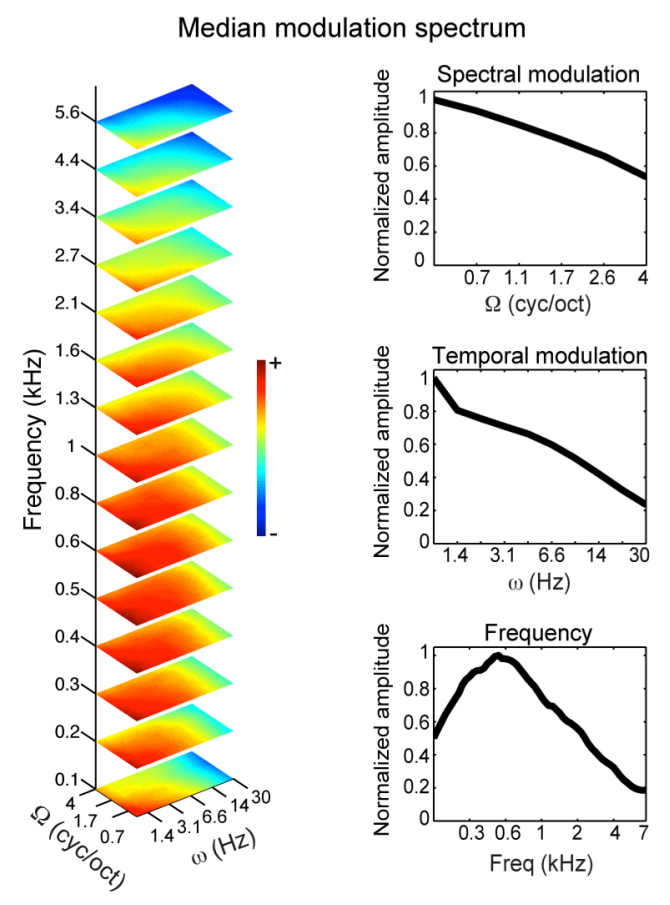

Figure S3.9. Modulation spectrum of the stimuli. Left: median modulation content of the stimuli as a function of frequency, spectral and temporal modulation. Values are shown using a logarithmic color scale, with red depicting the spectrotemporal modulations with the highest energy. Right: marginal profiles of the median modulation spectrum. 

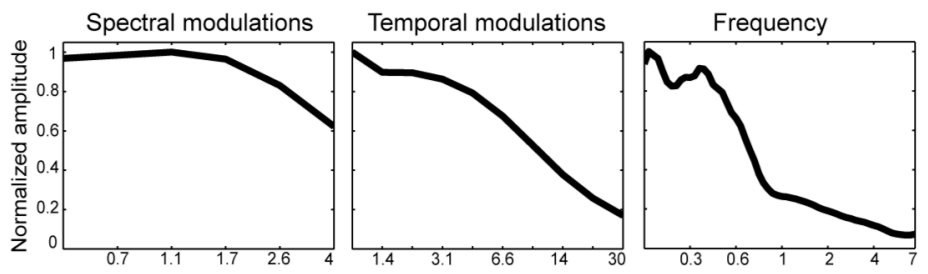

Speech
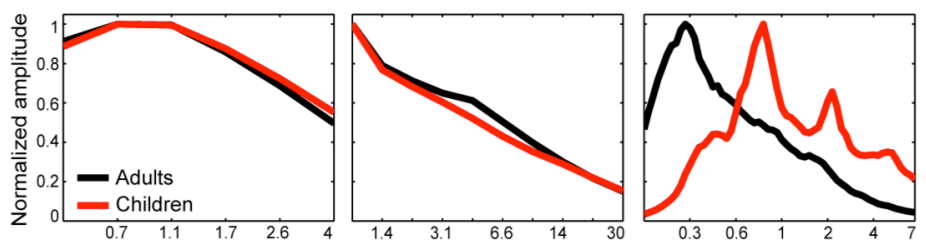

Voice
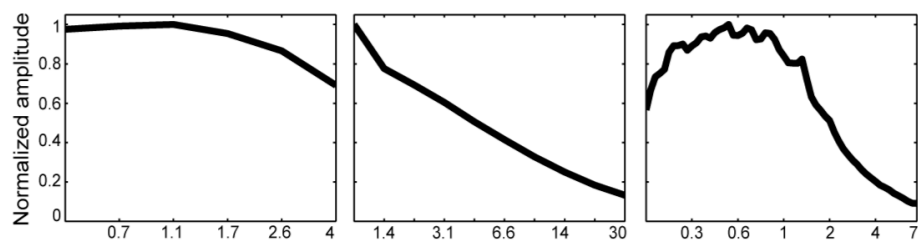

Music
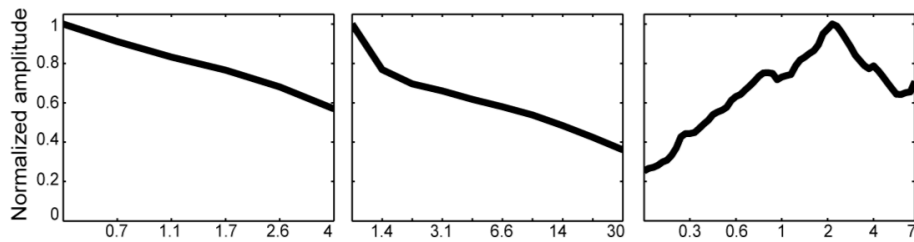

Tools
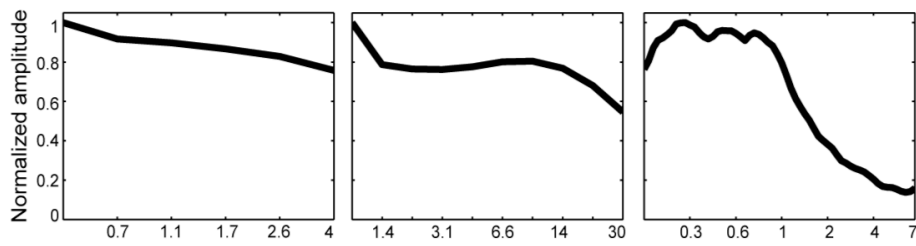

Nature
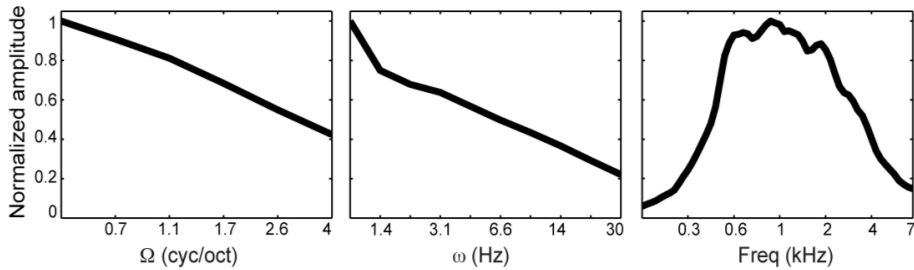

Animals

Figure S3.10. Marginal profiles of the modulation spectrum of individual categories. Each row illustrates the spectral (left), temporal (middle) and frequency (right) marginal profile of the median modulation content for one of the six sound categories that were equally represented in our stimulus set. Each profile has been normalized by its maximum value. 
Table ST3.1. Results of linear regression analysis for pairs of adults' vocal sounds.

\begin{tabular}{lcccccc}
\hline & $a$ & $S E_{a}$ & $b$ & $S E_{b}$ & $R^{2}$ & $F(1,494)$ \\
\hline HG & $1.10^{\star}$ & 0.02 & -0.01 & 0.01 & 0.89 & $3.96 \times 10^{3 \star}$ \\
PP & $1.01^{\star}$ & 0.01 & -0.02 & 0.01 & 0.93 & $6.81 \times 10^{3 \star}$ \\
PT & $1.13^{\star}$ & 0.02 & -0.01 & 0.01 & 0.89 & $3.83 \times 10^{3 \star}$ \\
aSTG & $1.18^{\star}$ & 0.04 & -0.01 & 0.01 & 0.68 & $1.07 \times 10^{3 *}$ \\
mSTG_RH & $1.18^{\star}$ & 0.03 & -0.02 & 0.01 & 0.80 & $1.95 \times 10^{3 \star}$ \\
mSTG_LH & $1.12^{\star}$ & 0.04 & -0.01 & 0.01 & 0.67 & $9.92 \times 10^{2 \star}$ \\
pSTG & $1.14^{\star}$ & 0.04 & -0.01 & 0.01 & 0.67 & $1.01 \times 10^{3 \star}$ \\
\hline
\end{tabular}

Regression coefficient ( $a=$ slope; $b=$ intercept), standard errors ( $\mathrm{SE}_{a}$ and $\mathrm{SE}_{\mathrm{b}}$ ), coefficient of determination $\left(R^{2}\right)$, and corresponding $F$ value. *Bonferroni adjusted $p<$ 0.001 (two tailed t test for the regression coefficients and $\mathrm{F}$ test for the $R^{2}$ ).

Table ST3.2. Pairwise comparisons of linear regression slopes for adults' vocal sounds.

\begin{tabular}{lcccccc}
\hline & PP & PT & aSTG & mSTG_RH & mSTG_LH & PSTG \\
\hline HG & $4.11^{\star}$ & -1.31 & -2.08 & -2.51 & -0.54 & -0.98 \\
PP & 0 & $-5.48^{*}$ & $-4.48^{*}$ & $-5.7^{\star}$ & -2.9 & $-3.34^{*}$ \\
PT & 0 & 0 & -1.24 & -1.45 & 0.29 & -0.15 \\
aSTG & 0 & 0 & 0 & 0.08 & 1.22 & 0.87 \\
mSTG_RH & 0 & 0 & 0 & 0 & 1.32 & 0.92 \\
mSTG_LH & 0 & 0 & 0 & 0 & 0 & -0.35 \\
\hline
\end{tabular}

z-scores of pairwise differences between linear regression slopes. Each cell represents the difference between the regression slope of the ROI listed in the row and the regression slope of the $\mathrm{ROI}$ listed in the column. *Bonferroni adjusted two tailed $\mathrm{p}<$ 0.001 . 


\section{Chapter 4}

\section{Re-synthesis of real-life sounds from non-invasively measured auditory cortical response patterns}

Corresponding manuscript (submitted):

Santoro R*, Moerel M, De Martino F, Valente G, Ugurbil K, Yacoub E, Formisano $E^{\star}$. Re-synthesis of real-life sounds from non-invasively measured auditory cortical response patterns

${ }^{*}$ Authors share equal contribution 


\section{Abstract}

Can we reconstruct the spectrograms (and waveforms) of complex sounds from measurements of listeners' brain responses? The accuracy with which we can answer this question provides an objective assessment of our current understanding of sound encoding in the human brain. For speech tokens, brainbased sound reconstruction has been shown feasible using signals invasively collected with intracranial electro-corticography. Here we show accurate spectrogram reconstruction of arbitrary real-life sounds (including human speech and voice, animal cries, and tool sounds) through computational modeling of brain responses non-invasively measured using 7 Tesla functional MRI (fMRI). We model high-spatial resolution fMRI response patterns based on the hypothesis that the auditory cortex forms multiple views of a sound spectrogram at different spectral and temporal resolutions. The accuracy of re-constructed spectrograms - in spite of the poor temporal resolution of $\mathrm{fMRI}$ - demonstrates the validity of our modeling hypothesis. Our results reveal a relevant computational mechanism that the human brain uses for encoding sounds. Future extension of this research to the mental imagery of sounds may lead to new brain computer interfaces and clinical applications (e.g. "listening" of auditory hallucinations). 


\section{Introduction}

A screaming voice, the singing of a bird, the roaring of a car's engine. These are just a few examples illustrating the diversity of sounds we encounter in our everyday life. How does the auditory system deal with the variety and complexity of real-life, natural sounds? At the sensory periphery (cochlea), the representation of incoming sounds can be faithfully described by their spectrogram, a twodimensional image that depicts the combined spectral and temporal variations of acoustic energy (Figure 1.1). Spectrograms of real-life sounds differ largely among each other, but in many cases they present characteristic temporal, spectral and spectro-temporal modulations (Singh and Theunissen, 2003) (Figure 1.2). Psychophysical studies suggest that these modulations convey relevant behavioral information, pivotal e.g. for sound recognition (Patil et al., 2012) and speech intelligibility (Drullman et al., 1994; Shannon et al., 1995; Chi et al., 1999; Elliott and Theunissen, 2009). However, how spectro-temporal modulations and - more generally - spectrograms of complex sounds are encoded in the human brain remains largely unknown.

In the present study, we combine high spatial resolution (7 Tesla) functional magnetic resonance imaging ( $\mathrm{fMRI}$ ) with the analytical approach of model-based stimulus reconstruction (Bialek et al., 1991; Stanley et al., 1999; Miyawaki et al., 2008; Mesgarani et al., 2009; Pasley et al., 2012) to investigate the representation of real-life sound spectrograms in the network of auditory cortical areas. Stimulus reconstruction represents the most sophisticated application of brain decoding. Unlike the more common classification-based decoding (which only allows discriminating between discrete levels of stimulus categories), stimulus reconstruction aims at retrieving - from brain response patterns - the accurate sensory build-up of individual, arbitrary stimuli. In combination with fMRI, stimulus reconstruction has been applied so far to the study of the visual system. In this context, researchers have been able to reconstruct simple geometric patterns and complex natural images in both static (Thirion et al., 2006; Miyawaki et al., 2008; Naselaris et al., 2009; Schoenmakers et al., 2013) and dynamic conditions (Nishimoto et al., 2011). In the auditory domain, stimulus reconstruction techniques have recently been used in combination with intracranial electrocorticography (ECoG) to investigate the representation of speech (Pasley et al., 2012), as well as the encoding of attended and ignored speech in the context of multiple, concurrent speakers (Mesgarani and Chang, 2012). More challenging is the reconstruction of the spectro-temporal dynamics of natural sounds from patterns of fMRI activity, as blood oxygen level-dependent (BOLD) signals measured with fMRI 
are very slow compared to the fast temporal changes that characterize the spectrogram of natural sounds. Here we test the hypothesis that a time-resolved computational model of cortical sound representation enables the detailed reconstruction of individual real-life sound spectrograms (and corresponding waveforms) from fMRI measurements of auditory cortical response patterns. Our hypothesis builds upon results from computational modeling (Klein et al., 2003; Chi et al., 2005), animal electrophysiology (Kowalski et al., 1996; deCharms, 1998) and human functional neuroimaging (Santoro et al., 2014), which suggest that sound spectrograms are processed in the auditory cortex through frequency-localized neural filters tuned to specific combinations of spectral and temporal modulations.

\section{Results}

We applied a stimulus reconstruction approach to data from two fMRI experiments at 7T in humans (see (De Martino et al., 2013; Moerel et al., 2013) for Experiment 1 and Chapter 3 for Experiment 2). FMRI responses were recorded from the auditory cortex while subjects (Experiment 1: $n=5$; Experiment 2: $n=2$ ) listened to a large set of real-life sounds, including speech samples, music pieces, animal cries, scenes from nature, and tool sounds (see Materials and Methods). Reconstruction models were defined as linear decoders that used patterns of fMRI activation to predict features of a multi-dimensional stimulus representation, in which sounds were expressed according to their time-varying spectro-temporal modulation content (Chi et al., 2005) (Figure 4.1). In order to reduce dimensionality, the temporal dimension was downsampled to 10 time points (see Materials and Methods). The outputs of the reconstruction models were combined to obtain the spectrogram (and waveform) of the presented sounds from their predicted modulation representation.

Reconstruction accuracy in the modulation space

Using a subset of $\mathrm{fMRI}$ data (training), we estimated a reconstruction model for every feature of the modulation representation. We then assessed the ability of these models to accurately predict sounds' modulation content in a new, independent data set (testing), which involved sounds not used for model estimation. We quantified prediction accuracy as the average fraction of variance explained $\left(R^{2}\right)$ in the test sounds by the models' reconstruction (see Materials and Methods). The average $\mathrm{R}^{2}$ across test sounds was higher than 0 for all subjects and significantly higher than chance (Experiment 1: mean [SE] $=0.4$ [0.02], $p<0.001$, 
group level random effects 2-tailed paired t test; Experiment 2: mean [SE] $=0.38$ [0.02], $p<0.001$, single subject 2-tailed paired t test; Figure 4.2A).

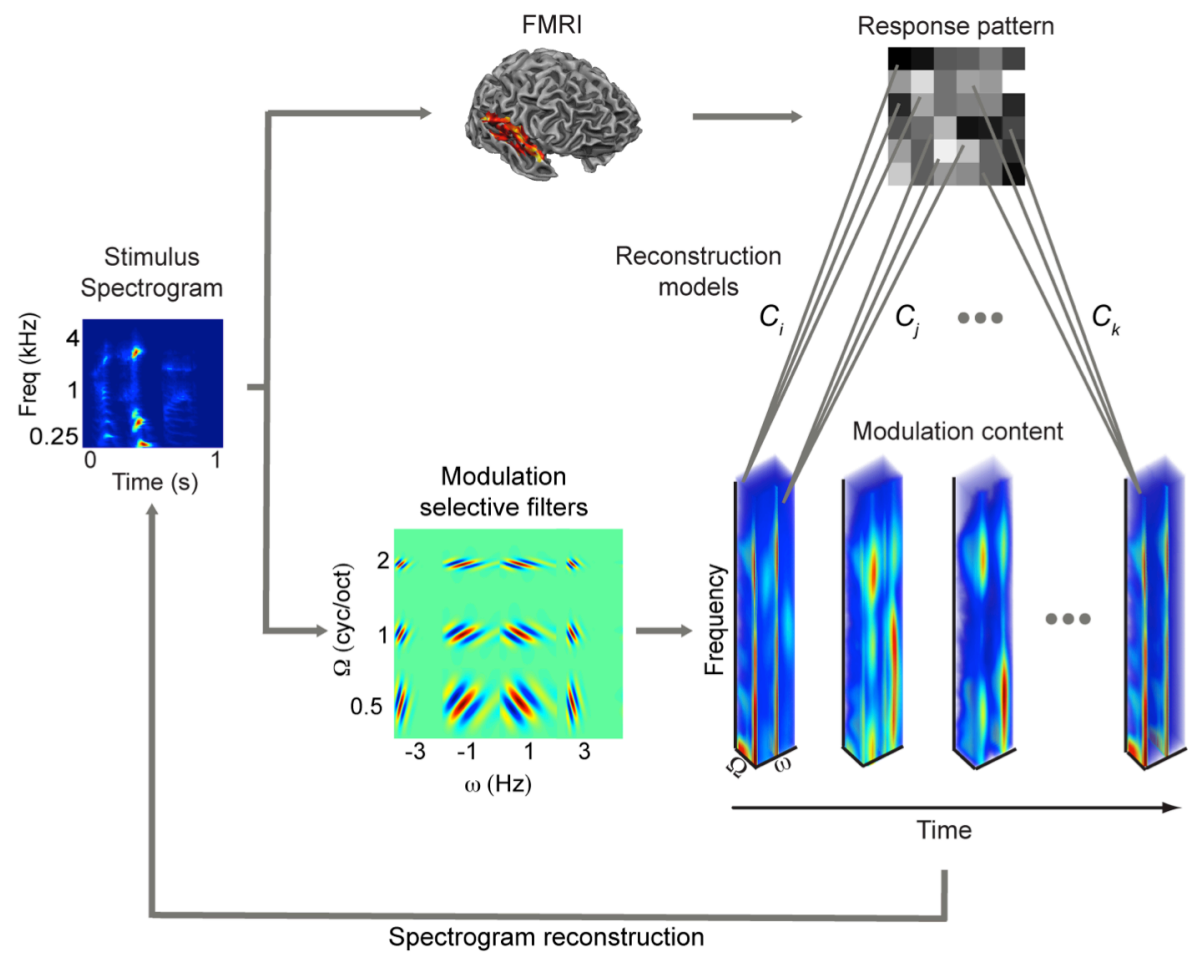

Figure 4.1. Schematic of reconstruction procedure. FMRI activity is measured while a natural sound is presented; reconstruction models use a linearly weighted sum of the response pattern to predict the modulation content of the stimulus. The weights $\left(C_{i}, C_{j}, \ldots, C_{k}\right)$ of the reconstruction models are determined using a separate training dataset. The stimulus representation in the modulation space is obtained through a bank of modulation selective filters tuned to different spectral modulations $(\Omega)$, temporal modulations ( $\omega)$, and direction (upwards/downwards). The output of the filter bank represents the time-varying spectro-temporal modulation content of the stimulus, that is the amount of energy that is present in the spectrogram at a given combination of $\Omega, \omega$, frequency $(f)$ and time (t). An independent reconstruction model is estimated for each point $(\Omega, \omega, f, t)$ of the modulation representation. Then, the outputs of the reconstructions models are combined to obtain the sound's spectrogram (and waveform) from the predicted modulation representation. 
A
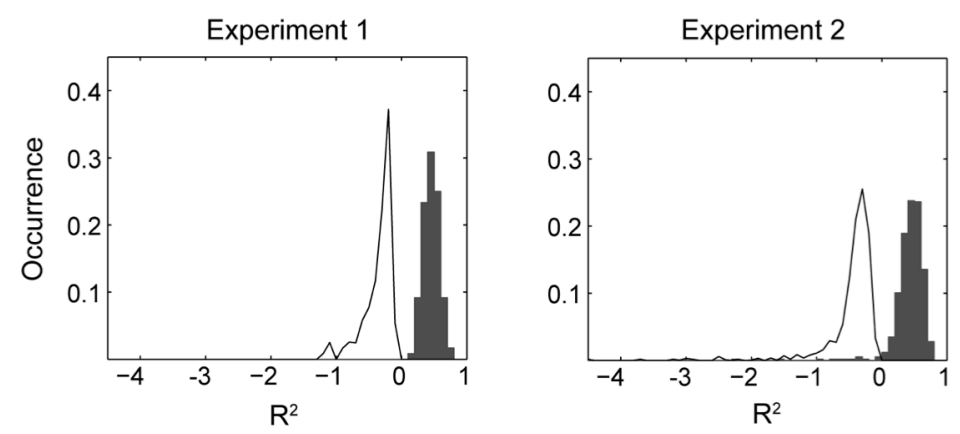

\section{B}
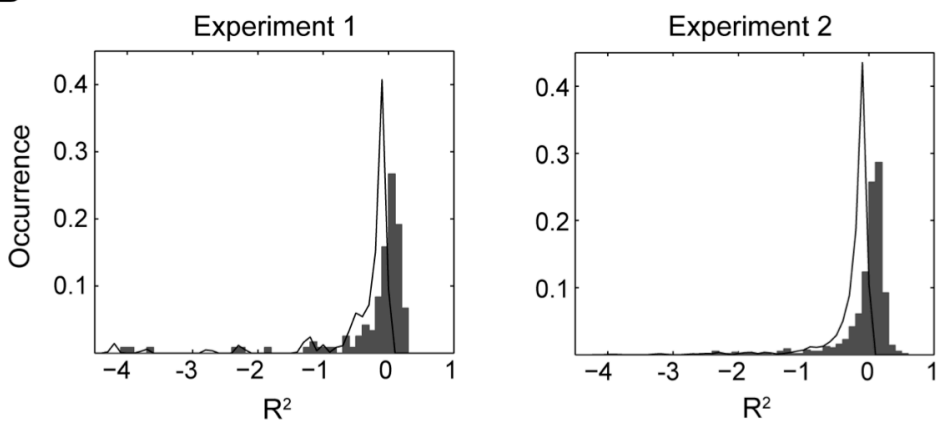

Figure 4.2. Overall reconstruction accuracy. (A) Distribution of $R^{2}$ across all test sounds and all subjects for the modulation representation, as estimated for Experiment 1 (left) and Experiment 2 (right). Grey bars represent the histogram of observed values; the black line outlines the empirical null distribution of $R^{2}$ (see Materials and Methods). (B) Distribution of $\mathrm{R}^{2}$ across all test sounds and all subjects for the time-frequency representation (Experiment 1: left; Experiment 2: right).

Identification accuracy in the modulation space

To evaluate the specificity of sound reconstruction, we performed a sound identification analysis (Kay et al., 2008b). Namely, we used the modulation representation predicted by the estimated models to identify which sound had been heard among all sounds in the test set. Each test sound was assigned with a score ranging between 0 and 1 and indicating the rank of the $R^{2}$ between sound's predicted and observed modulation content $(0$ indicates that the predicted modulation representation for a given stimulus was least similar to the observed one among all test stimuli; 1 indicates correct identification). The overall model's accuracy was obtained as the median score across all test sounds (see Materials and Methods). Identification accuracy was significantly higher than chance (0.5) for both datasets (Experiment 1: mean $[\mathrm{SE}]=0.79$ [0.03], group analysis: $p<0.05$, 
random effects 1-tailed Wilcoxon signed-rank test; single subjects analysis: 1-tailed $p<0.01$, permutation test; Experiment 2: mean [SE] $=0.79$ [0.04], single subjects analysis: 1 -tailed $p<0.01$, permutation test; Figure 4.3).

A

Experiment 1

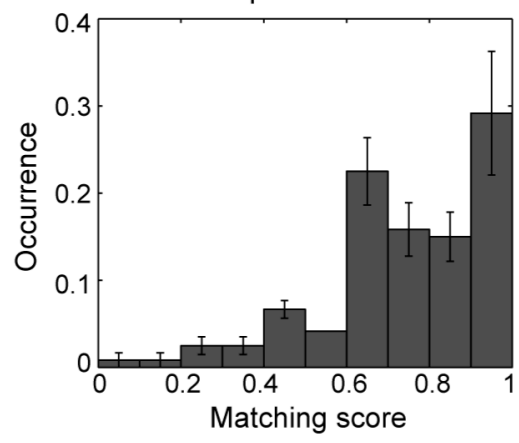

B

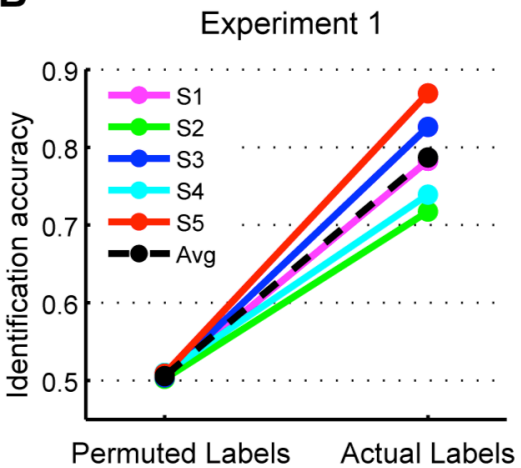

Experiment 2

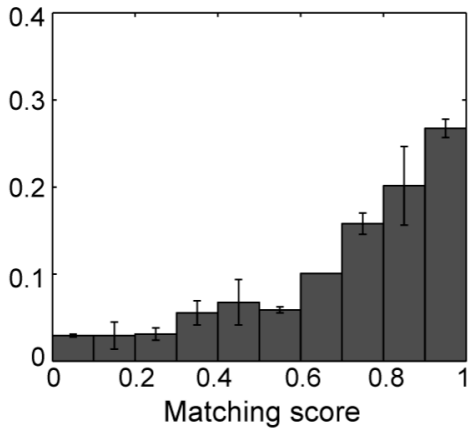

Experiment 2

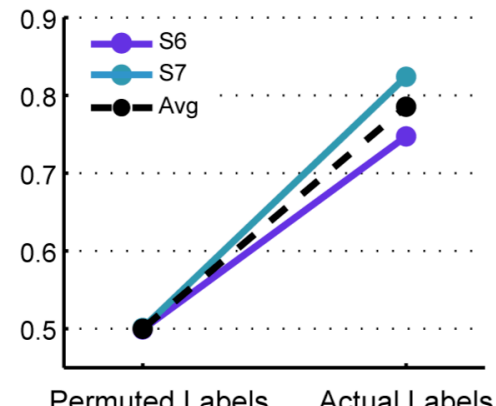

Figure 4.3. Identification performance of the modulation-based reconstruction model. (A) Average distribution of identification scores across subjects for Experiment 1 (left) and 2 (right) (mean $\pm \mathrm{SEM}, \mathrm{N}=5$ (2) for Experiment 1 (2)). (B) Identification accuracy for individual participants for Experiment 1 (left) and 2 (right). Each panel shows the accuracy obtained with correct labels and the accuracy derived by permuting the sound labels. Avg = Average.

\section{Control analysis}

As a control analysis, we trained a reconstruction model based on a timefrequency (i.e. spectrogram) representation of the stimuli that describes the timevarying energy content at each acoustic frequency (see Materials and Methods). 
The time-frequency based reconstruction model yielded $\mathrm{R}^{2}$ values close or lower than 0 (Experiment 1: median [IQR] $=-0.04$ [-0.22 0.05]; Experiment 2: median $[\mathrm{IQR}]=0.01[-0.110 .09])$, denoting poor prediction performance (Figure 4.2B). This indicates that the modulation representation is crucial for the decoding of temporal information from fMRI activity patterns.

Spectrogram and sound wave reconstruction from the modulation representation

The ultimate goal of a reconstruction decoder is sound re-synthesis. The restoration of the original sound wave from the reconstructed modulation representation is an engineering challenge that requires two steps of inverse operations: 1) from the modulation domain to the spectrogram, and 2) from the spectrogram to the waveform. The modulation representation is obtained via a complex wavelet transformation (see Materials and Methods and Chapter 1). When both magnitude and phase of the complex-valued output are preserved, the original sound spectrogram can be obtained by an inverse wavelet operation (Chi et al., 2005). However, our reconstruction model estimates only the magnitude information from $\mathrm{fMRI}$ activity patterns. Under these circumstances, an approximation of the spectrogram can be obtained by using iterative algorithms that attempt to retrieve the missing phase information (see Material and Methods). Likewise, the spectrogram itself is not perfectly invertible due to nonlinear operations involved in its calculation; therefore, an iterative procedure is necessary to restore an approximation of the sound wave from the spectrogram. When applied to actual sounds (i.e. not reconstructed from fMRI responses), these algorithms provide reconstructions that, although not perfect, are still intelligible (Chi et al., 2005).

For Experiment 1, we estimated spectrograms and sound waves of test sounds from the predicted modulation representation of subject S3 (data from this subject yielded the highest reconstruction quality). For Experiment 2, we reconstructed spectrograms and sound waves independently for each individual subject. Visual inspection of brain-based spectrogram reconstructions revealed that the spectral content of the sounds as well as prominent spectral, temporal and spectro-temporal modulations in the sounds could be faithfully recovered (see Figure 4.4 for examples of reconstructed spectrograms). Similarly, our re-synthesis algorithm produced sound waveforms that - in some cases - enabled a clear recognition of the original sound source (see e.g. Audio File S4.1). Although not intelligible, reconstructed waveforms of speech included audible and characteristic 
spectro-temporal modulations (see e.g. Audio File S4.2). To assess quantitatively the quality of our reconstructions, we performed an identification analysis of the spectrograms (see above and Materials and Methods). To account for errors related to iterative projection algorithms and temporal downsampling (see Materials and Methods), the reference spectrograms for the identification analysis were obtained by inverting the magnitude-only modulation representation of the original sounds (i.e. stimuli were projected into the modulation space and their magnitude-only modulation representation was downsampled to 10 time bins and back-projected to the time-frequency domain). For both experiments, spectrograms' identification accuracy was significantly higher than chance (Experiment 1: median $[\mathrm{IQR}]=0.65$ [0.33 0.91]; $\mathrm{p}<0.05,1$-tailed permutation test; Experiment 2: median $[\mathrm{IQR}]=0.66[0.350 .84]$ and $0.72[0.430 .89]$ for subjects S6 and $\mathrm{S} 7$ respectively; $\mathrm{p}<0.01,1$-tailed permutation test).

\section{Discussion}

Our results show that it is possible to reconstruct the spectrograms of real-life sounds from patterns of fMRI activity. An accurate reconstruction of the timeaveraged spectral components of sounds could be expected based on the tonotopic organization of the auditory cortex, which converts sound frequency information into spatial maps measurable with fMRI (Formisano et al., 2003; Da Costa et al., 2011; Moerel et al., 2012). Our present findings additionally suggest that - although smoothed - the dynamic spectro-temporal components of the spectrograms can be successfully retrieved from fMRI response patterns. This is surprising given the intrinsic low temporal resolution of $\mathrm{FMRI}$ and the coarse temporal sampling ( $T R=2.6 \mathrm{~s}$ ) of brain responses. How is that possible? We trained many ( $n=153600$ ) multivariate decoders (one for each model feature and for each time window) based on the same estimates of fMRI responses to the sounds (see Materials and Methods). Thus, successful reconstruction of test sounds suggests that the acoustic modulation content at specific combinations of spectral scales and temporal rates is consistently mapped into distinct and reproducible spatial patterns. Together with recent results obtained using natural movies (Nishimoto et al., 2011), our findings demonstrate the potential of using slow BOLD signals for studying the cortical coding of rapidly varying perceptual processes.

Note that our data-driven voxel-by-voxel estimation of the hemodynamic response function followed the standard assumption of considering each sound as 
Original Original back-projected Reconstructed
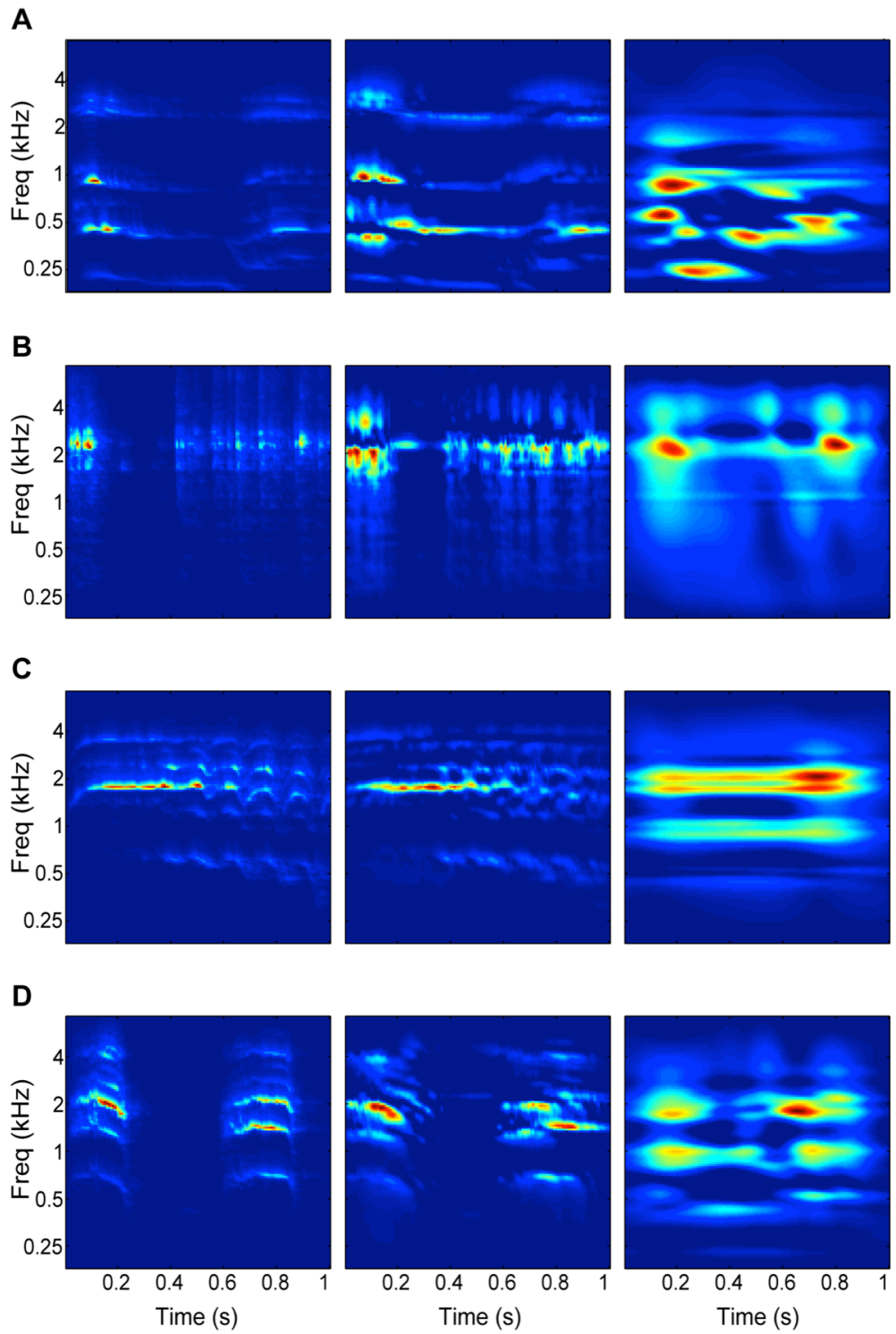

Figure 4.4. Sample reconstructed spectrograms. (A-D) Original and reconstructed spectrograms of four exemplary test sounds. Left: original spectrogram as extracted by the computational model mimicking early auditory processing. Middle: original spectrogram obtained by inverting the downsampled magnitude-only modulation representation computed by the auditory model. Note that the reconstructed spectrogram suffers from errors related to temporal downsampling and iterative projection algorithms (see Material and Methods). Right: sound spectrogram as reconstructed from the $\mathrm{fMRI}-$ based prediction of the modulation representation. 
a single neural event (see Materials and Methods). Further optimization of the hemodynamic response function for each separate feature (Nishimoto et al., 2011) could lead to further improvement of reconstruction performance.

The stimulus reconstruction algorithm implemented in the present study is very general and is not constrained to sampling from a predefined database (Miyawaki et al., 2008; Mesgarani et al., 2009; Nishimoto et al., 2011). It allows the reconstruction of an arbitrary sound solely based on sound-evoked cortical fMRI responses. Paisley et al. (2012) employed a similar computational approach to reconstruct the spectrogram of speech sounds from ECoG measurements. ECoG has an exquisite temporal resolution, which is not accessible with fMRI. However, the invasive nature of ECoG intrinsically limits the measurements to patients undergoing neuro-surgical treatments. Furthermore, recording grids in ECoG only sample neuronal responses from small brain regions, often limited to the cortical surface. Our study extends the possibility to investigate the cortical coding mechanisms for processing real-life sounds using fMRI. The large spatial coverage achievable with $\mathrm{fMRI}$ enables reconstructing sounds from responses of different auditory cortical areas. This can help unravel how a sound spectrogram is transformed through the network of auditory areas as well as shed light on mechanisms of areal specialization. Namely, the objective assessment of which stimulus features are accurately reconstructed in the distinct auditory areas may provide insights into their computational and functional role (see Chapter 3 ). The accessibility and non-invasiveness of fMRI makes it possible to address relevant questions e.g. on how the cortical representations of sound spectrograms change due to specific skill acquisitions (e.g. musical training), brain development and aging or hearing loss. Our methodological approach might have significant implications for the development of new auditory-based, fMRI-dependent communication systems for patients fully conscious and awake, yet unable to speak due severe motor paralysis. Similarly, studying the cortical representation of auditory hallucinations may be a relevant clinical application of our method. Auditory hallucinations are internally generated events that share the perceptual vividness of externally presented sounds. Primary auditory areas are activated during auditory hallucinations (Dierks et al., 1999); thus, our model-based analyses could be used for reconstructing the spectro-temporal structure of the hallucinations and "listening" to them. While certainly attractive, these applications require extensive developments and refinements of the currently proposed methods together with careful validation under covert but experimentally controlled conditions, such as mental auditory imagery in healthy subjects. 


\section{Materials and Methods}

\section{Experimental procedure}

Subjects, stimuli, experimental design, MRI parameters, and data preprocessing for Experiment 1 (De Martino et al., 2013; Moerel et al., 2013) and 2 have been described in Chapters 2 and 3, respectively. Briefly, fMRI responses were recorded from the auditory cortex while subjects (Experiment 1: $n=5$; Experiment 2: $n=2$ ) listened to recordings of a large sample of natural sounds (Experiment 1: 168 sounds, Experiment 2: 288 sounds; duration: 1 s, sampling frequency: $16 \mathrm{kHz}$ ). Stimuli included human vocal sounds (both speech and nonspeech, e.g., baby cry, laughter, coughing), animal cries (e.g., dog, cat, horse), musical instruments (e.g., piano, flute, drums), scenes from nature (e.g., rain, wind, thunder), and tool sounds (e.g., keys, scissors, vacuum cleaner). In Experiment 1, data were subdivided into six training runs and two testing runs. In the training runs, 144 of the 168 stimuli were presented with 3 repetitions overall (i.e. each sound was presented in 3 of the 6 training runs). The remaining 24 sounds were presented in the testing runs and repeated 3 times per run. In Experiment 2, stimuli were divided into 4 non-overlapping sets of 72 sounds each. We presented one stimulus set per run and every set was presented three times (i.e. 3 runs per set).

In both experiments, $\mathrm{fMRI}$ time series were acquired according to a fast event-related scheme (Experiment 1: TR $=2600 \mathrm{~ms}$; TA $=1200 \mathrm{~ms}$; TE $=30 \mathrm{~ms}$; GRAPPA acceleration X3; partial Fourier 6/8; voxel size $=1.5 \times 1.5 \times 1.5 \mathrm{~mm}^{3}$; silent gap $=1400 \mathrm{~ms} ;$ Experiment 2: TR $=2600 \mathrm{~ms} ; \mathrm{TA}=1200 \mathrm{~ms} ; \mathrm{TE}=19 \mathrm{~ms} ; \mathrm{GRAPPA}$ acceleration $\mathrm{X} 2$; partial Fourier $=6 / 8$; voxel size $=1.5 \times 1.5 \times 1.5 \mathrm{~mm}^{3}$; silent gap $=$ $1400 \mathrm{~ms})$. Within each run, stimuli were randomly spaced at a jittered inter-stimulus interval of 2, 3, or 4 TRs and presented, with additional random jitter, in the silent gap between acquisitions.

\section{Modulation representation}

The stimulus representation in the modulation space was obtained as the output of a biologically inspired model of auditory processing (Chi et al., 2005), which explicitly encodes the modulation content of a sound spectrogram. The auditory model consists of two main components: an early stage that accounts for the transformations that acoustic signals undergo in the early auditory system, from the cochlea to the midbrain; and a cortical stage that simulates the processing of the acoustic input at the level of the (primary) auditory cortex (see Chapter 1 for a 
detailed description of the model). We derived the auditory spectrogram and its modulation content using the "NSL Tools" package (available at http://www.isr.umd.edu/Labs/NSL/Software.htm) and customized Matlab code (The MathWorks Inc.). Sounds' spectrograms were generated using a bank of 128 overlapping bandpass filters with constant- $\mathrm{Q}\left(\mathrm{Q}_{1 \mathrm{dod} B}=3\right)$ mimicking the auditory periphery. Filters were equally spaced along a logarithmic frequency axis and covered a range of 5.3 oct ( $f=180-7040 \mathrm{~Hz}$ ). The output of the filter bank underwent band-pass filtering (hair cell stage), first-order derivative with respect to the frequency axis, half-wave rectification, and short-term temporal integration with time constant $\tau=8 \mathrm{~ms}$ (midbrain stage). The modulation content of the auditory spectrogram was computed through a bank of 2D modulation selective filters with joint selectivity for spectral and temporal modulations (see Joint frequency-specific MTF-based model, Chapter 2). Filters were tuned to 6 spectral modulation frequencies $(\Omega=[0.5,0.8,1.1,1.7,2.6,4] \mathrm{cyc} / \mathrm{oct})$, and 10 temporal modulation frequencies $(\omega=[1,1.5,2.1,3.1,4.5,6.6,9.7,14.1,20.6,30] \mathrm{Hz})$. For every combination of spectral and temporal modulation frequencies, we defined two filters, each responding either to upward $(\omega<0)$ or downward $(\omega>0)$ frequency sweeps. Accordingly, the total number of modulation selective filters was $6 * 2 * 10=$ 120. The output of the cortical filter bank is a complex-valued representation of the spectrogram envelope as a function of time, frequency, spectral and temporal modulations, and directionality. The magnitude of such representation yields a phase-invariant measure of modulation content. We obtained a reduced modulation representation by defining 10 non-overlapping time bins and averaging over time the modulation energy within each of these bins. This resulted in a representation with 6 spectral modulation frequencies $\times 20$ temporal modulation frequencies (10 upwards and 10 downwards) $\times 10$ time bins $\times 128$ frequencies $=153600$ features. The above processing steps were applied to all stimuli, resulting into an $[\mathrm{N} \times \mathrm{F}]$ feature matrix $\mathbf{S}$ of modulation energy, where $\mathrm{N}$ is the number of sounds, and $\mathrm{F}$ is the number of features in the reduced modulation representation.

\section{Time-frequency representation}

The stimulus representation in the time-frequency space was obtained using only the input stage of the auditory model. First, we computed the spectrogram at 128 logarithmically spaced frequency values $(f=180-7040 \mathrm{~Hz}$ ). Then, we defined 5 non-overlapping time bins and averaged over time the frequency content within each time bin. This resulted in a reduced time-frequency representation with 128 
frequencies $\times 5$ time bins $=640$ features. The above processing steps were applied to all stimuli, resulting into an $[N \times F]$ feature matrix $\mathbf{S}$ of time-frequency content, where $N$ is the number of sounds, and $F$ is the number of features.

\section{Estimation and evaluation of the reconstruction models}

In Experiment 1, the reconstruction models were trained on six training runs and tested on two testing runs (see above). In Experiment 2, we implemented a 4fold cross validation scheme across the 4 stimulus sets (i.e. 9 runs for training and 3 for testing). For model evaluation, the 4 test sets were concatenated to quantify the overall model's performance. Note that training and testing included different stimuli in both experiments.

\section{Estimation of $\mathrm{fMRI}$ responses to natural sounds}

We computed voxels' responses to natural sounds using customized Matlab code (The MathWorks Inc.). For each voxel $i$, the response vector $Y_{i}[(N \times 1), N=$ number of sounds] was obtained in two steps. First, a hemodynamic response function (HRF) common to all stimuli in the training runs was estimated via a deconvolution analysis in which all stimuli were treated as a single condition. Then, using this HRF and one predictor per sound, we computed the beta weight of each sound (Kay et al., 2008a). Beta weights were estimated separately for training and testing sounds. Further analyses were performed on voxels with a significant positive response to the training sounds $(p<0.05$, uncorrected in order not to be too stringent at this stage of the process) within an anatomically defined mask, which included HG, PT, PP, and STG.

\section{Estimation of model parameters}

We defined the stimulus reconstruction model as the linear relationship between patterns of fMRI activation and the stimulus space (modulation or timefrequency representation). Independent reconstruction models were trained for every feature in the stimulus representation (153600 for the modulation space and 640 for the time-frequency space), as follows. The stimulus feature $S_{i}\left[N_{\text {train }} \times 1\right]$ was modeled as a linear transformation of the multivoxel response pattern $\mathbf{Y}_{\text {train }}\left[N_{\text {train }} x\right.$ $V$ plus a bias term $b_{i}$ and a noise term $n\left[N_{\text {train }} x 1\right]$ as follows:

$$
S_{i}=\mathbf{Y}_{\text {train }} C_{i}+b_{i} 1+n
$$

where $N_{\text {train }}$ is the number of sounds in the training set, $V$ is the number of voxels, 1 
is a $\left[N_{\text {train }} \times 1\right]$ vector of ones, and $C_{i}$ is a $[V \times 1]$ vector of model parameters, whose elements $c_{i j}$ quantify the contribution of voxel $j$ to the encoding of feature $i$.

The solution to Equation 1 was computed using kernel ridge (Hoerl and Kennard, 1970; Bishop, 2006). The regularization parameter $\lambda$ was determined independently for each feature by generalized cross validation (Golub et al., 1979).

\section{Model evaluation: reconstruction accuracy}

Given the trained model $\tilde{\mathbf{C}}[V \times F]$, and the patterns of fMRI activity for the test sounds $\mathbf{Y}_{\text {test }}\left[N_{\text {test }} \times V\right]$, the predicted feature matrix $\hat{\mathbf{S}}_{\text {test }}\left[N_{\text {test }} \times F\right]$ for the test set was obtained as follows:

$$
\hat{\mathbf{S}}_{\text {test }}=\mathbf{Y}_{\text {test }} \tilde{\mathbf{C}}
$$

We quantified model's prediction accuracy as the fraction of variance explained $\left(R^{2}\right)$ in the test sounds by the model's reconstruction. For each stimulus $s$, we computed $\mathrm{R}^{2}$ as follows:

$$
R^{2}=1-\frac{\sum_{k=1}^{F}\left(s_{k}-\hat{s}_{k}\right)^{2}}{\sum_{k=1}^{F}\left(s_{k}-\bar{s}\right)^{2}}
$$

where $s_{k}$ is the k-th measured feature, $\hat{s}_{k}$ is the k-th predicted feature, and $\bar{S}$ is the mean of sound $s$ in the features space. $R^{2}$ values range from -Inf to 1 and represent a relative measure of how well the model predicts the data, with the reference model being the mean of the data itself.

For each stimulus in the test set, we obtained the empirical null-distribution of $\mathrm{R}^{2}$ by randomly permuting ( $P=500$ permutations) the feature labels of the reconstructed sound (i.e. $F$ in matrix $\mathbf{S}$ ) and computing $\mathrm{R}^{2}$ for each permutation. In order to prevent sample-related variability, the same permutations were applied to all sounds and all subjects.

Model evaluation: identification accuracy

Additionally to $\mathrm{R}^{2}$, we quantified model's prediction accuracy by performing a sound identification analysis (Kay et al., 2008b). Namely, we used sounds 
reconstructed from $\mathrm{fMRI}$ activity patterns to identify which sound had been heard among all sounds in the test set.

For each stimulus $s_{i}$, we computed the $\mathrm{R}^{2}$ between its predicted representation $\hat{S}_{\text {test }}\left(s_{i}\right)[1 \times \mathrm{F}]$ and all stimuli $S_{\text {test }}\left(s_{j}\right)[1 \times F], j=1,2, \ldots, N$. The rank of the $R^{2}$ between predicted and actual sound for stimulus $s_{i}$ was selected as a measure of the model's ability to correctly match $S_{\text {test }}\left(s_{i}\right)$ with its prediction $\wedge$ $S_{\text {test }}\left(s_{i}\right)$. The matching score $m$ for stimulus $s_{i}$ was obtained by normalizing the computed rank between 0 and 1 as follows $(m=1$ indicates correct match; $m=0$ indicates prediction for stimulus $s_{i}$ was least similar to the actual one among all stimuli):

$$
m\left(s_{i}\right)=1-\frac{\operatorname{rank}\left(s_{i}\right)-1}{N_{\text {test }}-1}
$$

Normalized ranks were computed for all stimuli in the test set, and the overall model's accuracy was obtained as the median of the matching scores across stimuli. To assess the statistical significance of the identification accuracy, we computed its null distribution by randomly permuting ( $P=200$ permutations) the test sounds (i.e. $N_{\text {test }}$ in $\hat{\mathbf{S}}_{\text {test }}$ ) and computing the identification accuracy for each permutation. We applied the same permutations to all subjects to prevent samplerelated variability.

Sound wave reconstruction from brain-based prediction

We used customized Matlab code (The MathWorks Inc.) to implement a direct projection algorithm for an approximate reconstruction of the auditory spectrogram from the fMRI-based prediction of the modulation representation. The algorithm can be summarized as follows (Chi et al., 2005):

1) Interpolate the magnitude representation to the original temporal resolution (magnitude information had been predicted at 10 temporal points, see above).

2) Initialize a random, non-negative auditory spectrogram.

3) Compute magnitude and phase of the modulation representation of the spectrogram.

4) Replace the magnitude from step 3 with the magnitude predicted from the fMRI activity patterns. 
5) Invert the complex-valued modulation representation to the spectrogram domain.

6) Half-wave rectify the spectrogram and repeat from step 3 until a predefined number of iterations is reached.

Finally, the sound waveform is obtained by inverting the estimated spectrogram. Because of the nonlinear operations in the early stage of the auditory model, also the reconstruction from the spectrogram is only an approximation. Here we used the iterative reconstruction algorithm (Chi et al., 2005) implemented in the "NSL Tools" package available at http://www.isr.umd.edu/Labs/NSL/Software.htm).

For Experiment 1, we applied the iterative procedure (10 iterations) to the predicted modulation representation of subject S3. To reduce noise in the reconstruction, we included only features whose predicted amplitude was above 0.2 in all subjects. For Experiment 2, the iterative reconstruction algorithm (10 iterations) was applied to each subject independently. A threshold of 0.2 was imposed on the predicted modulation representation of the subject under consideration. We performed an identification analysis to assess the degree of matching between reconstructed and observed spectrograms (see Model evaluation: identification accuracy). To account for errors related to temporal downsampling (to 10 time bins) and iterative projection algorithms, the reference spectrograms for the identification analysis were obtained by inverting the downsampled magnitude modulation representation of the original sounds (10 iterations). We computed the null distribution of the identification accuracy by randomly permuting ( $P=200$ permutations) the test sounds and computing the identification accuracy for each permutation.

\section{References}

Bialek W, Rieke F, de Ruyter van Steveninck RR, Warland D (1991) Reading a neural code. Sci $252: 1854-1857$.

Bishop C (2006) Pattern Recognition and Machine Learning. New York: Springer.

Chi T, Gao Y, Guyton MC, Ru P, Shamma S (1999) Spectro-temporal modulation transfer functions and speech intelligibility. J Acoust Soc Am 106:2719-2732. 
Chi T, Ru P, Shamma S (2005) Multiresolution spectrotemporal analysis of complex sounds. J Acoust Soc Am 118:887-906.

Da Costa S, van der Zwaag W, Marques JP, Frackowiak RSJ, Clarke S, Saenz M (2011) Human primary auditory cortex follows the shape of Heschl's gyrus. J Neurosci 31:14067-14075.

De Martino F, Moerel M, van de Moortele P-F, Ugurbil K, Goebel R, Yacoub E, Formisano $E$ (2013) Spatial organization of frequency preference and selectivity in the human inferior colliculus. Nat Commun 4:1386.

deCharms RC (1998) Optimizing Sound Features for Cortical Neurons. Science (80) 280:1439-1444.

Dierks T, Linden DEJ, Jandl M, Formisano E, Goebel R, Lanfermann H, Singer W (1999) Activation of Heschl's Gyrus during Auditory Hallucinations. Neuron 22:615-621.

Drullman R, Festen J, Plomp R (1994) Effect of temporal envelope smearing on speech reception. J Acoust Soc Am 95:1053-1064.

Elliott TM, Theunissen FE (2009) The modulation transfer function for speech intelligibility. PLoS Comput Biol 5:e1000302.

Formisano E, Kim DS, Di Salle F, van de Moortele PF, Ugurbil K, Goebel R (2003) Mirror-symmetric tonotopic maps in human primary auditory cortex. Neuron 40:859-869.

Golub G, Heath M, Wahba G (1979) Generalized Cross-Validation as a Method for Choosing a Good Ridge Parameter. Technometrics 21:215-223.

Hoerl AE, Kennard RW (1970) Ridge Regression: Biased Estimation for Nonorthogonal Problems. Technometrics 12:55-67.

Kay KN, David S V, Prenger RJ, Hansen KA, Gallant JL (2008a) Modeling lowfrequency fluctuation and hemodynamic response timecourse in eventrelated fMRI. Hum Brain Mapp 29:142-156.

Kay KN, Naselaris T, Prenger RJ, Gallant JL (2008b) Identifying natural images from human brain activity. Nature 452:352-355. 
Klein DJ, König P, Körding KP (2003) Sparse Spectrotemporal Coding of Sounds. EURASIP J Adv Signal Process 2003:659-667.

Kowalski N, Depireux DA, Shamma SA (1996) Analysis of dynamic spectra in ferret primary auditory cortex. II. Prediction of unit responses to arbitrary dynamic spectra. J Neurophysiol 76:3524-3534.

Mesgarani N, Chang EF (2012) Selective cortical representation of attended speaker in multi-talker speech perception. Nature:1-5.

Mesgarani N, David S V, Fritz JB, Shamma S a (2009) Influence of context and behavior on stimulus reconstruction from neural activity in primary auditory cortex. J Neurophysiol 102:3329-3339.

Miyawaki Y, Uchida H, Yamashita O, Sato M, Morito Y, Tanabe HC, Sadato N, Kamitani $Y$ (2008) Visual image reconstruction from human brain activity using a combination of multiscale local image decoders. Neuron 60:915-929.

Moerel M, De Martino F, Formisano E (2012) Processing of natural sounds in human auditory cortex: tonotopy, spectral tuning, and relation to voice sensitivity. J Neurosci 32:14205-14216.

Moerel M, De Martino F, Santoro R, Ugurbil K, Goebel R, Yacoub E, Formisano E (2013) Processing of natural sounds: characterization of multipeak spectral tuning in human auditory cortex. J Neurosci 33:11888-11898.

Naselaris T, Prenger RJ, Kay KN, Oliver M, Gallant JL (2009) Bayesian reconstruction of natural images from human brain activity. Neuron 63:902915.

Nishimoto S, Vu AT, Naselaris T, Benjamini Y, Yu B, Gallant JL (2011) Reconstructing Visual Experiences from Brain Activity Evoked by Natural Movies. Curr Biol:1641-1646.

Pasley BN, David S V, Mesgarani N, Flinker A, Shamma S, Crone NE, Knight RT, Chang EF (2012) Reconstructing Speech from Human Auditory Cortex Zatorre R, ed. PLoS Biol 10:e1001251.

Patil K, Pressnitzer D, Shamma S, Elhilali M (2012) Music in our ears: the biological bases of musical timbre perception. PLoS Comput Biol 8:e1002759. 
Santoro R, Moerel M, De Martino F, Goebel R, Ugurbil K, Yacoub E, Formisano E (2014) Encoding of Natural Sounds at Multiple Spectral and Temporal Resolutions in the Human Auditory Cortex. PLoS Comput Biol 10:e1003412.

Schoenmakers S, Barth M, Heskes T, van Gerven M (2013) Linear reconstruction of perceived images from human brain activity. Neuroimage 83:951-961.

Shannon R V, Zeng F-G, Kamath V, Wygonski J, Ekelid M (1995) Speech Recognition with Primarily Temporal Cues. Sci $270: 303-304$.

Singh NC, Theunissen FE (2003) Modulation spectra of natural sounds and ethological theories of auditory processing. J Acoust Soc Am 114:3394-3411.

Stanley GB, Li FF, Dan Y (1999) Reconstruction of natural scenes from ensemble responses in the lateral geniculate nucleus. J Neurosci 19:8036-8042.

Thirion B, Duchesnay E, Hubbard E, Dubois J, Poline J-B, Lebihan D, Dehaene S (2006) Inverse retinotopy: inferring the visual content of images from brain activation patterns. Neuroimage 33:1104-1116.

\section{Supporting Information}

Audio File S4.1. Example audio of reconstructed bird chirp. The original sound is followed by the brain-based reconstruction.

Audio File S4.2. Example audio of reconstructed speech. The original sound is followed by the brain-based reconstruction. 
Chapter 5

\section{Summary and Conclusions}




\section{Summary of the main findings}

The present thesis investigated the sensory representation of natural sounds in the human auditory cortex. Specifically, the studies presented in the previous chapters were motivated by two general research questions. First, which acoustic features define the representational space of natural sounds in the human auditory cortex? Second, how do representations change across auditory cortical regions?

The study presented in Chapter 2 compared competing computational models of sound representation. The tested models described the processing of three relevant properties of natural sounds: frequency, temporal modulations and spectral modulations. Results showed that a model that represents spectral and temporal modulations jointly and in a frequency-specific fashion provides the best account of $\mathrm{fMRI}$ responses to natural sounds. Besides providing insights into the representation of natural sounds in the human auditory cortex, this finding implies that caution should be taken when interpreting results based on stimuli that vary along one dimension alone, as an interaction occurs among frequency, spectral and temporal modulations. Based on feature preference maps, Chapter 2 suggested the presence of a large-scale spatial topography of spectral and temporal modulation tuning and that the functional specialization of auditory cortical fields can be partially accounted for by their modulation tuning.

The spatial variation of spectral and temporal response properties throughout the auditory cortex was further investigated in Chapter 3. The main goal of this chapter was to characterize the contribution of distinct macro-anatomical auditory cortical regions to the encoding of natural sounds. We addressed this question by assessing which acoustic features could be accurately reconstructed from patterns of $\mathrm{fMRI}$ responses to natural sounds. Results showed that auditory regions on the superior temporal plane encode a broader range of acoustic features compared to auditory regions along the superior temporal gyrus, where high frequencies and fast temporal modulations are less faithfully represented. The analysis of the acoustic discriminability of incoming sounds revealed that already in primary regions - and progressively more in surrounding non-primary regions - the observed spectral and temporal tuning increases the acoustic distance among speech/voice sounds, but not among exemplars of other categories of natural sounds. These results support the hypothesis that auditory cortical representational mechanisms - including those at an early hierarchical level - are optimized for a subset of natural sounds that are behaviorally most relevant, namely speech/voice for human listeners. 
Chapter 4 tackled the challenge - so far unexplored - of reconstructing arbitrary natural sounds based on patterns of fMRI responses. Results showed that a time-resolved computational model that represents sounds according to their time-varying spectro-temporal modulation content enables the detailed reconstruction of natural sound spectrograms (and waveforms) from fMRI cortical response patterns. These findings provide a direct proof of validity of the encoding hypothesis postulated in Chapter 2. Additionally, they open up the possibility to study covert auditory processing such as selective attention and imagery, which is pivotal to the development of fMRI-based communication systems and brain computer interfaces.

In conclusions, the main findings of this thesis provide insights on how natural sounds are encoded in human auditory cortex and the proposed methodological approach constitutes an advance in the way this question can be addressed in future studies.

\section{Model-based analysis of fMRI responses: Encoding vs Decoding}

In the present thesis we applied both encoding (Chapter 2) and decoding (Chapters 3 and 4) techniques. A discussion on general merits of decoding/encoding approaches can be found in Naselaris et al. (2010). Below we discuss their differences in relation to the specific research questions of the thesis.

Both encoding and decoding are well-suited for testing hypotheses of stimulus representation (Naselaris et al., 2010). Whereas encoding can be used to highlight tuning properties of individual voxels, decoding might be more appropriate when the goal is to characterize the representational content of a population of voxels or a cortical region (Mesgarani et al., 2009). In the encoding approach, a (multidimensional) tuning function is estimated for each voxel. This function represents the voxel's input-output relationship, i.e. the relative contribution of each stimulus feature to the voxel response. Due to its univariate (i.e. voxel-wise) nature, results from individual voxels must be integrated in order to obtain an input-output relationship for an entire cortical region. One approach is to generate cortical maps (i.e. spatial distributions) of the feature that elicits the highest response at each voxel (see Chapter 2). Conclusions based on this approach are inferred based on the assumption that stimulus features encoded with greater fidelity are those that maximally contribute to individual voxels responses and that are "preferred" by a larger number of voxels. However, the 
interpretation of tuning functions is still issue of debate - even at neuron's level and higher responses might not necessarily mean better encoding (Butts and Goldman, 2006). In summary, possible limitations of the encoding approach are the following: 1) tuning functions from individual voxels must be integrated into a population input-output relationship; 2) results are provided in terms of average activation levels (tuning functions), while conclusions must be drawn in the stimulus domain (i.e. which features are best encoded); therefore, knowledge of the link between activation levels and amount of stimulus information that is encoded is necessary.

These issues may be tackled with a decoding approach, which reconstructs stimulus features based on patterns of voxels' activity (Chapter 3 ). First, within the decoding framework, data from individual voxels are jointly modeled. This allows a multivariate characterization of a cortical region, without the need of integrating results derived from the modeling of individual voxels. Furthermore, the combined analysis of signals from multiple voxels may increase the sensitivity for stimulus information that may be represented in patterns of activity, rather than in individual voxels. Second, the amount of information that a given cortical region conveys about a set of stimulus features is made explicit in the accuracy with which those features can be reconstructed. Thus, as opposed to the encoding approach, there is no need to translate results obtained in the activation (or tuning function) space into conclusions drawn in the stimulus space.

\section{Future directions}

The work presented in this thesis provides the basis for future research aiming at unraveling the representation of natural sounds in the human auditory system. The present thesis focused on a sound representation that captures cortical tuning only along three stimulus dimensions (frequency, spectral modulations and temporal modulations). A challenge for future studies will be to extend the methodological framework presented here to explore increasingly complex models of sound representation.

A natural future direction for the work presented in this thesis is to investigate the temporal dynamics of spectral and temporal tuning properties, as well as their flexibility with respect to contextual changes, training, or task demands. The study presented in Chapter 3 revealed a cortical filtering mechanism that is designed to facilitate the acoustic discriminability of speech samples, arguably the natural sound with the highest behavioral relevance for human listeners. How are these 
mechanisms shaped by experience? Does the cortical tuning change as a function of the behavioral relevance that particular sound classes hold for specialized listeners (e.g. musicians)? Such long-term cortical reorganization mechanisms are only one aspect of functional plasticity. Electrophysiological studies in animals have shown that tasks' properties can influence neuronal tuning over short temporal scales (e.g. Fritz et al., 2003; David et al., 2012; Yin et al., 2014). Similarly, neuroimaging studies in humans have revealed that task-specific demands lead to different processing of the same physical stimulus (e.g. Alain et al., 2001; Bonte et al., 2014). However, previous reports have described this differential processing in terms of changes in localized activation strength (e.g. Alain et al., 2001) or distributed activation patterns (e.g. Bonte et al., 2014), leaving unanswered the question of which tuning mechanisms underlie these observations. The methodological framework presented in this thesis may help bridge this gap by estimating the feature tuning of auditory cortical regions under different behavioral conditions. This will provide a "task's fingerprint", that will allow us to hear sounds the way they are "heard" by the auditory cortex during the execution of a given task. From a technological perspective, the outcome of these studies may inform the design of engineering devices aiming at reproducing human auditory perceptual abilities.

Recent advances in MRI technology allow recording fMRI signals with unprecedented sub-millimeter resolution, opening the possibility to zoom into the laminar structure of the human auditory cortex (Koopmans et al., 2010, 2011; Polimeni et al., 2010). In combination with the methods described in the present thesis, these technological developments will allow studying the sensory representation of natural sounds across cortical layers.

\section{References}

Alain C, Arnott SR, Hevenor S, Graham S, Grady CL (2001) "What" and "where" in the human auditory system. Proc Natl Acad Sci U S A 98:12301-12306.

Bonte M, Hausfeld L, Scharke W, Valente G, Formisano E (2014) Task-dependent decoding of speaker and vowel identity from auditory cortical response patterns. J Neurosci 34:4548-4557. 


\section{Chapter 5}

Butts DA, Goldman MS (2006) Tuning Curves, Neuronal Variability, and Sensory Coding. PLoS Biol 4:e92.

David S V, Fritz JB, Shamma SA (2012) Task reward structure shapes rapid receptive field plasticity in auditory cortex. Proc Natl Acad Sci U S A 109:2144-2149.

Fritz J, Shamma S, Elhilali M, Klein D (2003) Rapid task-related plasticity of spectrotemporal receptive fields in primary auditory cortex. Nat Neurosci 6:1216-1223.

Koopmans PJ, Barth M, Norris DG (2010) Layer-specific BOLD activation in human V1. Hum Brain Mapp 31:1297-1304.

Koopmans PJ, Barth M, Orzada S, Norris DG (2011) Multi-echo fMRI of the cortical laminae in humans at 7 T. Neuroimage 56:1276-1285.

Mesgarani N, David S V, Fritz JB, Shamma S a (2009) Influence of context and behavior on stimulus reconstruction from neural activity in primary auditory cortex. J Neurophysiol 102:3329-3339.

Naselaris T, Kay KN, Nishimoto S, Gallant JL (2010) Encoding and decoding in fMRI. Neuroimage:1-11.

Polimeni JR, Fischl B, Greve DN, Wald LL (2010) Laminar analysis of 7T BOLD using an imposed spatial activation pattern in human V1. Neuroimage 52:13341346.

Yin P, Fritz JB, Shamma SA (2014) Rapid spectrotemporal plasticity in primary auditory cortex during behavior. J Neurosci 34:4396-4408. 


\section{Chapter 6}

\section{Knowledge valorization}




\section{Introduction}

In our everyday life we are constantly exposed to sounds from disparate sources. Our brain is able to use these sounds to accomplish remarkable tasks, such as recognizing somebody's voice, understanding speech, or enjoying music. The formidable nature of these tasks becomes evident when one considers the input signals that the auditory system must deal with, namely pressure waves with complex temporal patterns. To make sense of the myriad of sounds around us, the auditory system must transform these complex patterns into representations that facilitate the readout of behaviorally relevant information. The work presented in this thesis has provided new insights on how complex, real-life sounds are represented by the human auditory cortex. The following paragraphs will discuss potential social and economical implications of the outcomes of this thesis.

\section{Improvement of systems for automatic sound recognition}

Technology has become an integral part of our daily life and constant effort is being made towards the development of systems for automatic sound recognition, which can be employed in several practical areas, including:

- Speaker identification, i.e. recognizing a particular speaker by the characteristics of his voice (voice biometrics). Speaker identification can be used e.g. by security systems to verify the identity of a speaker and represents a quicker and more secure alternative to authentication through passwords, personal data or standardized questions.

- Speech recognition, i.e. the automatic identification of spoken words and their translation into text. The main goal of speech recognition algorithms is to allow reliable human-machine interaction via vocal communication. Although we are far from having a machine that is able to hold conversations with humans, current speech technology can be a useful tool for a variety of applications, including information retrieval (e.g. automated search in large audio-archives), dictation (e.g. note-taking systems), home appliance control, voice commands for smartphones.

- Music transcription, i.e. the process of reconstructing the notation of a piece of music from its recording.

- Environment monitoring: automatic sound recognition can be used for monitoring environments. For example, a home-monitor system could signal an intrusion by identifying the sound of windows being smashed. 
Despite their potential widespread application, current sound recognition systems are far from being perfect and their accuracy needs to be improved, especially in situations when interfering factors like e.g. background noise, sounds variability, or reverberation are present. The current effort for improvement is reflected in the attention that automatic sound recognition - and artificial intelligence in general - is receiving from software giants like e.g. Google and Facebook, which are striving to secure the world-leading experts in the field of deep learning, a branch of machine learning that promises to bring the mining of big data to a new level (Hinton et al., 2012). In March 2013, Google hired Geoffrey Hinton, a computer science professor at the University of Toronto and leading researcher in the deep learning field (University of Toronto, Media Room, 2013). Similarly, in January 2014, Google acquired DeepMind Technologies, an artificial intelligence start-up specialized in deep learning (Gibbs, 2014). These are just a few examples showing how active the hunting for machine learning talents is. However, powerful machine learning algorithms represent only one aspect of automatic sound recognition, namely the classification stage. In the processing pipeline of sound recognition systems, classification is preceded by feature extraction (Cowling \& Sitte, 2003), which represents an equally critical factor for the achievement of good recognition performance. The goal of feature extraction is to transform incoming sounds into a representation that highlights acoustic features relevant for classification. In other words, the feature extraction stage projects the input waveform into a feature space that is better suited for distinguishing between different sound classes. Numerous features spaces have been proposed and tested (e.g. Cowling \& Sitte, 2003; Kleinschmidt, 2002). However, despite the many decades of research, automatic sound recognition systems still lack robustness in adverse acoustic conditions. Human listeners, on the other hand, can effortlessly recognize sounds even in the presence of noise and despite the acoustic variability inherent in real-life sound sources. If the goal is to match human listeners performance, then building algorithms that mimic the human brain is likely to be the most effective strategy. In this framework, the work presented in this thesis can have a significant role in reducing the man-machine gap in sound recognition performance. Our studies have shed light on the feature space that the human auditory cortex uses to represent real-life sounds. This knowledge can readily be incorporated into state-of-the-art automatic sound recognition systems to improve their current performance.

Translating our results into a brain-inspired software for automatic sound recognition requires several steps. The first step should be the integration of our brain-based feature extraction software into the framework of current sound 


\section{Chapter 6}

recognition systems. This could be very much favored by collaborations with leading research groups in the field of automatic sound recognition. Commercialization opportunities could be generated by establishing contacts with companies potentially interested in our product. Among others, these may include Soundhound, Audio Analytic, Google, Facebook, Apple, Nuance Communications, Sejona R\&D, Microsoft, IBM.

\section{References}

Cowling, M., \& Sitte, R. (2003). Comparison of techniques for environmental sound recognition. Pattern Recognition Letters, 24(15), 2895-2907. doi:10.1016/S0167-8655(03)00147-8

Gibbs, S. (2014, January 27). Google buys UK artificial intelligence startup Deepmind for $f 400 \mathrm{~m}$. The Guardian. Retrieved from http://www.theguardian.com/technology/2014/jan/27/google-acquires-ukartificial-intelligence-startup-deepmind

Hinton, G., Deng, L., Yu, D., Mohamed, A., Jaitly, N., Senior, A., ... Kingsbury, B. (2012). Deep Neural Networks for Acoustic Modeling in Speech Recognition. IEEE Signal Processing Magazine, 29(6), 82-97.

Kleinschmidt, M. (2002). Robust speech recognition based on spectro-temporal processing. PhD Thesis, University of Oldenburg.

University of Toronto, Media Room. (2013). U of T neural networks start-up acquired by Google [Press release]. Retrieved from http://media.utoronto.ca/media-releases/u-of-t-neural-networks-start-upacquired-by-google/ 


\section{Acknowledgements}

Above all, I would like to express my gratitude to my supervisor, Elia. Working with you was a tremendous learning experience, both professionally and personally, and I feel honored that you gave me this opportunity. This thesis is the result of you teaching me how to do research and of us working side by side for long hours. I don't know many PhD students who can count on their supervisor taking the time to sit with them to analyze data or write papers and code. I am among the few lucky ones and I am very grateful for that. You taught me how to turn messy data into useful, sometimes exciting knowledge and how to look at results with critical eyes. Thank you very much for your contagious enthusiasm and optimism (how many times have I heard you say "e che ce vo'?!"?), and for enduring my stubborn skepticism and my constant strive for perfection. I have entered your office one million times saying "Elia, I am not convinced...", always confident that you would listen to me after a desperate "Ahhh Robbe', PER PIACERE!!!" :). Thank you for being always very straightforward with me and for showing a genuine interest in my future. Finally, thank you for understanding how essential it was for me to spend time in Lausanne and for granting me the flexibility to work from there so often.

Michelle, thank you for sharing your data with me. You are by far the most enthusiastic and dedicated scientist I know. I truly believe that I wouldn't have survived my first year without your patient support. You have taught me so much and your tenacity has been a great source of motivation throughout these years. You'll make a great PI one day!

Federico, I would literally not be writing this thesis if it wasn't for you. You were so thoughtful to make sure that I was absolutely certain of my decision of not entering the PhD program. I am very thankful for that. Thank you also for your critical and constructive feedback during our sparkling discussions, for your constant encouragement during these years and for your precious help with data collection.

I owe a big thank you to Giancarlo. You have been my savior whenever I had enough of brains and needed to go back to my engineering roots. I enjoyed very much our philosophical discussions about statistics, although keeping up with you was not always easy $(-)$. Thank you for your invaluable methodological support!

I am very grateful to the members of the auditory group that I had the pleasure to meet during my PhD: Elia, Milene, Lars R., Lars H., Michelle, Federico, Giancarlo, Anke, Kiki, Nick, Noel, Julien, Niels, Fren, Vittoria. Our meetings were always a 
great source of inspiration and an opportunity for me to learn and improve through your fruitful suggestions. Many thanks also to Agustin, for teaching me a lot about statistics and virtually taking me to Cuba with his stories. I would like to thank Essa Yacoub, for his prompt feedback on my manuscripts and his genuine appreciation of my work.

Riny, Christl, Annemie and Eva, thank you for your invaluable help with all the paperwork. Joost, Armin, and Judith, your support with BrainVoyager prevented me from smashing my laptop many times. My laptop and I deeply thank you for that.

Matteo and Tommaso, the men of my "weird Dutch family". I am actually getting emotional while writing these lines. Living together was so much fun and I feel really lucky that we were not just sharing a house, but living together as a family, as testified by our daily meetings to discuss the very serious question "what should we have for dinner?" (-). Thank you guys for all the fun times we shared, for your support during the darkest times, and for your effort to satisfy my inherently impossible request to buy me "una cosa sfiziosa" for dessert :).

A very big Grazie! goes to my good friend, colleague, office mate, temporary housemate Lars. I whish we lived close enough to have more of our movie nights, sushi dinners, or "psychotherapy sessions" while enjoying a cappuccino. I cannot thank you enough for helping me arrange my defense now that I am $800 \mathrm{~km}$ away from Maastricht.

I am very grateful to my friend and colleague Anke, for being always very supportive and straightforward with me. Your optimisms and cheerfulness made coming to work a lot more fun! I hope to see you and Sven in Switzerland some time soon!

Whenever in trouble, call Super Felix! I don't know how you do it, but you always manage to get to the core of a problem and make me see it from a completely new perspective! Thank you for sharing your honest and witty point of view about science, food, travels, life and much more.

Gesa, your reassuring presence has been precious in many occasions during these years, like the time I had to present at SfN, or the one that I freaked out on the plane back from Huston...thank you for letting me squeeze your hand and I am sorry if that hurt $:$.

Sergente a cavallo Anna :), I truly admire you for your determination and diligence. They have inspired me every day. Thanks for the nice chats we had at work and 
outside work, whenever my traveling schedule would match with your volleyball duties.

Franzi, you have been such a nice office mate and I whish we had met during a less busy period of my PhD. I would have loved to have more time to talk about our common passion for vintage furniture, crafting and sewing. Thank you Thomas for sharing with me your secrets on how to get good segmentations!

I would like to thank all the great people who make the $\mathrm{CN}$ department such a stimulating and enjoyable place to work. I feel lucky that I did my PhD in a place where learning something new was as easy as walking to the coffee corner, where interesting discussions were going on at all times. Alex, Joao, Martin, Matteo, Gojko, Marin, Britta, Marieke, Aline, Lars, Anke, Felix, Gesa, Anna, Michelle, thank you for all the nice dinners, parties and movie nights!

I would like to thank "Mr. Philips" for hiring my wise friend and "career advisor" Luca :). Thank you for always being there whenever I cannot think clearly. But above all, thank you for faking that fever and for making it possible for Giulio to travel back and forth from Lausanne to Maastricht. I owe you big times!

Many thanks to Vittoria, who joined our Italian household during my final, very stressful months in Maastricht. Thanks for feeding me (I loved your gnocchi!) and for taking care of the house while I was busy finishing my thesis.

I am very grateful to my supervisor in Geneva Narly, for being so patient with me being "almost finished" with my thesis for months. Many thanks also to my colleagues Alexis and Sanne for their support during these months.

Alla mia famiglia, mamma e papà, Annalisa, Clelia, Stefano, Lorenzo, Roberto, Alessandro, e quelli che arriveranno. Grazie! Mamma e papà, non sarei arrivata fin qua se non mi aveste insegnato ad essere tenace e a lavorare sodo per raggiungere i miei obiettivi. Grazie per avermi dato l'opportunità di seguire la mia curiosità, anche quando questa mi ha portato a $2000 \mathrm{~km}$ da casa. Isa, grazie per essere stata la mia compagna di viaggio sin dall'inizio. La mia avventura a Maastricht è iniziata con te che dormivi sul pavimento (:) ed è proseguita con i nostri fantastici viaggi pre- o post-conferenza. La prossima volta che andiamo a New York lo prendiamo quel traghetto! Clelia, hai capito finalmente in cosa consiste il mio lavoro (:)?! Grazie per essere sempre (fin troppo) schietta. Ogni tanto non c'è niente di più salutare di un reality check. Stefano, grazie per I'interesse genuino che hai sempre mostrato nel mio lavoro e nei miei progetti per il futuro. Lorenzo, nonostante tu abbia cercato di ammazzarmi sugli sci, ti sono 
grata per la sana calma serafica e imbranataggine con cui farcisci le nostre riunioni familiari (le tovaglie bianche e le piante di mamma ti sono un po' meno grate (:)). Roberto e Alessandro, i miei antidepressivi naturali. Grazie per essere stati così comprensivi con la zia che "studia sempre" (cito Alessandro ()). Spero che continuiate a guardare il mondo con la meraviglia e la curiosità che avete oggi.

Angela, grazie per avermi insegnato che perdersi d'animo non fa scomparire i problemi.

Giulio, my very personal "Accademia della Crusca". You have always been the biggest fan of my work and your compulsive curiosity makes me constantly discover new aspects of my projects. Thank you for always being so confident that I can achieve a goal, no matter how impossible it seems. But above all, thank you for taking me on the Ferris wheel...there is an amazing view from up here (:). 


\section{Publications}

\section{Articles}

*Authors share equal contribution

Moerel M, De Martino F, Santoro R, Yacoub E, Formisano E (2015) Representation of pitch chroma by multi-peak spectral tuning in human auditory cortex. Neuroimage 106:161-9

Santoro R, Moerel M, De Martino F, Goebel R, Ugurbil K, Yacoub E, Formisano E (2014) Encoding of Natural Sounds at Multiple Spectral and Temporal Resolutions in the Human Auditory Cortex. PLoS Comput Biol 10 (1): e1003412.

Moerel M, De Martino F, Santoro R, Ugurbil K, Goebel R, Yacoub E, Formisano E (2013) Processing of natural sounds: characterization of multipeak spectral tuning in human auditory cortex. J Neurosci 33:11888-11898

Santoro R*, Moerel M, De Martino F, Valente G, Ugurbil K, Yacoub E, Formisano $E^{*}$ (submitted) Re-synthesis of real-life sounds from non-invasively measured auditory cortical response patterns.

Santoro R, Moerel M, De Martino F, Valente G, Ugurbil K, Yacoub E, Formisano E (in preparation) Spectral and temporal properties of auditory cortical regions and their relation to the acoustic discriminability of natural sounds.

\section{Conference abstracts and proceedings}

Santoro R, Moerel M, De Martino F, Valente G, Ugurbil K, Yacoub E, Formisano E (2014) Brain listening: re-synthesis of natural sounds from fMRI auditory cortical response patterns. 9th FENS Forum of Neuroscience, Milan, Italy

Santoro R, Moerel M, De Martino F, Valente G, Ugurbil K, Yacoub E, Formisano E (2014) Brain Listening: Natural sounds re-synthesis from fMRI auditory cortical response patterns. 20th Annual Meeting of the Organization for Human Brain Mapping, Hamburg, Germany 
Moerel M, De Martino F, Santoro R, Ugurbil K, Yacoub E, and Formisano E (2014) Encoding of sound frequency and location in human subcortical structures. Joint Annual Meeting ISMRM-ESMRMB, Milan, Italy

Moerel M, De Martino F, Santoro R, Yacoub E, Formisano E (2013). Encoding of sound frequency and location in human subcortical structures: Preliminary results using functional MRI at 7T. Society for Neuroscience, New Orleans, San Diego, CA

Moerel M, De Martino F, Santoro R, Yacoub E, Formisano E (2013). Encoding of sound frequency and location in human subcortical structures: Preliminary results using functional MRI at 7T. The Tucker-Davis Technologies (TDT) Symposium on Advances and Perspectives in Auditory Neurophysiology, San Diego, CA

Santoro R, Moerel M, De Martino F, Ugurbil K, Yacoub E, Formisano E (2012). Representation of natural sounds in the human auditory cortex. Society for Neuroscience, New Orleans, LA. Selected talk.

Santoro R, Moerel M, De Martino F, Ugurbil K, Yacoub E, Formisano E (2012). Probing computational models of auditory cortical processing with natural sounds and functional MRI (3 and 7 Tesla). 4th International Conference on Auditory Cortex, Lausanne, Switzerland

Hausfeld L, Santoro R, Valente G, Formisano E (2012). Classification and Visualization of Multiclass fMRI Data Using Supervised Self-Organizing Maps. In: International Workshop on Pattern Recognition in Neurolmaging (PRNI), London, UK, $65-68$.

Moerel M, De Martino F, Santoro R, Ugurbil K, Yacoub E, Formisano E (2012). Functional networks in auditory cortex defined by ICA of neuronal population spectral tuning. 18th Annual Meeting of the Organization for Human Brain Mapping, Beijing, China

Moerel M, De Martino F, Santoro R, Ugurbil K, Yacoub E, Formisano E (2012). Octave-based spectral tuning in human auditory cortex. 4th International Conference on Auditory Cortex, Lausanne, Switzerland 
Santoro R, Moerel M, De Martino F, Formisano E (2011). Cortical Processing of Natural Sounds at Multiple Spectral Resolutions. 17th Annual Meeting of the Organization for Human Brain Mapping, Quebec City, Canada 


\section{Curriculum Vitae}

Roberta Santoro was born in Nocera Inferiore (Italy) on March 26 ${ }^{\text {th }} 1984$. In 2003 she completed her high school education at the "Liceo Scientifico Leonardo Da Vinci" in Vallo della Lucania. In the same year, she enrolled at the Faculty of Biomedical Engineering at Pisa University, where she obtained her Bachelor's degree (cum laude) in 2006, and her Master's degree (cum laude) in 2009. During the last year of her Master program, she spent one semester at the Maastricht Brain Imaging Center (Maastricht University), where she completed her Master thesis "Application of magnetoencephalography and spectro-temporal analysis methods to the study of real-life auditory scenes perception", under the supervision of Prof. Elia Formisano. In 2009, she started her Ph.D. program at the Department of Cognitive Neuroscience, Faculty of Psychology and Neuroscience, Maastricht University. She conducted her research under the supervision of Prof. Elia Formisano and co-supervision of Dr. Michelle Moerel and Dr. Federico De Martino. In March 2014 she joined the Brain and Language Lab at the University of Geneva, where she is currently working as post-doctoral fellow under the supervision of Prof. Narly Golestani. 